\title{
Generation of Synthetic Equivalents of Benzdiynes
}

\section{from Benzobisoxadisiloles}

Ya-Li Chen, Jiang-Qin Sun, Xin Wei, Wai-Yeung Wong and Albert W. M. Lee*

Department of Chemistry and Centre for Advanced Luminescence Materials,

Hong Kong Baptist University, Kowlooon Tong, Hong Kong, China

alee@hkbu.edu.hk

Supporting Information

\section{Contents}

General Information $\quad$ S3

FIGURE 1. X-ray Structure of $14 \quad$ S4

FIGURE 2. X-ray Structures of 19a, 19d, 20b, 20c and $22 \quad$ S4

FIGURE 3. X-ray Structure of 25a (syn) S4

FIGURE 4. X-ray Structure of 34b $\quad$ S4

${ }^{1} \mathrm{H}$ NMR spectrum of $\mathbf{1 2 b} \quad$ S5

${ }^{13} \mathrm{C}$ NMR spectrum of 12b $\quad$ S6

$\begin{array}{ll}{ }^{1} \mathrm{H} \text { NMR spectrum of } \mathbf{1 3} & \mathrm{S} 7\end{array}$

${ }^{13} \mathrm{C}$ NMR spectrum of $\mathbf{1 3} \quad$ S8

${ }^{1} \mathrm{H}$ NMR spectrum of $\mathbf{1 4} \quad$ S9

${ }^{13} \mathrm{C}$ NMR spectrum of $\mathbf{1 4} \quad \mathrm{S} 10$

${ }^{1} \mathrm{H}$ NMR spectrum of $\mathbf{1 5} \quad \mathrm{S} 11$

${ }^{13} \mathrm{C}$ NMR spectrum of $\mathbf{1 5} \quad \mathrm{S} 12$

${ }^{1} \mathrm{H}$ NMR spectrum of $\mathbf{1 6} \quad \mathrm{S} 13$

${ }^{13} \mathrm{C}$ NMR spectrum of $\mathbf{1 6} \quad \mathrm{S} 14$

${ }^{1} \mathrm{H}$ NMR spectrum of 19a $\quad \mathrm{S} 15$

${ }^{13}$ C NMR spectrum of 19a $\quad$ S16

$\begin{array}{ll}{ }^{1} \mathrm{H} \text { NMR spectrum of 19b } & \mathrm{S} 17\end{array}$

${ }^{13}$ C NMR spectrum of 19b $\quad$ S18

$\begin{array}{ll}{ }^{1} \mathrm{H} \text { NMR spectrum of 19c } & \text { S19 }\end{array}$

$\begin{array}{ll}{ }^{13} \mathrm{C} \text { NMR spectrum of 19c } & \text { S20 }\end{array}$

$\begin{array}{ll}{ }^{1} \mathrm{H} \text { NMR spectrum of 19d } & \text { S21 }\end{array}$

${ }^{13}$ C NMR spectrum of 19d $\quad$ S22 
${ }^{1} \mathrm{H}$ NMR spectrum of 19e $\quad$ S23

$\begin{array}{ll}{ }^{13} \mathrm{C} \text { NMR spectrum of 19e } & \text { S24 }\end{array}$

${ }^{1} \mathrm{H}$ NMR spectrum of 20a $\quad$ S25

${ }^{13} \mathrm{C}$ NMR spectrum of 20a $\quad$ S26

$\begin{array}{ll}{ }^{1} \mathrm{H} \text { NMR spectrum of 20b } & \text { S27 }\end{array}$

$\begin{array}{ll}{ }^{13} \mathrm{C} \text { NMR spectrum of 20b } & \text { S28 }\end{array}$

$\begin{array}{ll}{ }^{1} \mathrm{H} \text { NMR spectrum of 20c } & \text { S29 }\end{array}$

$\begin{array}{ll}{ }^{13} \mathrm{C} \text { NMR spectrum of 20c } & \text { S30 }\end{array}$

${ }^{1} \mathrm{H}$ NMR spectrum of 20d $\quad$ S31

${ }^{13} \mathrm{C}$ NMR spectrum of 20d $\quad$ S32

${ }^{1} \mathrm{H}$ NMR spectrum of 20e $\quad$ S33

$\begin{array}{ll}{ }^{13} \mathrm{C} \text { NMR spectrum of 20e } & \mathrm{S} 34\end{array}$

${ }^{1} \mathrm{H}$ NMR spectrum of 21

${ }^{13} \mathrm{C}$ NMR spectrum of $\mathbf{2 1} \quad \mathrm{S} 36$

$\begin{array}{ll}{ }^{1} \mathrm{H} \text { NMR spectrum of } 22 & \text { S37 }\end{array}$

$\begin{array}{ll}{ }^{13} \mathrm{C} \text { NMR spectrum of } 22 & \text { S38 }\end{array}$

${ }^{1} \mathrm{H}$ NMR spectrum of 25a/26a $\quad$ S39

${ }^{13} \mathrm{C}$ NMR spectrum of 25a/26a $\quad \mathrm{S} 40$

${ }^{1} \mathrm{H}$ NMR spectrum of $\mathbf{2 5 b / 2 6 b} \quad \mathrm{S} 41$

${ }^{13} \mathrm{C}$ NMR spectrum of 25b/26b $\quad \mathrm{S} 42$

${ }^{1} \mathrm{H}$ NMR spectrum of $\mathbf{2 7} \quad \mathrm{S} 43$

${ }^{13} \mathrm{C}$ NMR spectrum of $27 \quad \mathrm{~S} 44$

${ }^{1} \mathrm{H}$ NMR spectrum of $\mathbf{2 8} \quad \mathrm{S} 45$

${ }^{13} \mathrm{C}$ NMR spectrum of $\mathbf{2 8} \quad \mathrm{S} 46$

${ }^{1} \mathrm{H}$ NMR spectrum of $\mathbf{3 1}$

$\begin{array}{ll}{ }^{13} \mathrm{C} \text { NMR spectrum of } \mathbf{3 1} & \mathrm{S} 48\end{array}$

${ }^{1} \mathrm{H}$ NMR spectrum of 33a $\quad$ S49

${ }^{13} \mathrm{C}$ NMR spectrum of 33a $\quad$ S50

${ }^{1} \mathrm{H}$ NMR spectrum of 33b $\quad$ S51

${ }^{13} \mathrm{C}$ NMR spectrum of 33b $\quad \mathrm{S} 52$

${ }^{1} \mathrm{H}$ NMR spectrum of 33c $\quad$ S53

${ }^{13}$ C NMR spectrum of 33c $\quad$ S54

${ }^{1} \mathrm{H}$ NMR spectrum of 34b

${ }^{13} \mathrm{C}$ NMR spectrum of 34b $\quad$ S56

$\begin{array}{ll}{ }^{1} \mathrm{H} \text { NMR spectrum of 35b } & \text { S57 }\end{array}$

${ }^{13} \mathrm{C}$ NMR spectrum of $\mathbf{3 5 b} \quad \mathrm{S} 58$

${ }^{1} \mathrm{H}$ NMR spectrum of 35c $\quad$ S59

${ }^{13} \mathrm{C}$ NMR spectrum of 35c $\quad \mathrm{S} 60$ 
${ }^{1} \mathrm{H}$ NMR spectrum of $\mathbf{3 7 b} / \mathbf{3 8 b} \quad$ S61

${ }^{13} \mathrm{C}$ NMR spectrum of $\mathbf{3 7 b} / \mathbf{3 8 b} \quad \mathrm{S} 62$

General. NMR spectra were recorded on a Varian INOVA-400FT NMR spectrometer (400 MHz for ${ }^{1} \mathrm{H}$ and $100.6 \mathrm{MHz}$ for ${ }^{13} \mathrm{C}$ ) or a JEOL EX270 NMR spectrometer $\left(270 \mathrm{MHz}\right.$ for ${ }^{1} \mathrm{H}$ and $67.8 \mathrm{MHz}$ for $\left.{ }^{13} \mathrm{C}\right)$ with $\mathrm{CDCl}_{3}$ as the solvent. Chemical shifts are reported as parts per million (ppm) in $\delta$ unit in the scale relative to the resonance of $\mathrm{CDCl}_{3}\left(7.26 \mathrm{ppm}\right.$ in the ${ }^{1} \mathrm{H}, 77.00 \mathrm{ppm}$ for the central line of the triplet in the ${ }^{13} \mathrm{C}$ modes, respectively). Coupling constants $(J)$ are reported in Hz. IR spectra were determined on a Nicolet Magna-IR 550 spectrometer, date were given in $\mathrm{cm}^{-1}$. Low resolution mass spectra (LRMS) were obtained on a Finnigan MAT SSQ-710 spectrometer in FAB (positive ion) mode or E.I. mode and reported as $m / z$. High resolution mass spectra (HRMS) was recorded on a Applied Biosystem QSTAR Pulsar/LC/MS/MS, ESI-QTOF instrument. Melting points are uncorrected. Anhydrous magnesium sulfate was the drying agent throughout, and the silica gel for chromatography was 230-400 mesh. Element analyses were performed at the Shanghai Institute of Organic Chemistry, Chinese Academy of Science and Shanxi University. X-ray crystallographic data were collected with a Bruker AXS SMART 1000 CCD area-detector diffractometer with graphite-monochromated Mo-K $\alpha$ radiation $(\lambda=0.71073 \AA)$ at $293 \mathrm{~K}$. The collected frames were processed with proprietary software SAINT and an absorption correction was applied (SADABS) to the collected reflections. The structures of these molecules were solved by direct methods and expanded by standard difference Fourier syntheses using the software SHELXTL. 
FIGURE 1. X-ray Structure of 14

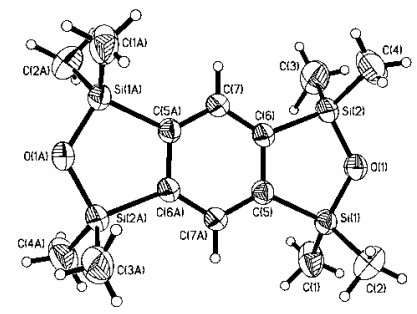

FIGURE 2. X-ray Structures of 19a, 19d, 20b, 20c and 22

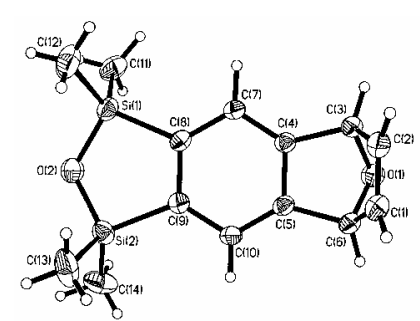

19a

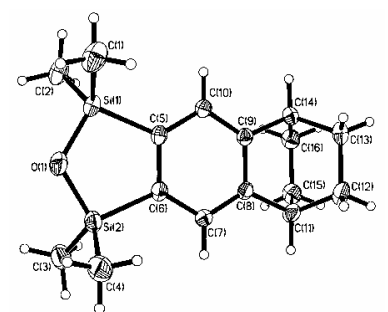

20c

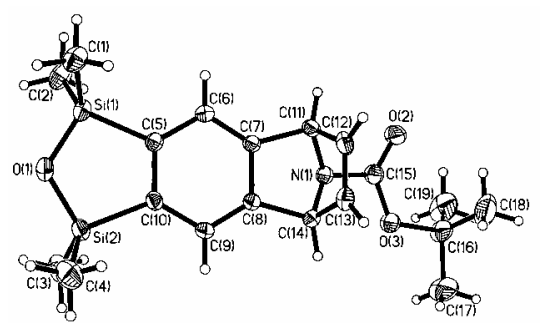

19d

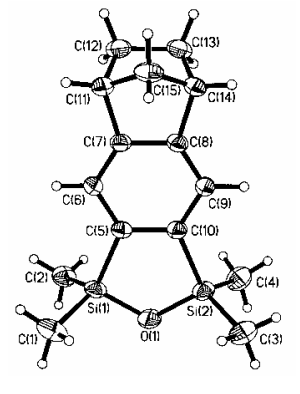

20b

FIGURE 3. X-ray Structure of 25a (syn)

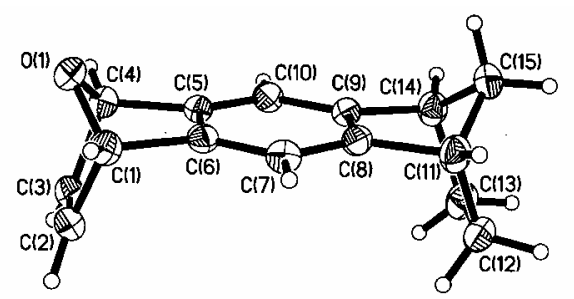

FIGURE 4. X-ray Structure of 34b

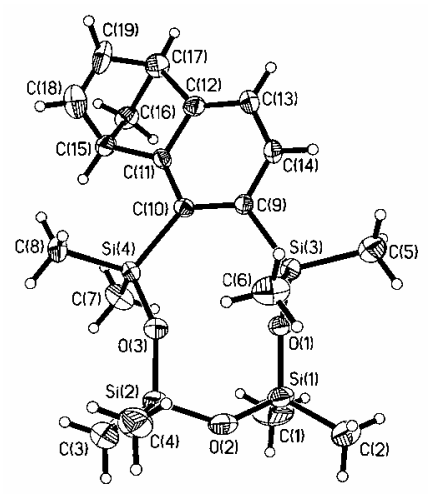


Ablert W. M. Lee et al. - Supporting Information

${ }^{1} \mathrm{H}$ NMR spectrum of $\mathbf{1 2 b}$

$500 \cdot 0-$

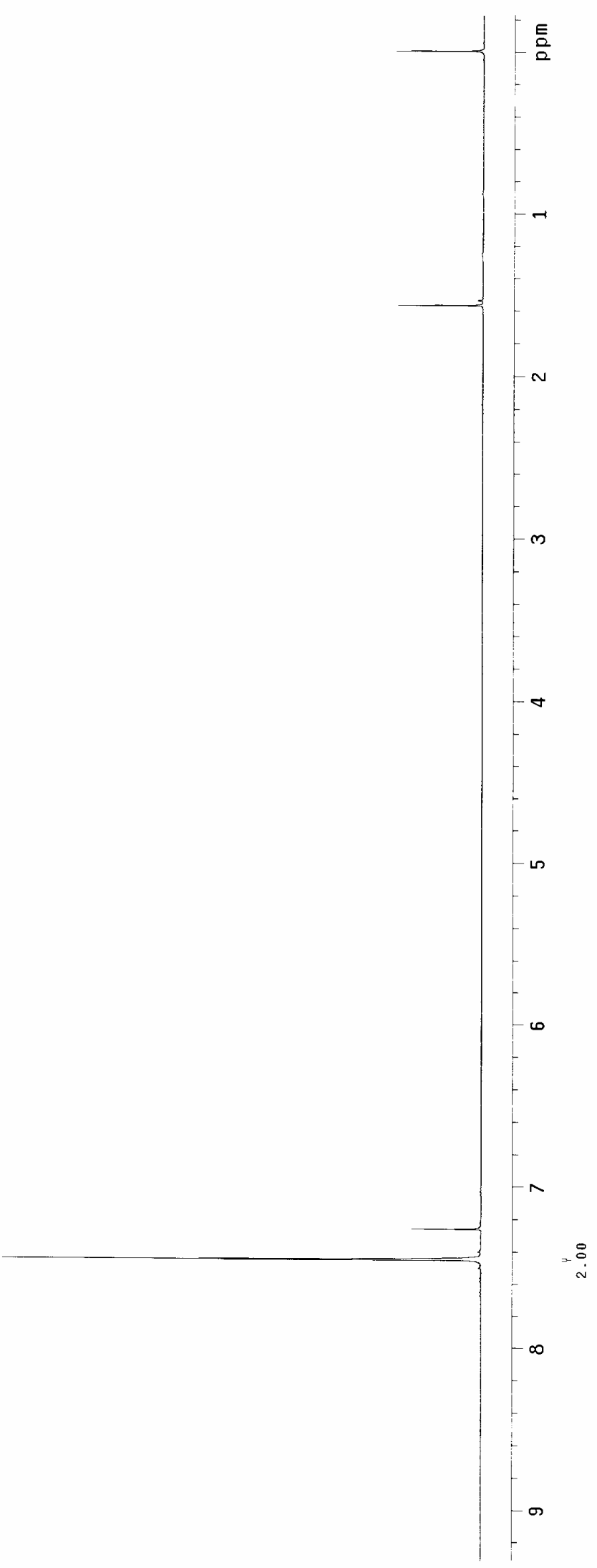

$092 \cdot 2$

ISt $\cdot 2$

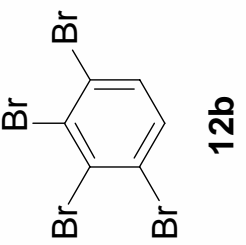


Ablert W. M. Lee et al. - Supporting Information

${ }^{13} \mathrm{C}$ NMR spectrum of $\mathbf{1 2 b}$

$089^{\circ} 92$

$000^{\circ} \angle L$

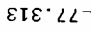

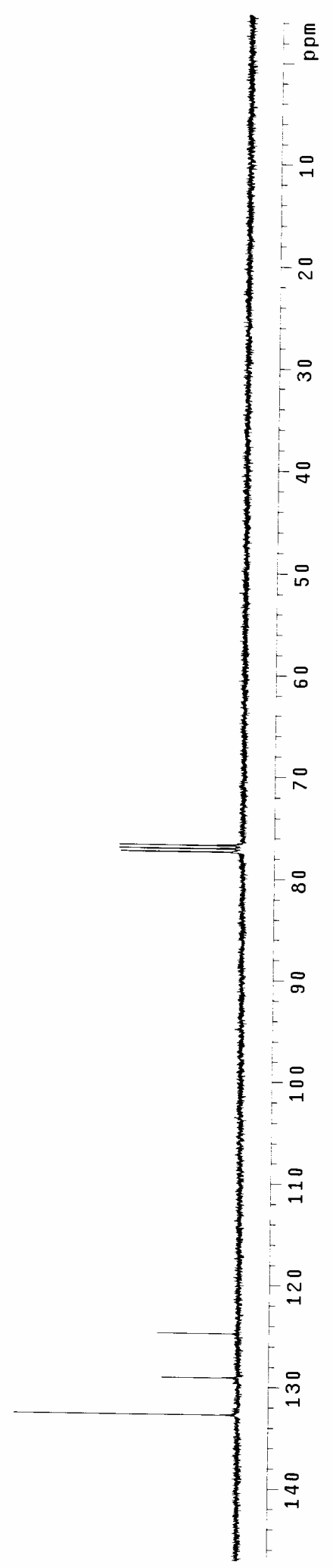


Ablert W. M. Lee et al. - Supporting Information

${ }^{1} \mathrm{H}$ NMR spectrum of $\mathbf{1 3}$
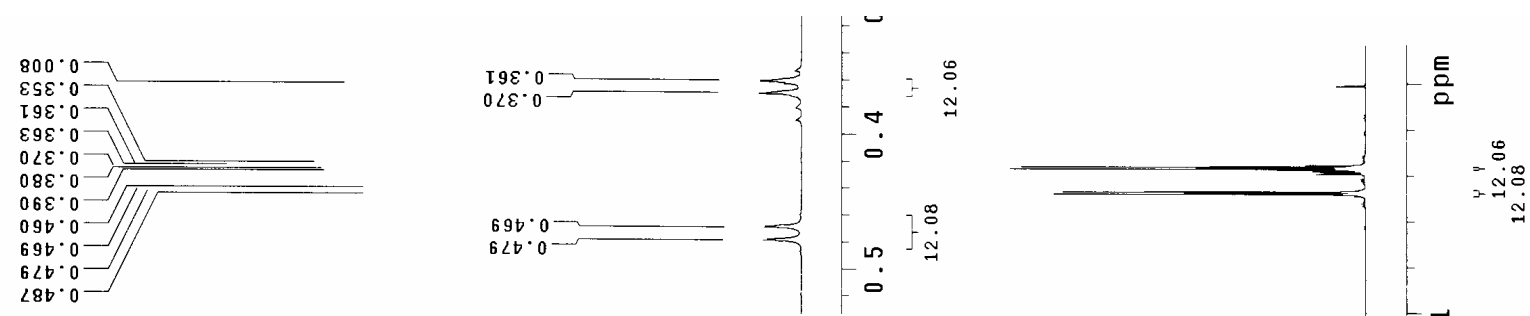

$89 \mathrm{~s} \cdot \mathrm{t}$
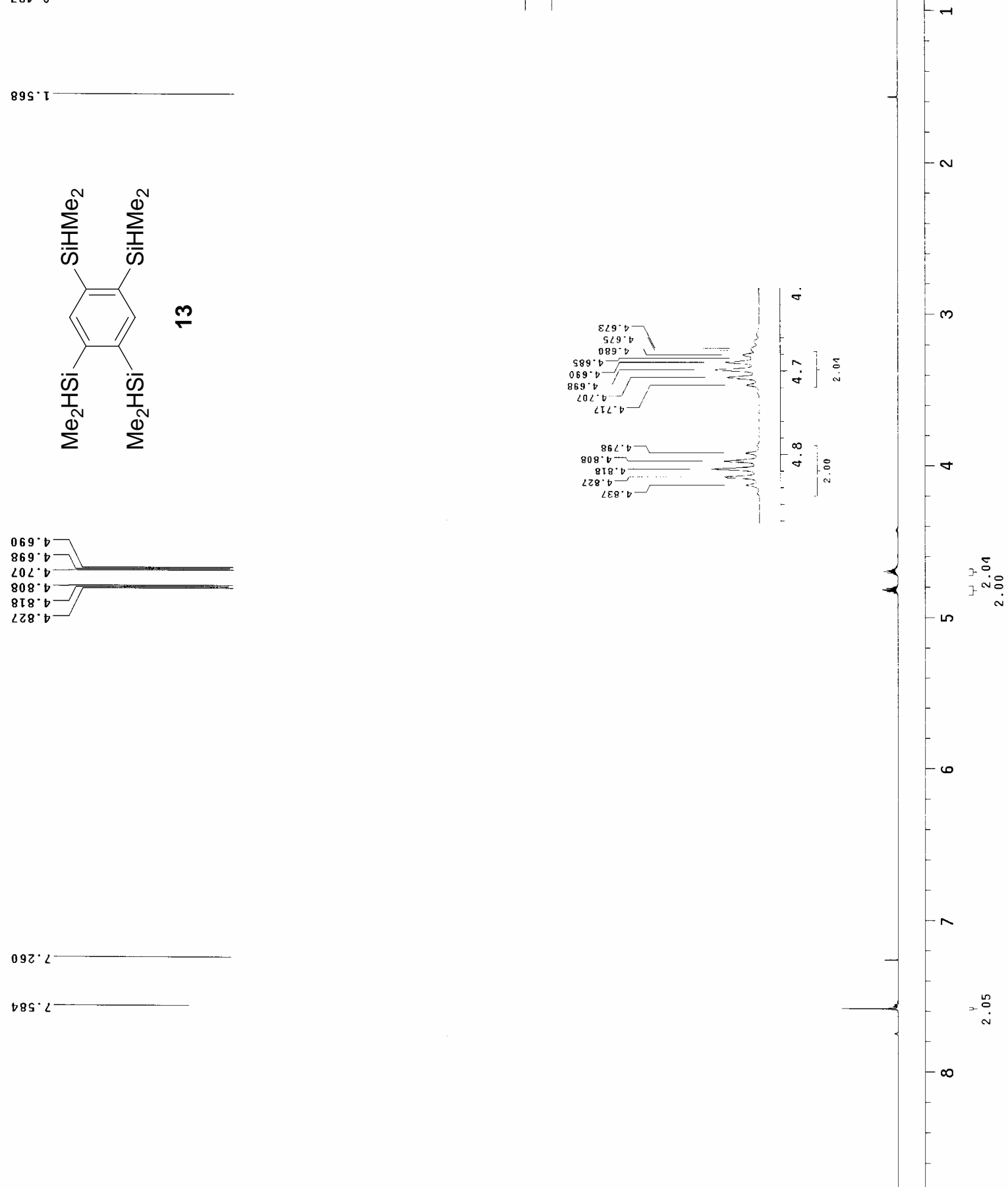

$092 \cdot$

$78 s^{\circ} 2$ 
Ablert W. M. Lee et al. - Supporting Information

${ }^{13} \mathrm{C}$ spectrum of $\mathbf{1 3}$

SI $6 \cdot$ S $I-$

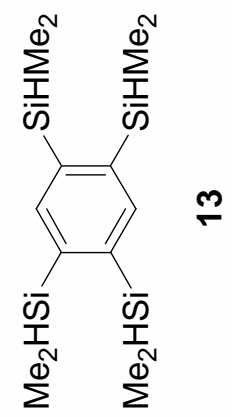

$089^{\circ} 92$

$\varepsilon \tau \varepsilon \cdot \angle L$

$s 乙 z \cdot t \varepsilon t$

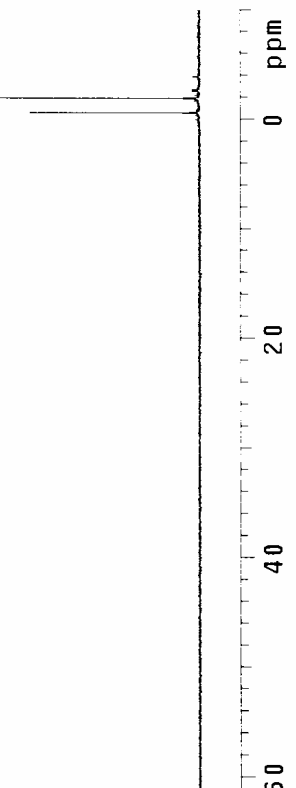

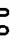

:

$\underset{\sim}{-}$

$\angle S Z \cdot 9 b \tau$

$\varepsilon Z I \cdot Z S I$

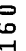


Ablert W. M. Lee et al. - Supporting Information

${ }^{1} \mathrm{H}$ NMR spectrum of $\mathbf{1 4}$

$800^{\circ} 0^{-\cdots}-$

$\triangleright 8 \varepsilon \cdot 0-$

$69 \mathrm{~S} \cdot \mathrm{I}$

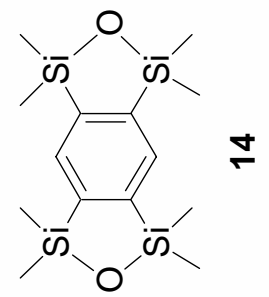

$092 \cdot 2$

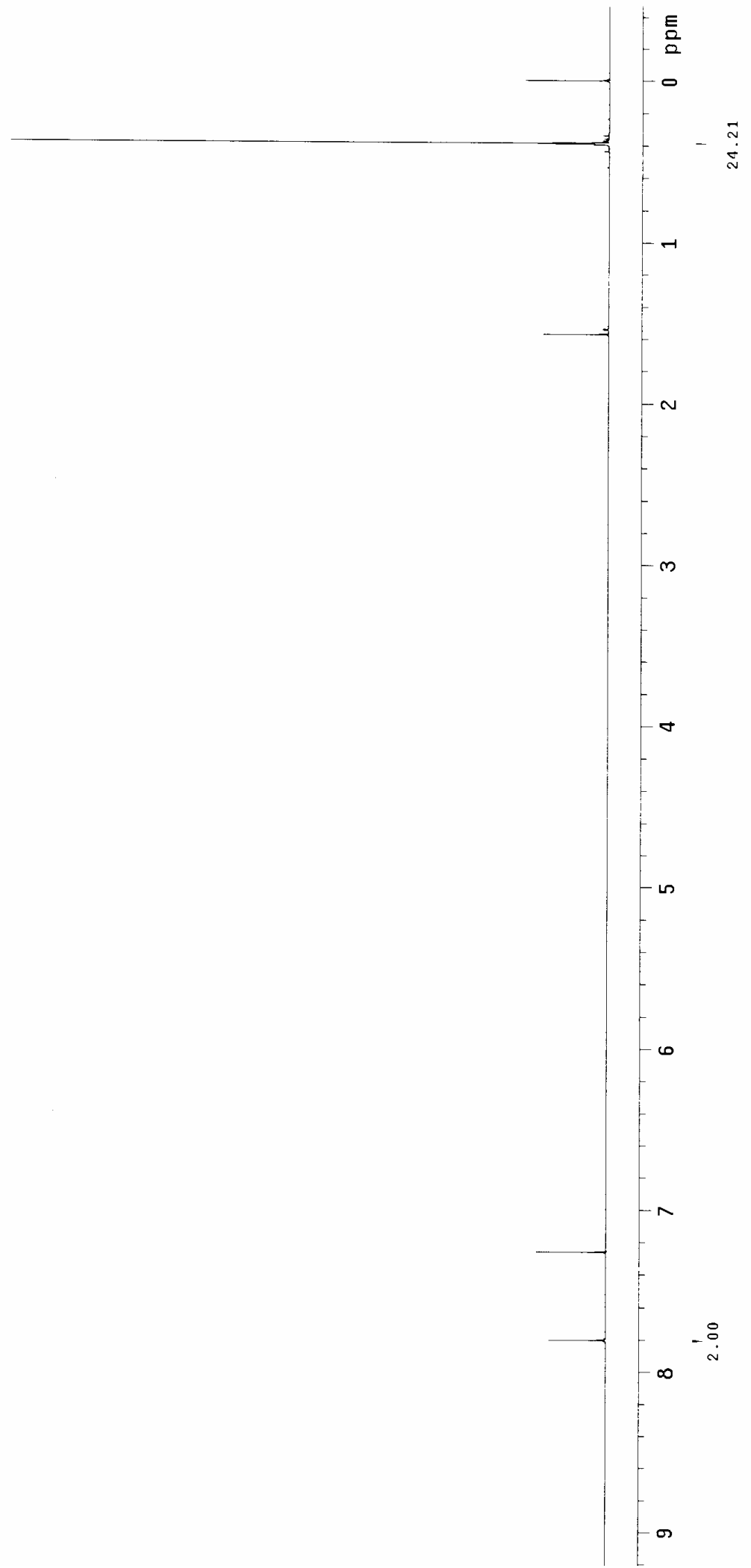


Ablert W. M. Lee et al. - Supporting Information

${ }^{13} \mathrm{C}$ NMR spectrum of 14

$090 \cdot \tau-$

$1=$

089.92

$\mathcal{0} \varepsilon \mathbf{\varepsilon} \varepsilon \cdot \ll<$

$\bar{n}_{0}^{\infty}$

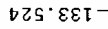

SDS' $8 D I$

\section{틈}

in

$e^{2}$

$\stackrel{0}{-0}$ 
Ablert W. M. Lee et al. - Supporting Information

${ }^{1} \mathrm{H}$ NMR spectrum of $\mathbf{1 5}$
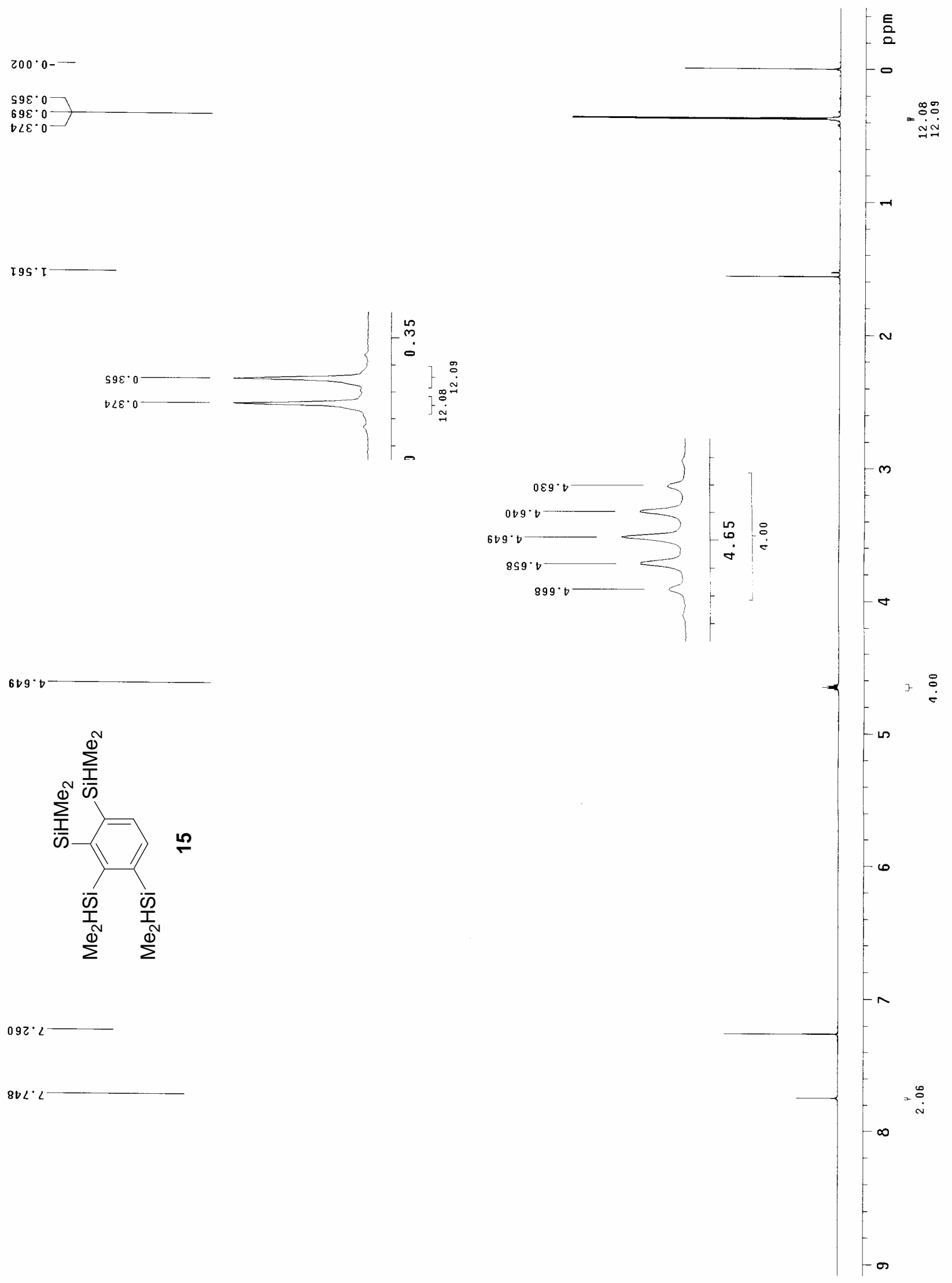

$092 \cdot \iota-$

$80 L \cdot L$

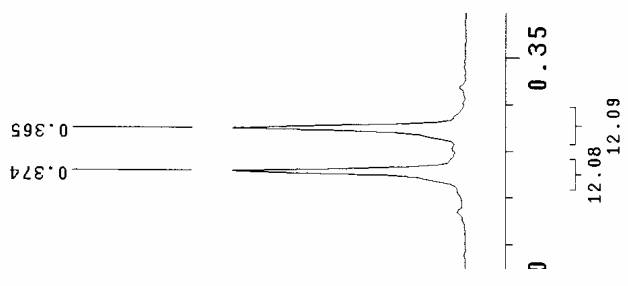

$6+9 \cdot 6$ 
${ }^{13} \mathrm{C}$ NMR spectrum of 15
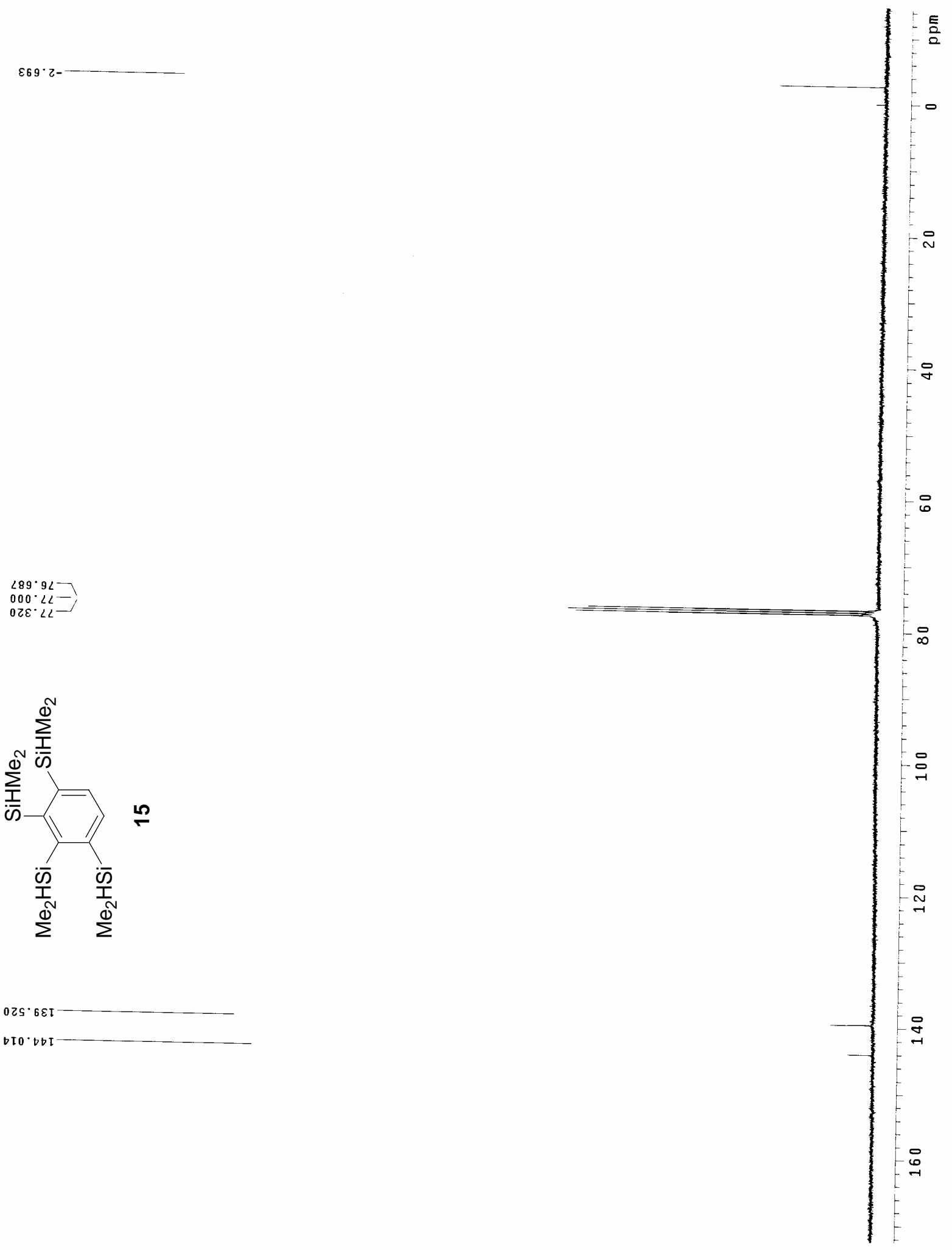
Ablert W. M. Lee et al. - Supporting Information

${ }^{1} \mathrm{H}$ NMR spectrum of $\mathbf{1 6}$

$08 \varepsilon^{\circ} \cdot 0$

$92 \mathrm{~b} \cdot 0$

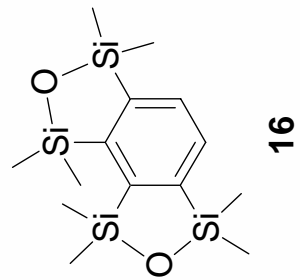

$\operatorname{sig}{ }^{\circ} \angle$

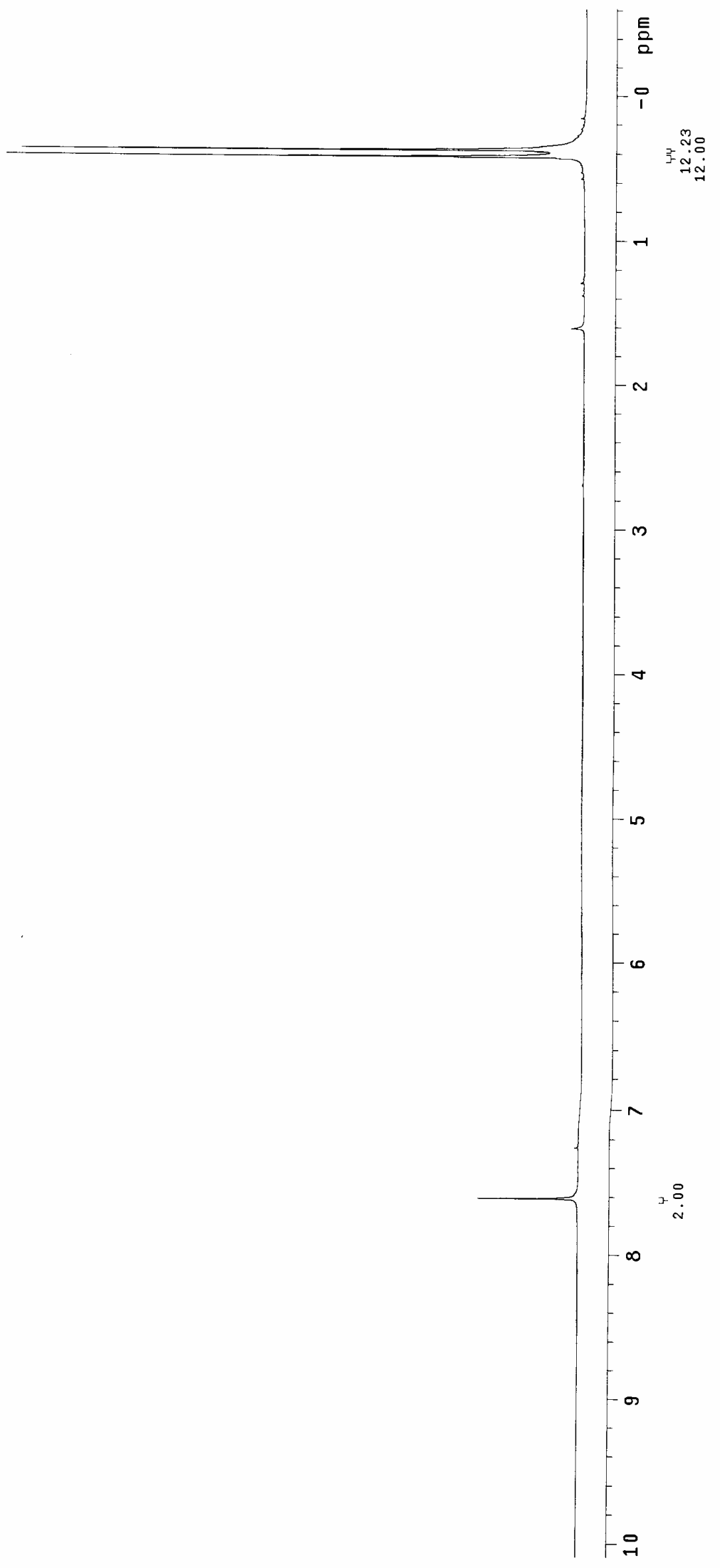


${ }^{13} \mathrm{C}$ NMR spectrum of $\mathbf{1 6}$
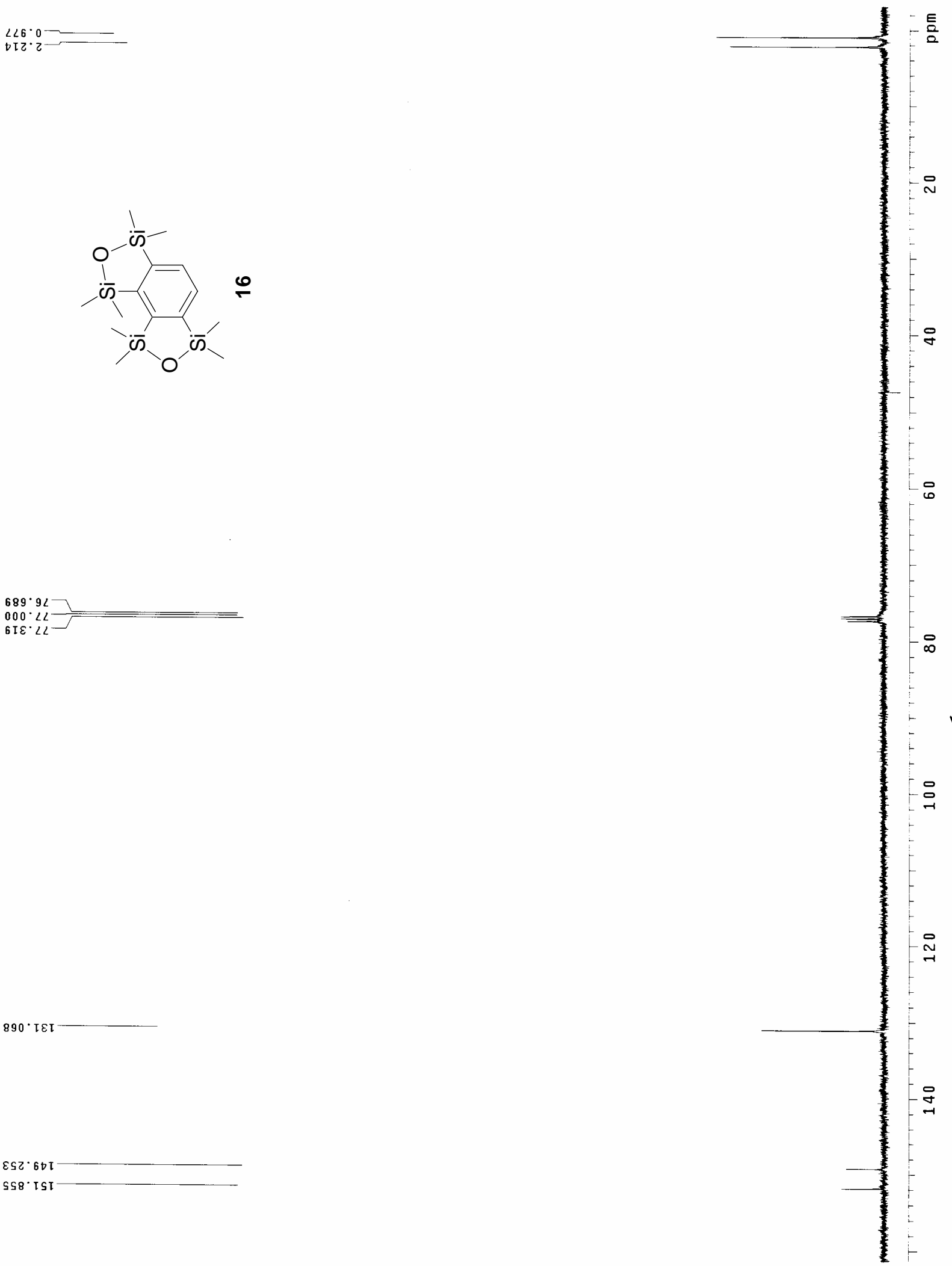

$\varepsilon s 2 \cdot 6 \mathrm{D}$

$689 \cdot 9 L$

6I $\varepsilon^{\circ} \angle L$

$\stackrel{9}{9}$

$890^{\circ} \mathrm{L} 8 \mathrm{I}$

รง8. โรเ 
Ablert W. M. Lee et al. - Supporting Information

${ }^{1} \mathrm{H}$ NMR spectrum of 19a

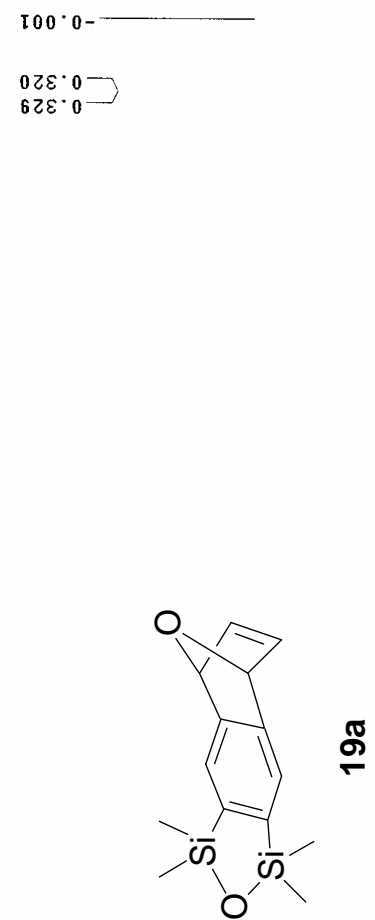

$+\frac{1}{a}$

$\frac{6}{2}$

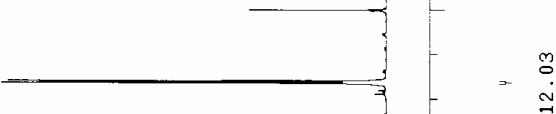

$\varepsilon 0 L^{\circ}$

$\stackrel{\nwarrow}{్}$

$\varepsilon \varepsilon_{0} \cdot$

$092 \cdot 2$

โZD०

$\cdot s$

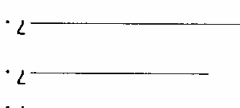

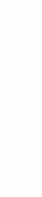

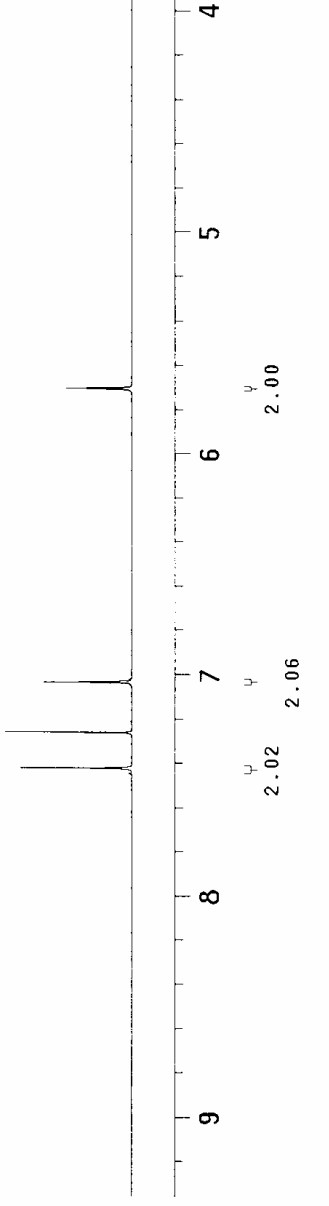


${ }^{13} \mathrm{C}$ NMR spectrum of 19a

$696 \cdot 0$
St0.

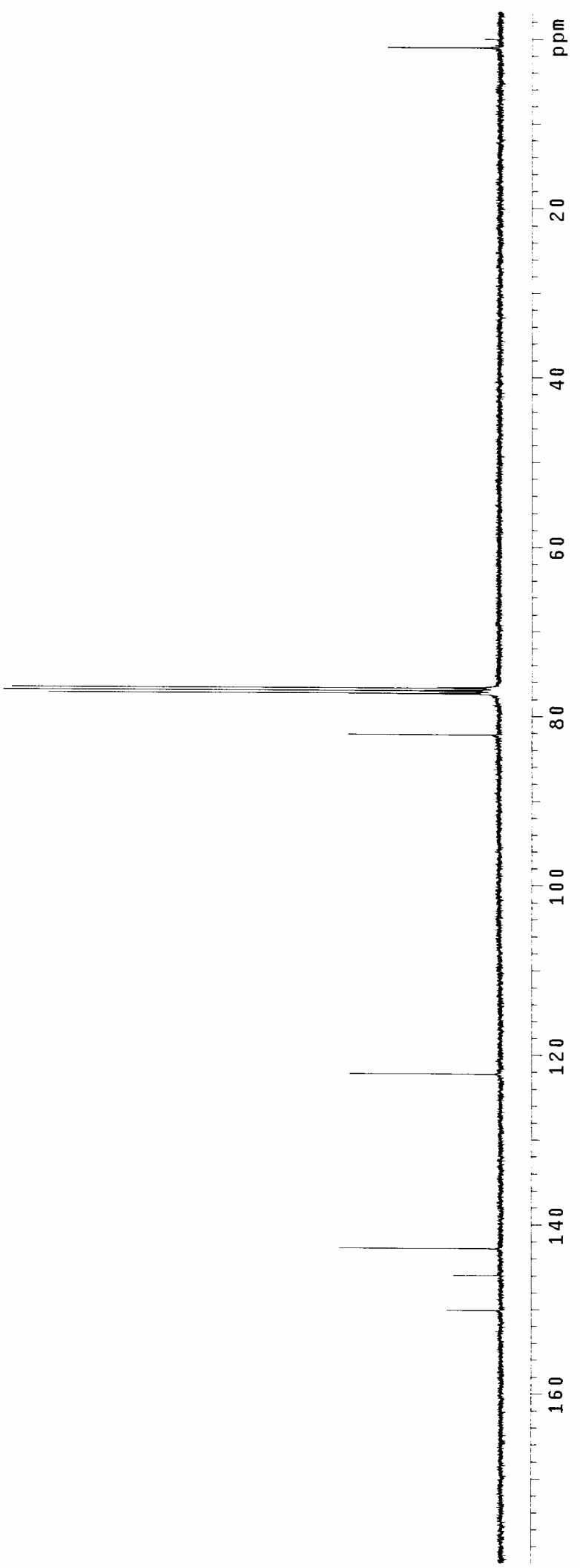

$089 \cdot 92$

$000 \cdot \angle L-$

$08 \mathrm{I} \cdot 28$

๑็

iक कर

$0 \tau Z^{\circ} 2 Z \tau$

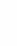

$6 \angle 0 \cdot 0 S I$

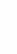




\section{${ }^{1} \mathrm{H}$ NMR spectrum of $\mathbf{1 9 b}$}
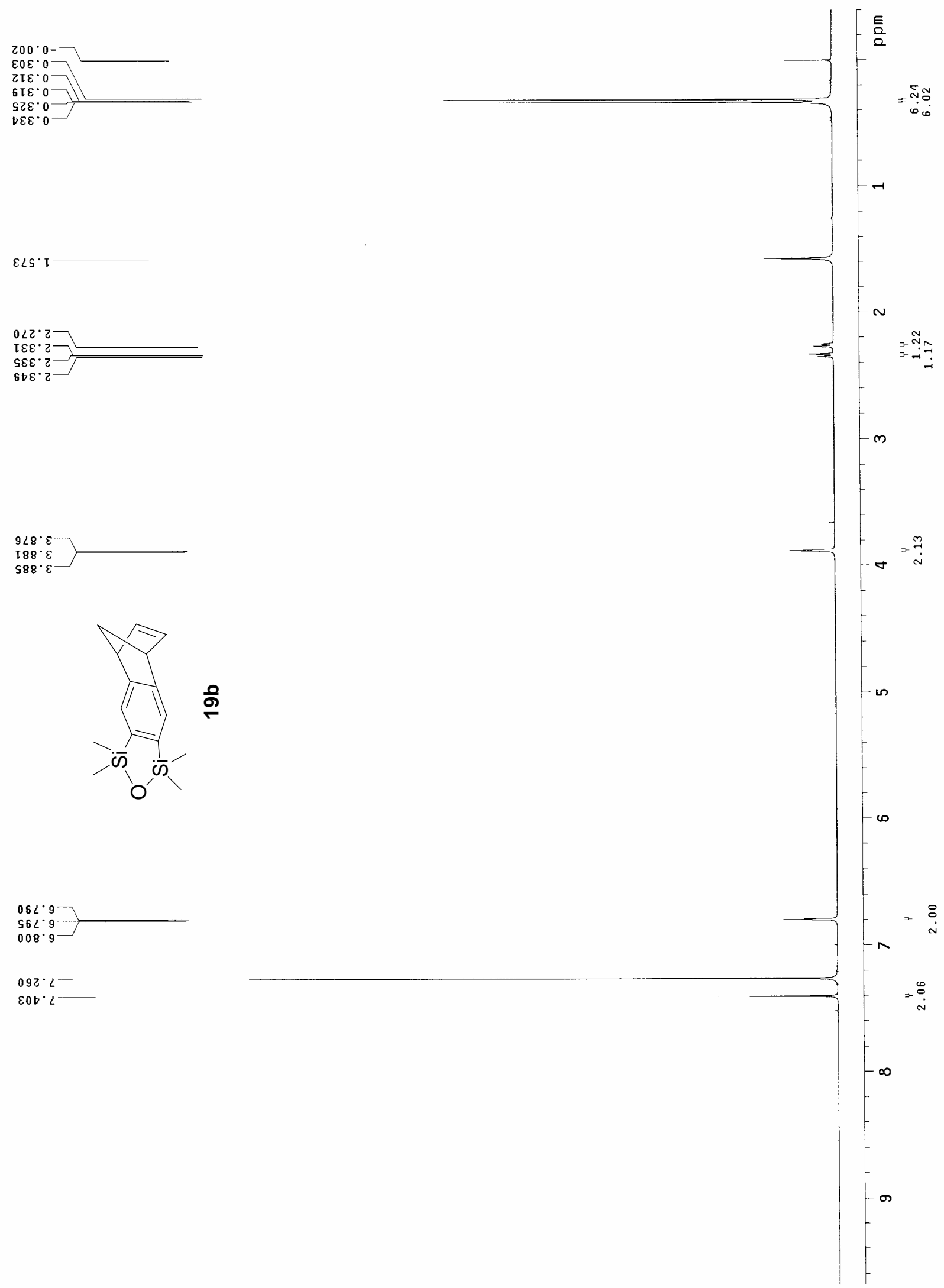

$9 \angle 8^{\circ} \varepsilon$

I $88^{\circ} \varepsilon$

I $\varepsilon \varepsilon \cdot 2$

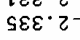

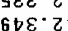

$\varepsilon \angle S \cdot I$

$06<\cdot 9-$

$56 L \cdot 9$

$092 \cdot 2-$

$\varepsilon 0 t \cdot 2$

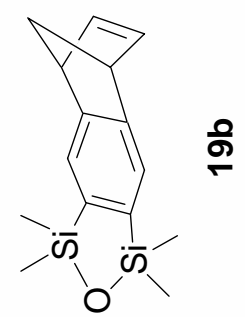


${ }^{13} \mathrm{C}$ NMR spectrum of $\mathbf{1 9 b}$

$\operatorname{t\in \mathcal {I}} \cdot \mathbf{I} \cdot$

$D \angle T \cdot 0 S$

799.69

$189 \cdot 9<-$

$000 \cdot 21$

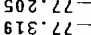

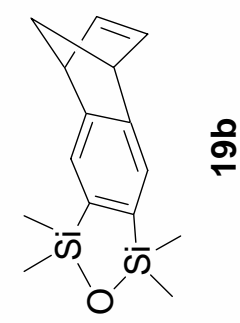

$889^{\circ} \varepsilon Z \tau$

윽

$216 \cdot 2 b t$
$6 S S^{\circ} b D t$

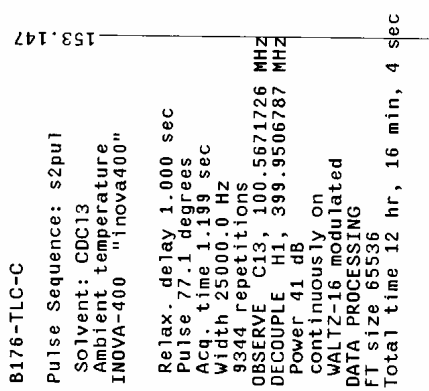

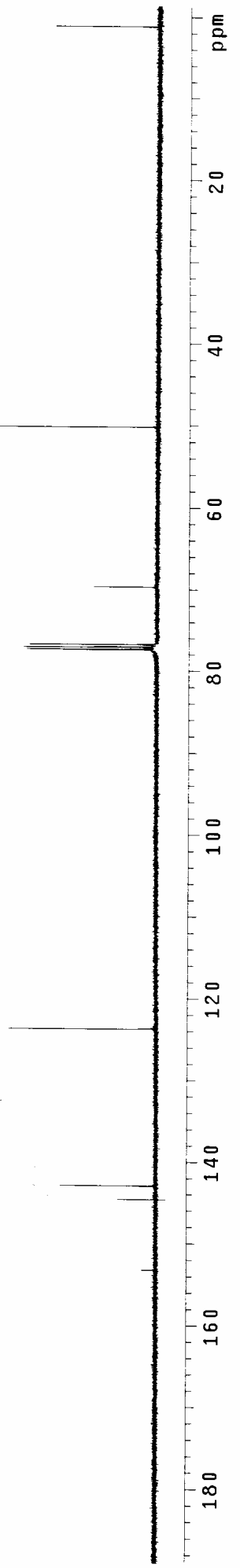


${ }^{1} \mathrm{H}$ NMR spectrum of 19c
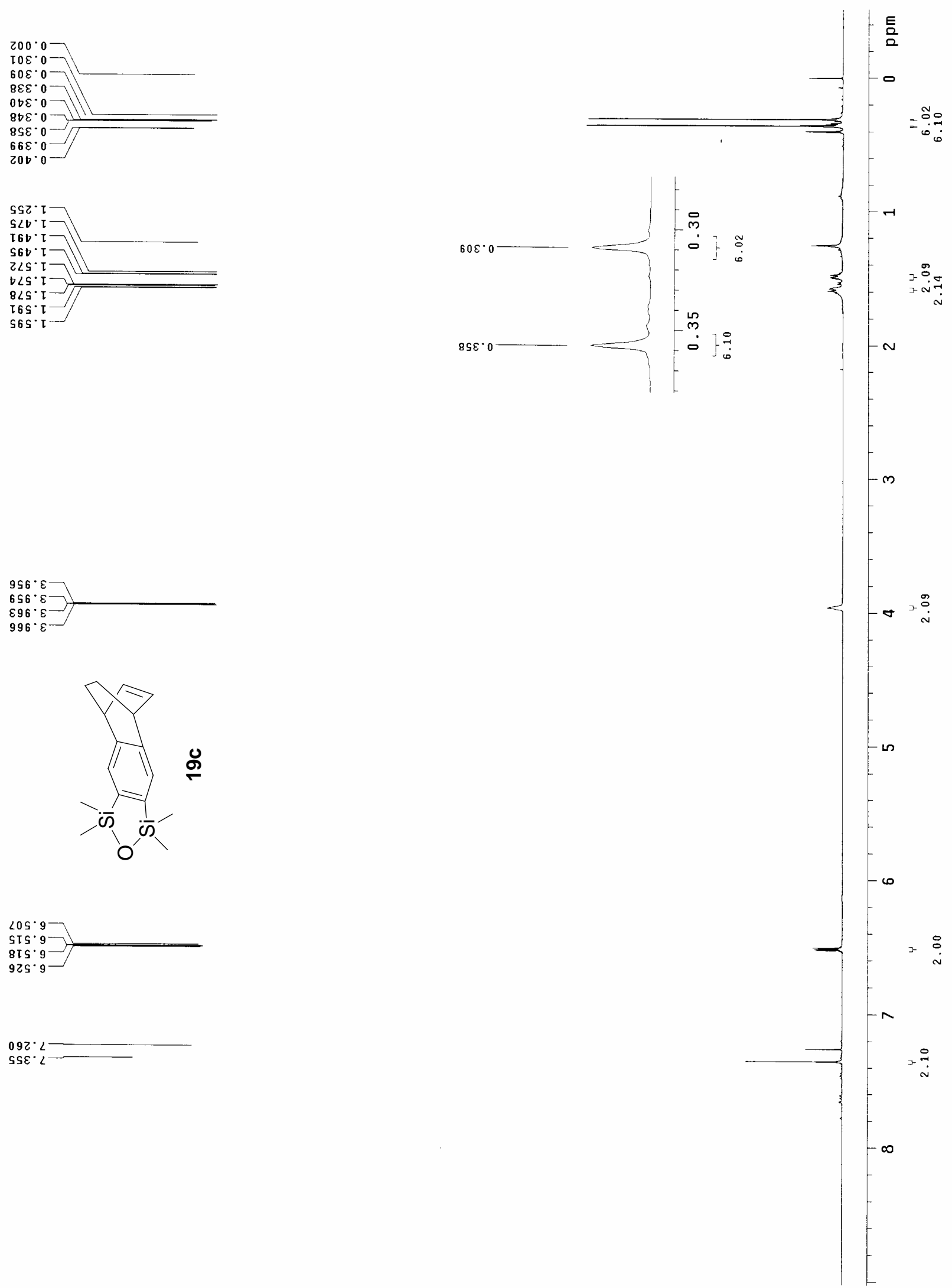

$092 \cdot L$
$\mathrm{SSE} \cdot L$

$9 S 6^{\circ} \varepsilon$

$6 \mathrm{S6} 6^{\circ} \varepsilon$

$\varepsilon 96^{\circ} \cdot \varepsilon$
$996^{\circ} \varepsilon$

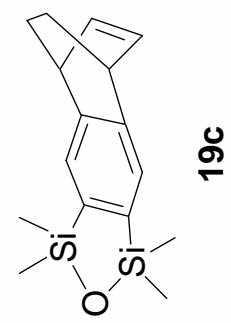

$\stackrel{9}{9}$

$\angle O S^{\circ}$
SIS

SIS.
$8 I S \cdot 9$

925. 9

ำ

$\frac{5}{2.2}$ 
${ }^{13} \mathrm{C}$ NMR spectrum of $19 \mathrm{c}$

${ }_{9 \varepsilon \mathcal{L}}^{\operatorname{Lst} \cdot \mathrm{I}-}$

I09. 2 Z

$D 90 \cdot 0 t$

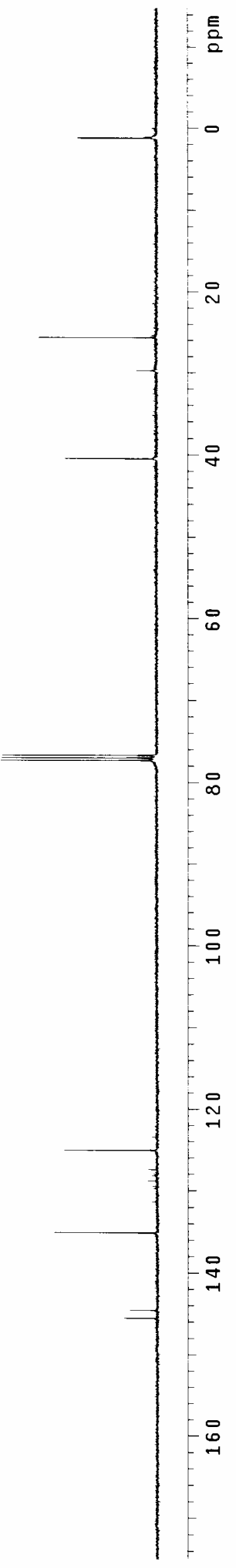

$\angle 89^{\circ} 9 \angle$

000.22

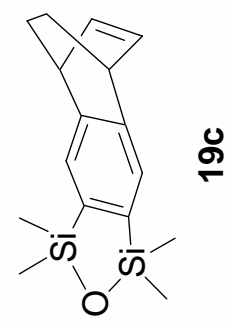

๑

$s 20 \cdot s z \mathbf{I}$

$S 60.5 E I$

$0 \angle S^{\circ} \cdot D t$

9TS. SDT 
Ablert W. M. Lee et al. - Supporting Information

${ }^{1} \mathrm{H}$ NMR spectrum of 19d
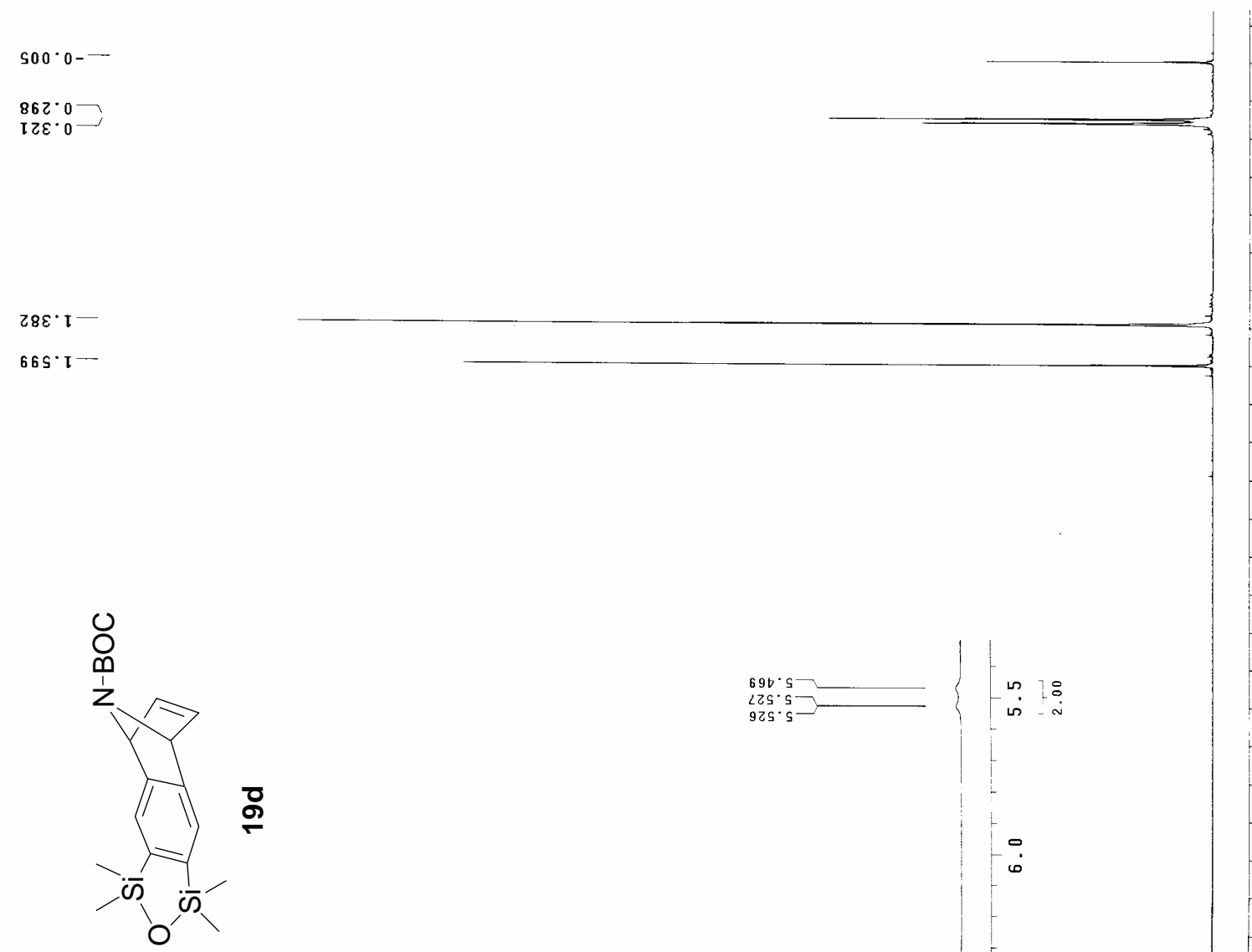

$\frac{\mathrm{E}}{2}$

$=\stackrel{\infty}{\substack{n\\}}$

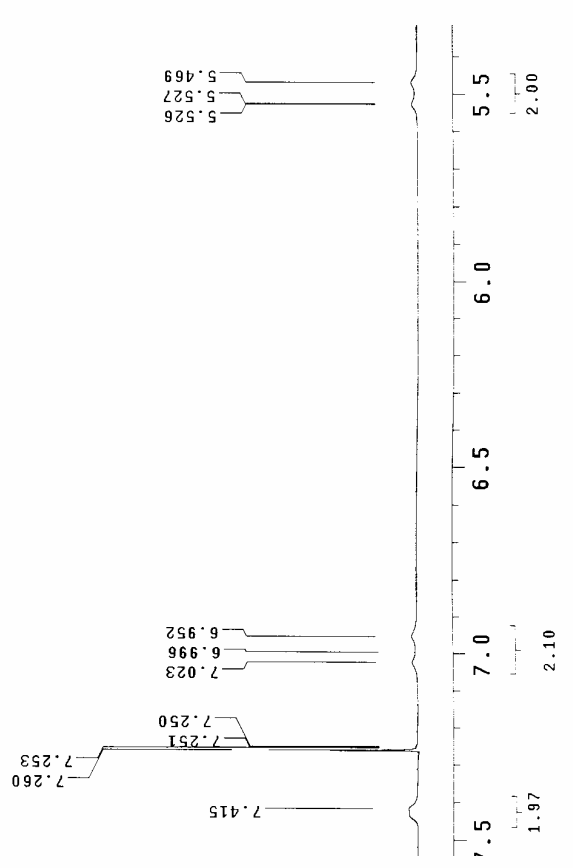

$m$

$092 \cdot\llcorner-$

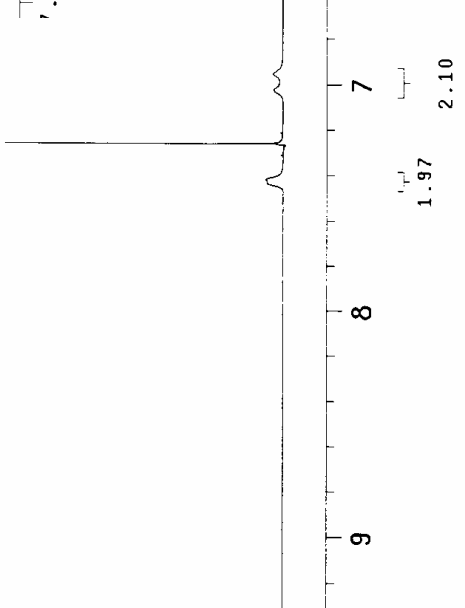


${ }^{13} \mathrm{C}$ NMR spectrum of $\mathbf{1 9 d}$
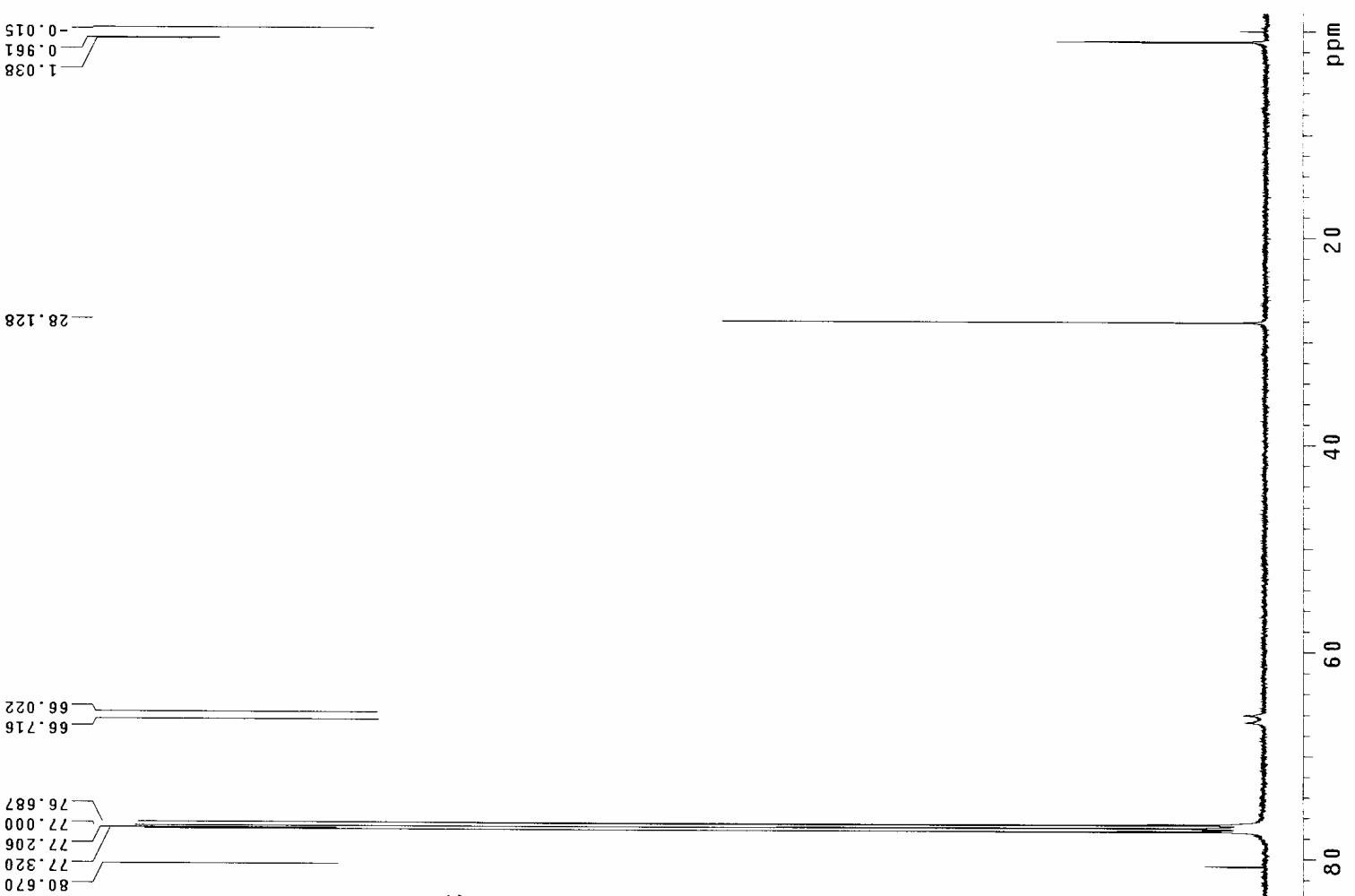

$220 \cdot 99$

$9 โ<\cdot 99$

$\angle 89^{\circ} 9 L$

$900^{\circ} \angle L$

$02 \varepsilon^{\circ} \angle L$

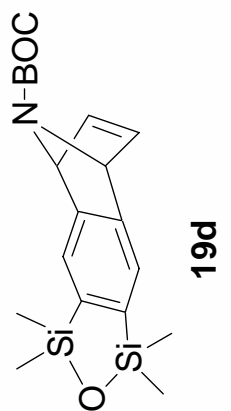

$900 \cdot z Z t$

$0 S 6 \cdot Z Z \mathbf{I}$

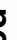

ort' $2 b i$

$80 S^{\circ} E D I$
$00 L$
$S D I$

$\varepsilon 9 \tau \cdot 6 b I$

$0 \varepsilon 0$. sSt 
${ }^{1} \mathrm{H}$ NMR spectrum of $\mathbf{1 9 e}$

$\varepsilon 10 \cdot 0-$
$\circ 00 \cdot 0-$

¿ा $\varepsilon \cdot 0$

$\triangle \varepsilon \varepsilon \cdot 0-$

$8 \varepsilon \varepsilon \cdot 0$

SSE. 0

$D D S^{\circ}$.

$80 S^{\circ} \cdot \mathrm{L}$

ISS. I

LLS. 1 -

$6 \angle S^{\circ} \mathrm{T}$
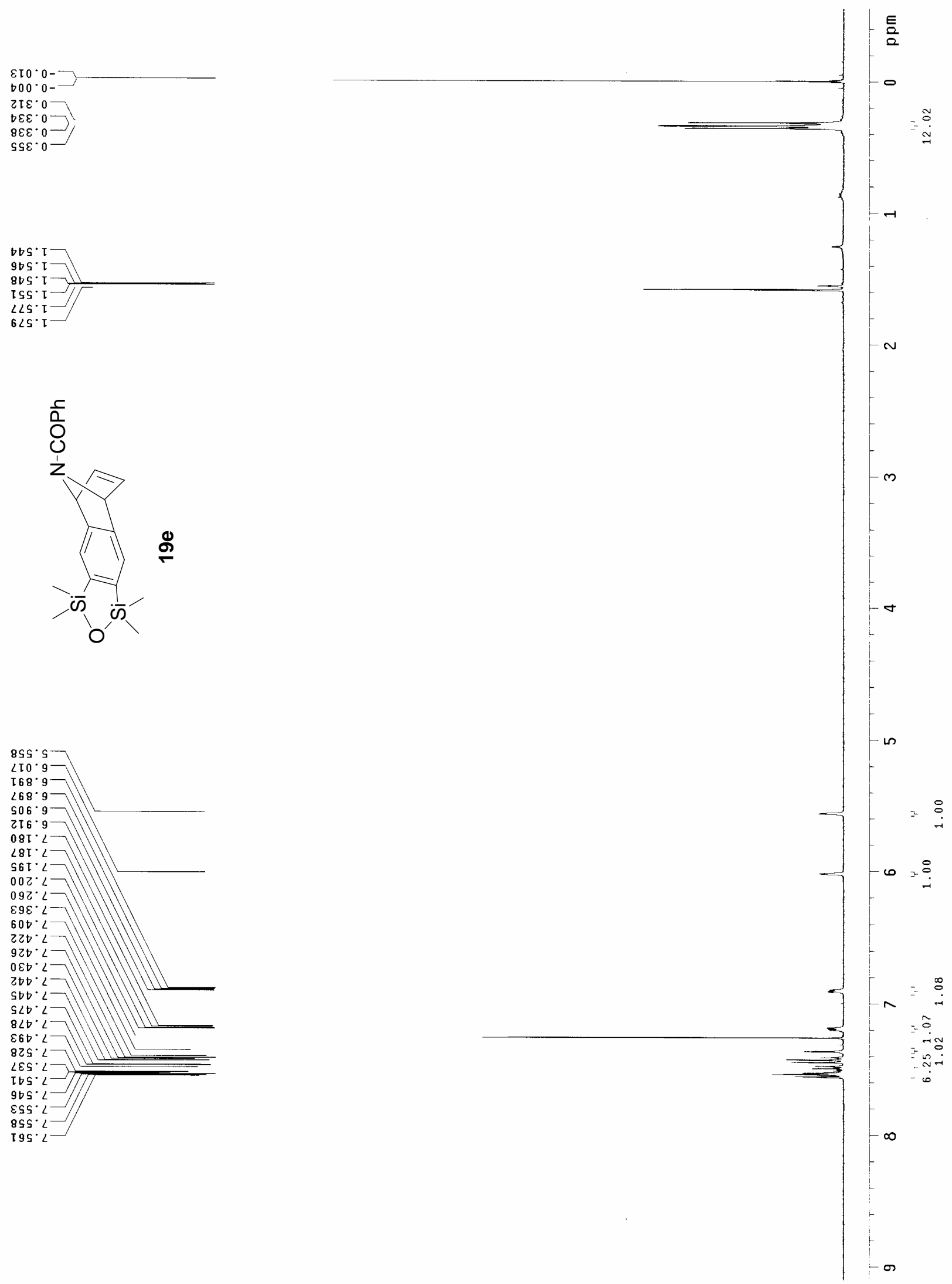
${ }^{13} \mathrm{C}$ NMR spectrum of $19 e$

SI $0 \cdot 0-$

$220 \cdot 1$

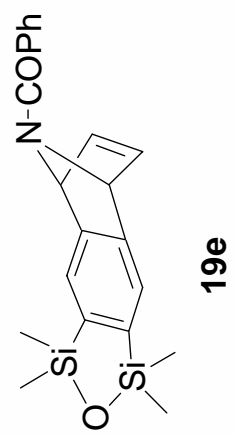

$\varepsilon \varepsilon \iota^{\circ} \varepsilon 9$

ZI I 89

$\angle 89^{\circ} 9 L$

$000 . \angle L$

$902 \cdot \angle 2$

$02 \varepsilon \cdot 2 L$

๑

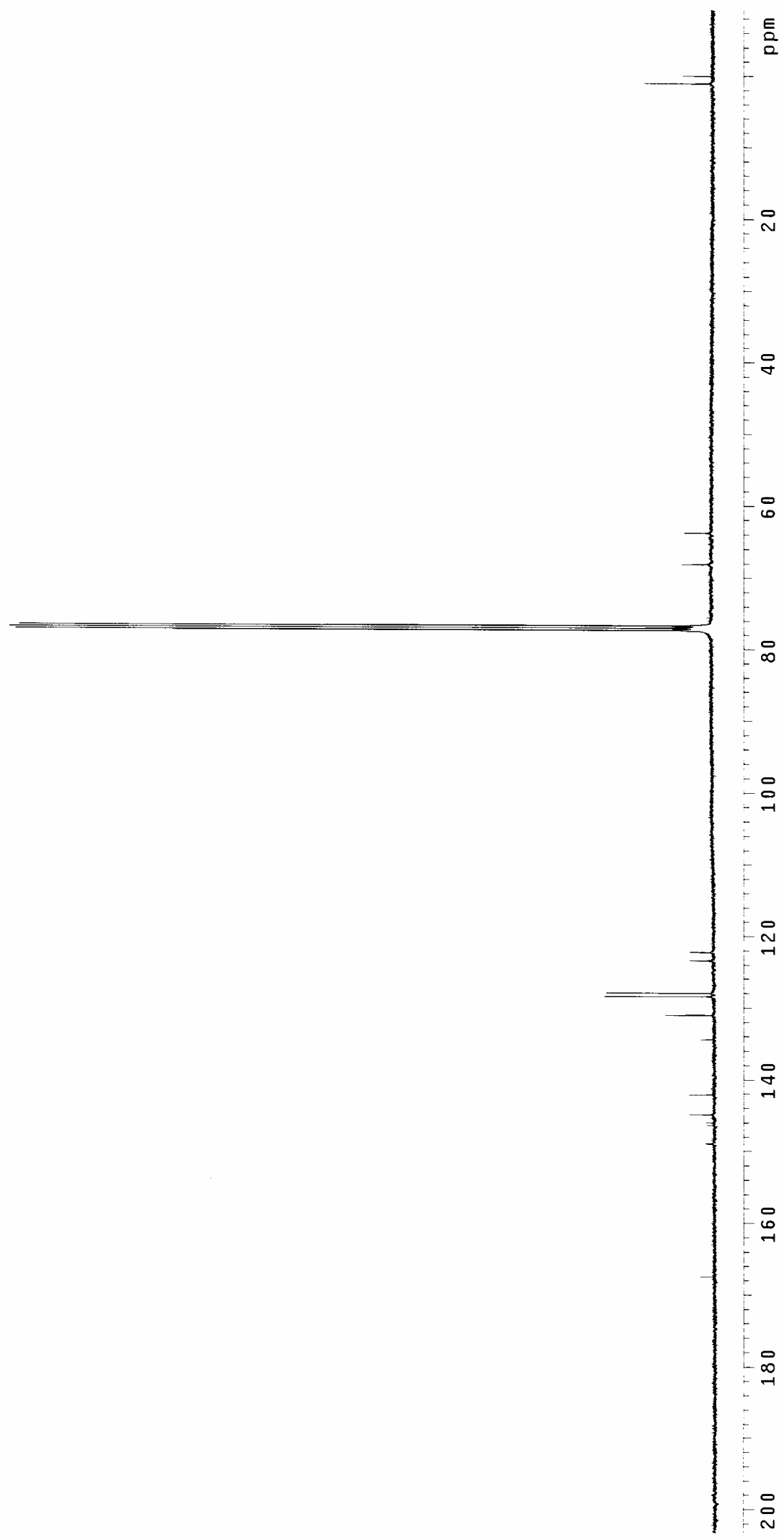

$0 \tau z \cdot \tau z \tau$

$80 \mathrm{~b} \cdot \varepsilon 2 \tau-$

$586^{\circ} \cdot 2 Z \tau-$

$0 \mathrm{St} \cdot 82 \mathrm{~T}$

$โ 20 \cdot \tau \varepsilon \tau$

$60 D^{\circ} \nabla \varepsilon I$

$890^{\circ} \mathrm{ZDT}$

$0 \varepsilon 8^{\circ} \mathrm{DOL}$

$b 56^{\circ} \mathrm{St} T$
$858^{\circ} 80 \mathrm{~T}$

$858^{\circ} 80 I$
$\angle S 6.80 T$

$S 9 D \cdot \angle 9 \tau-$

\section{(1)}


Ablert W. M. Lee et al. - Supporting Information

${ }^{1} \mathrm{H}$ NMR spectrum of 20a
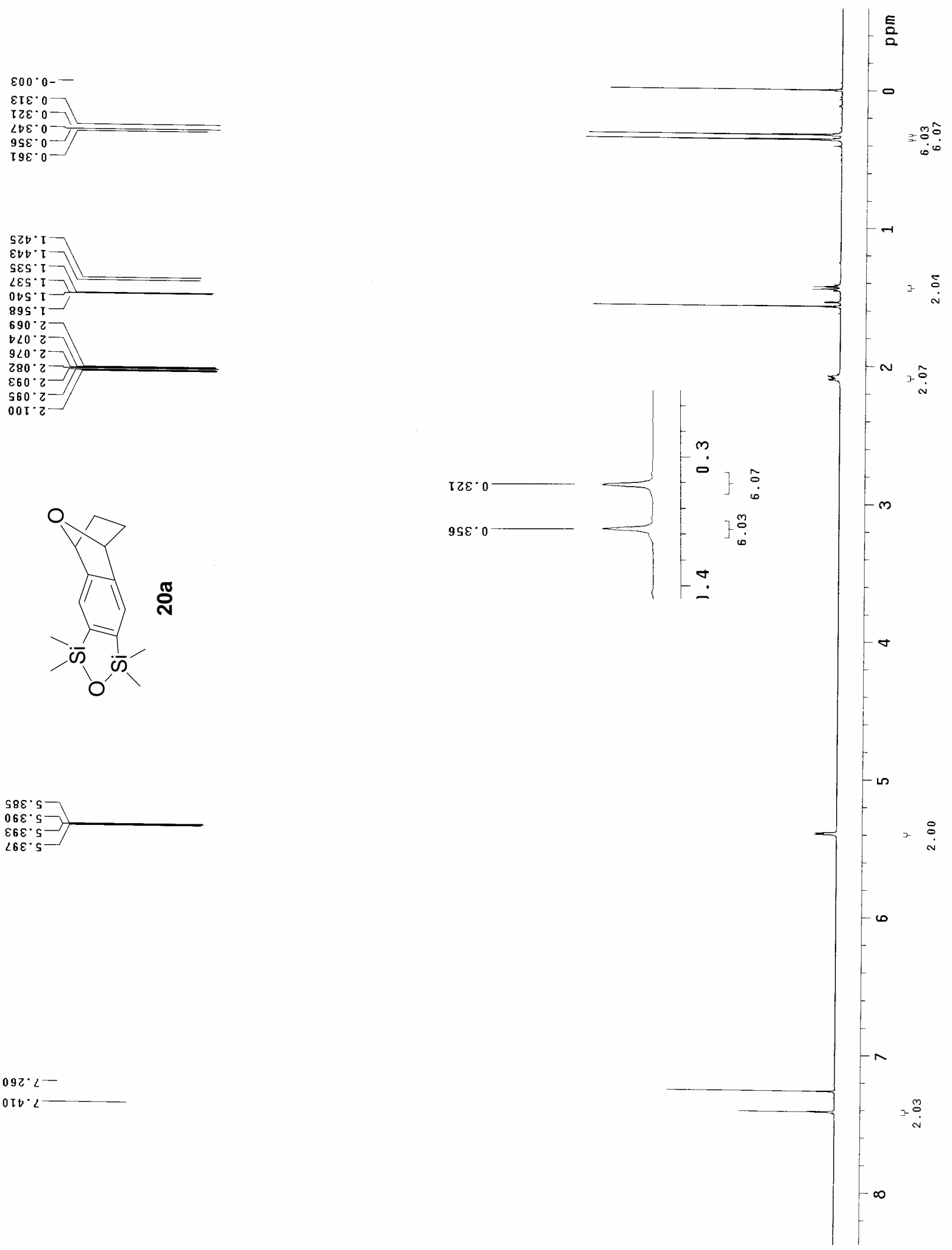

$092 \cdot 2^{-}$

0 It 2

S $8 \varepsilon^{\circ} \cdot s-$

$06 \varepsilon \cdot s$

$86 \varepsilon$

ฉั

$\stackrel{5}{\circ}$

\section{$\hat{0}$}

$60^{\circ} z-$
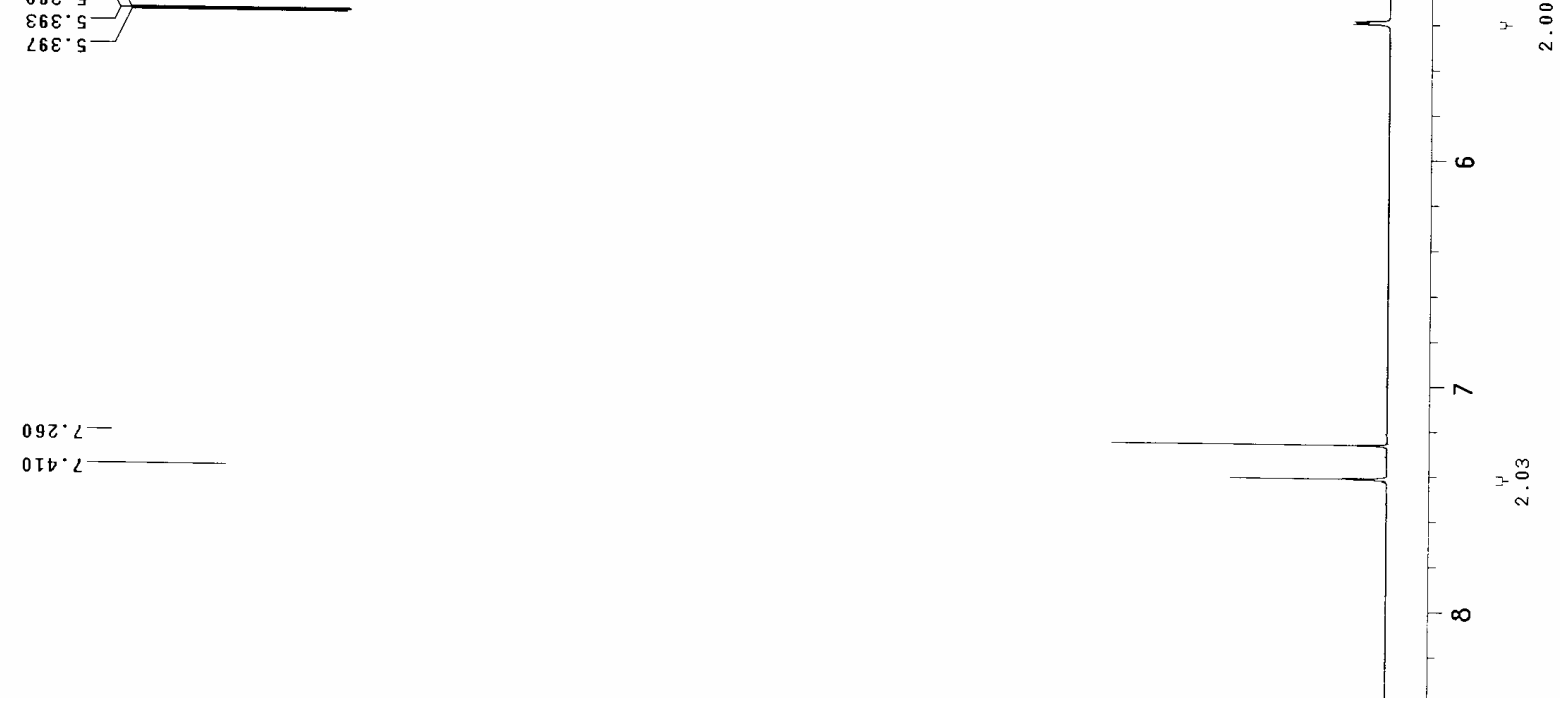
${ }^{13} \mathrm{C}$ NMR spectrum of $\mathbf{2 0 a}$

$886^{\circ} 0$

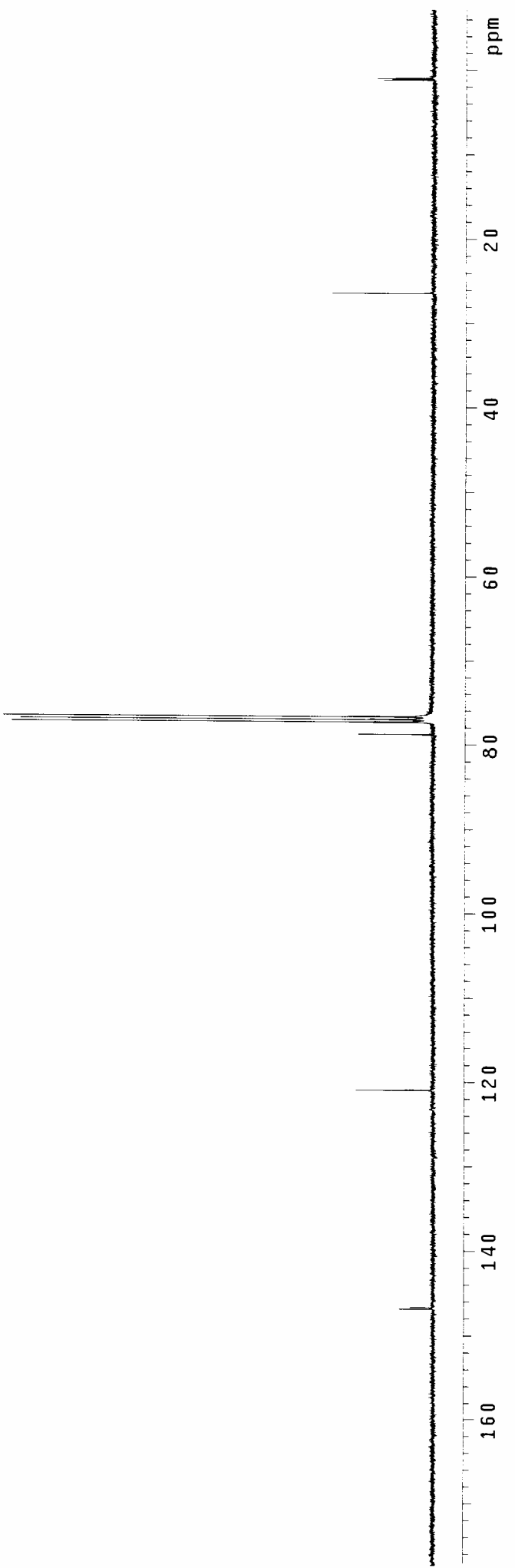

$\angle 89 \cdot 9<$

$02 \varepsilon \cdot 22$

$8 L L \cdot 8 L$

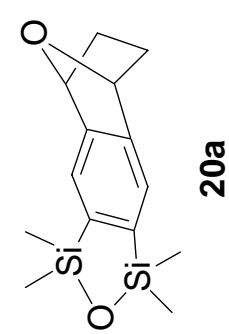

ฉั

$\varepsilon \tau 6 \cdot 02 \tau-$

$899 \cdot 9 \mathrm{DT}$

$\angle 98.9 b T$

赵

$\stackrel{\circ}{\square}$ 
${ }^{1} \mathrm{H}$ NMR spectrum of $\mathbf{2 0 b}$

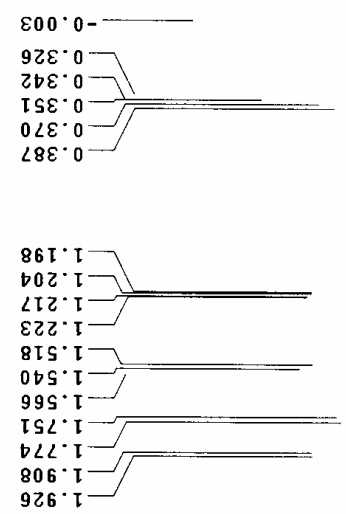

$2 ৮ \varepsilon \cdot \varepsilon$

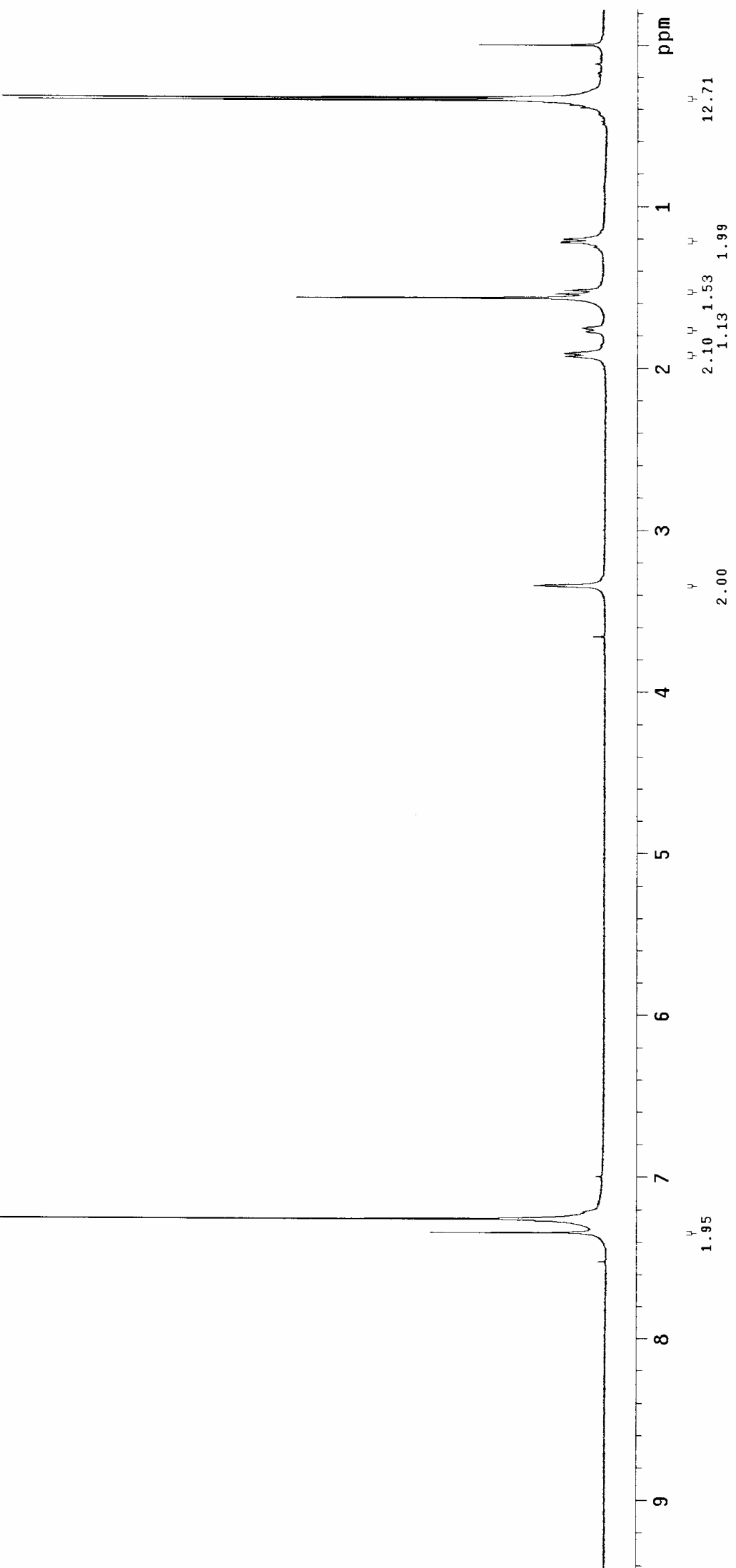


${ }^{13} \mathrm{C}$ NMR spectrum of 20b

$\operatorname{cor} \cdot \mathrm{i}$

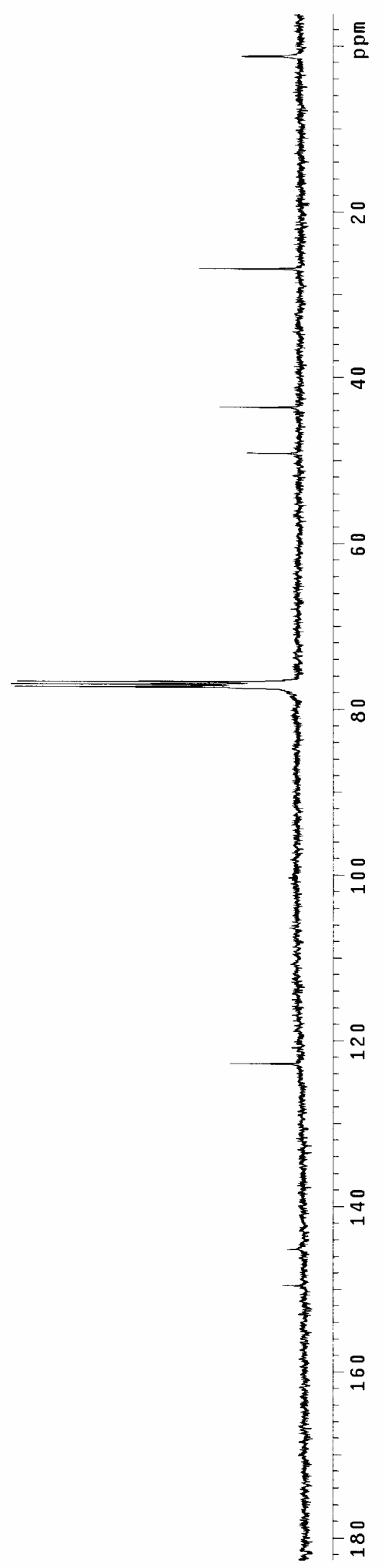

โ $89^{\circ} .9<$

$000 \cdot \ll-$

6 IE $L L$

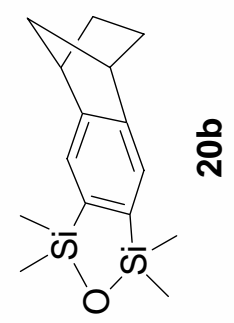

SSL ZZI

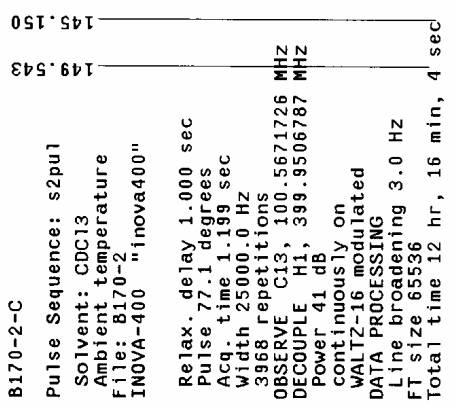


${ }^{1} \mathrm{H}$ NMR spectrum of 20c
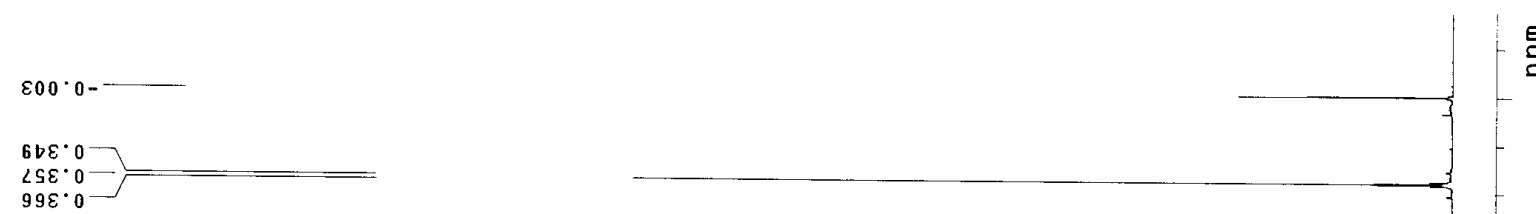

$6 b \zeta \cdot \tau$

200.1

$8 \angle S^{\circ} \mathrm{l}$

$S 8 L \cdot T$

$508 \cdot 1-$

$9 \angle I \cdot 2$

$\angle S E^{\circ} 0$

틈

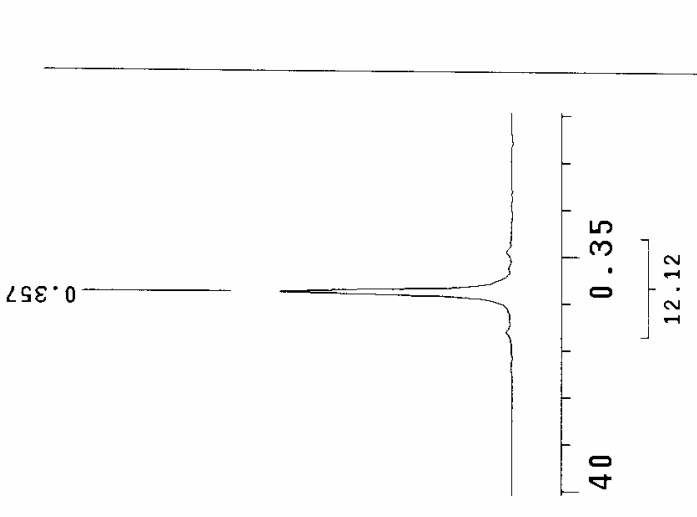

$\cong$

$986^{\circ} z^{-}$

:

$092 \cdot L$
$0 \varepsilon \varepsilon \cdot\llcorner$

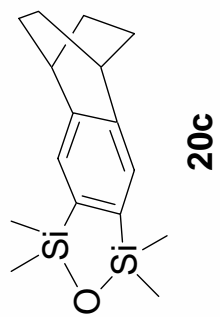

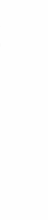


${ }^{13} \mathrm{C}$ NMR spectrum of 20c

$9 \varepsilon 2 \cdot \tau$

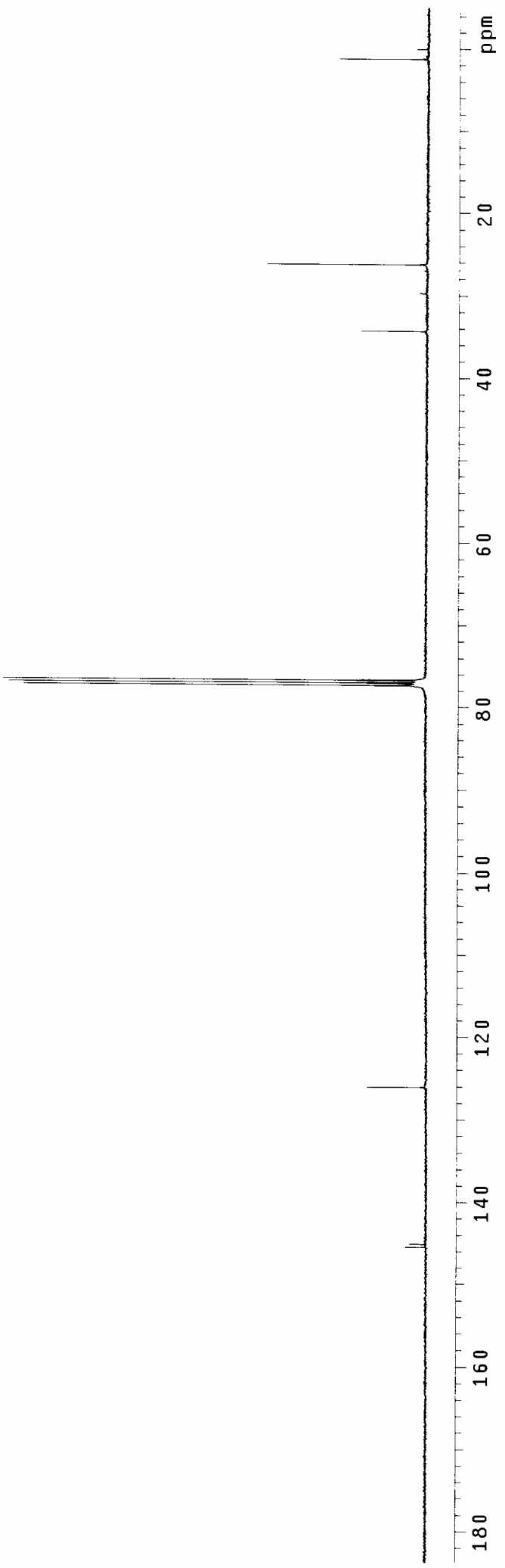

$089 \cdot 92$

$000^{\circ} 22$

$\varepsilon \tau \varepsilon \cdot \ll<-$

เฉZ・9z- -

$s 8 z \cdot b \varepsilon-$

ลั.

$s s 0 \cdot 92$

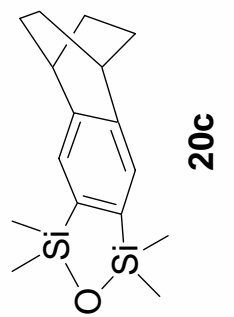

$\angle 60^{\circ} S 6 I$
$800^{\circ} .50 I$

$\stackrel{-g}{g}$

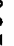

$\underset{\substack{\infty \\ \rightarrow}}{\rightarrow}$ 
${ }^{1} \mathrm{H}$ NMR spectrum of $\mathbf{2 0 d}$
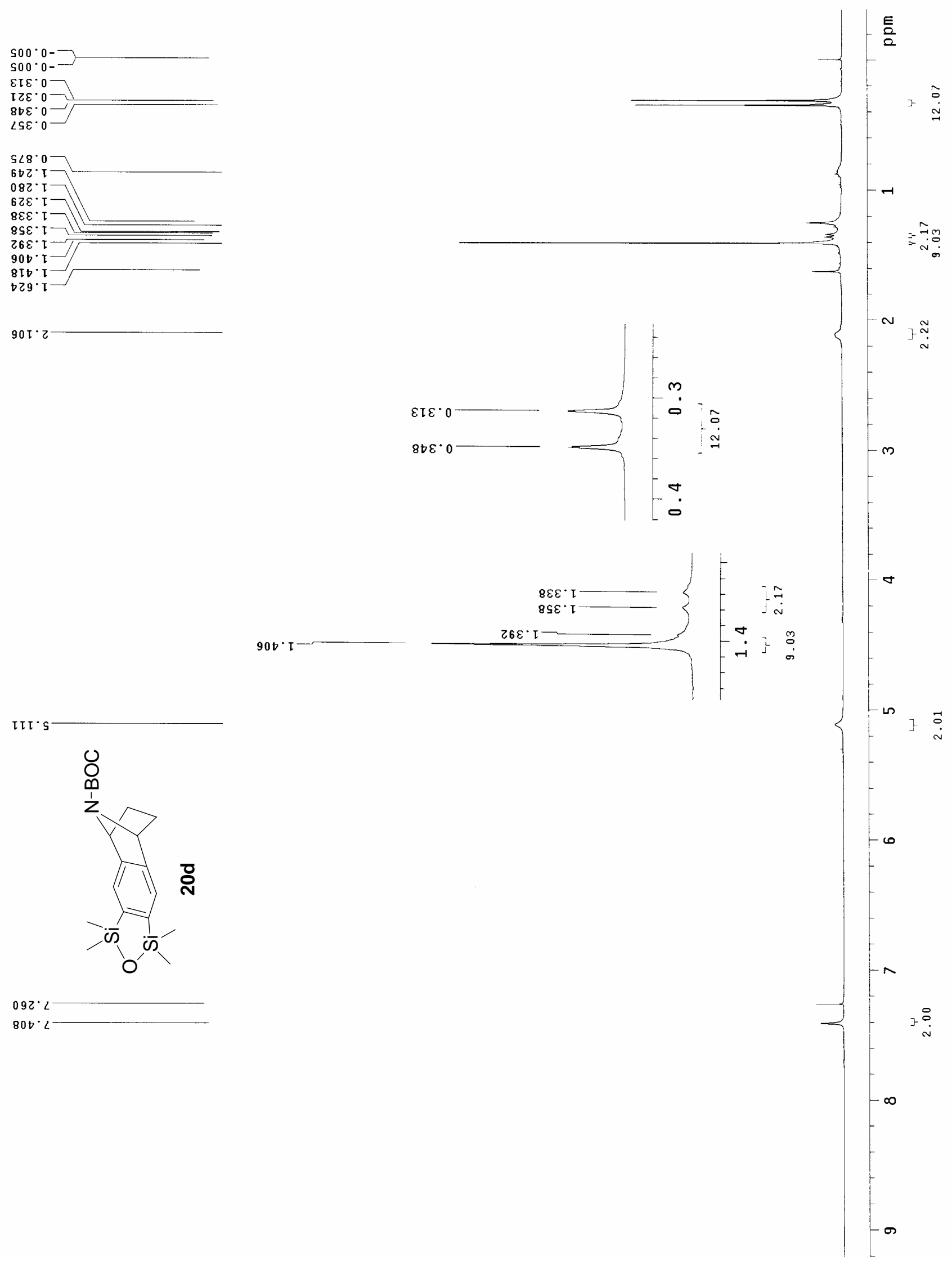

$092 \cdot L$

$80 b^{\circ} L$

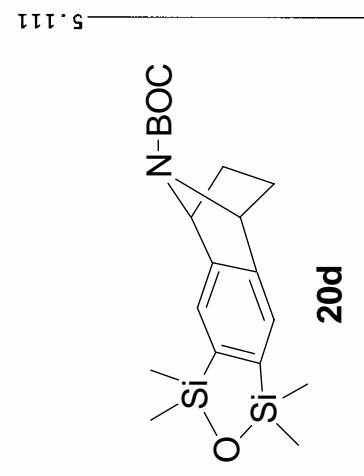

$90 b^{\circ} \tau$ 
${ }^{13} \mathrm{C}$ NMR spectrum of 20d

$\angle 66^{\circ} 0^{-}$
$591 \cdot 2$

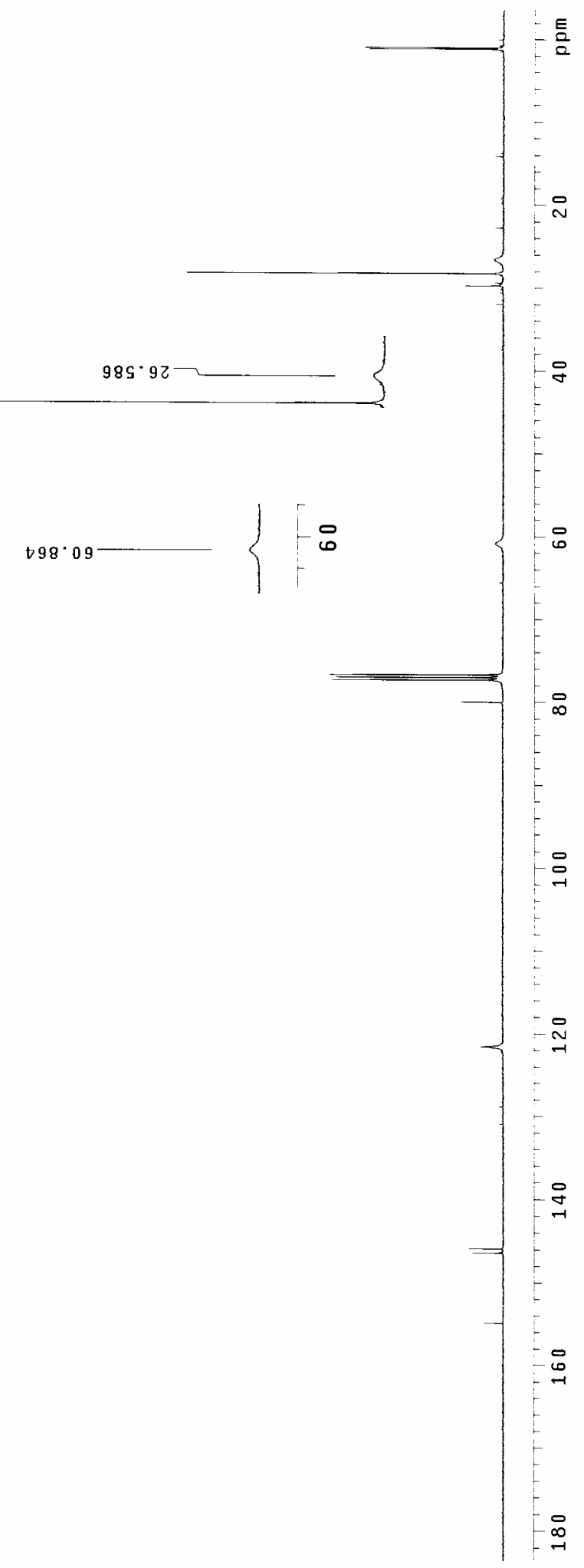

$\angle 89^{\circ} 9 L^{\circ}$

$900 \cdot 2 L$

$0 Z \varepsilon \cdot L L$

$S \angle G \cdot 62$

$812 \cdot 82-$

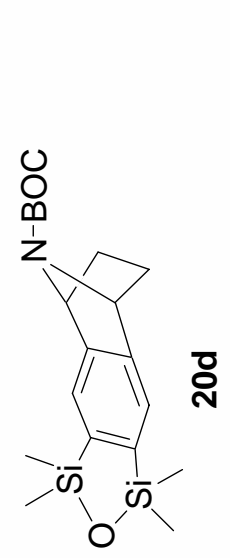

$268^{\circ} \cdot S D T$
$88 \varepsilon^{\circ} \cdot 90 I$

$\varepsilon 06 \cdot \nabla S I$ 
Ablert W. M. Lee et al. - Supporting Information

${ }^{1} \mathrm{H}$ NMR spectrum of 20e

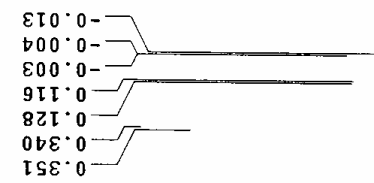

OLt $\tau$

$\varepsilon \varepsilon 0^{\circ} \mathrm{L}-$

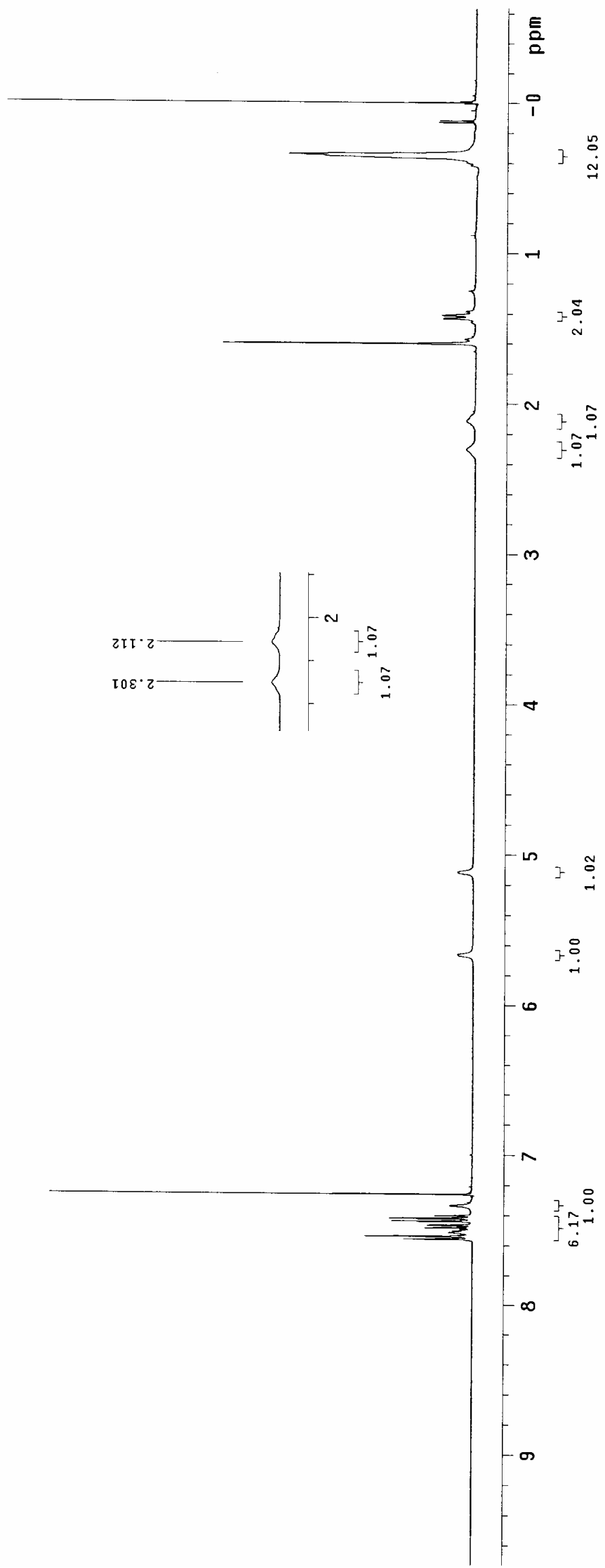

$099 \cdot \mathrm{s}$

$09 Z^{\circ} L$
$9 \varepsilon \varepsilon^{\circ} L$

$\angle 6 \varepsilon^{\circ} \angle$

SOD 2

DID $L$

$8 I t^{\circ} L$
$22 t^{\circ} L$

tED.

$\angle E D^{\circ} \angle$

GEt ${ }^{\circ} L$

$\rightarrow 90^{\circ}<-$

$\angle 90^{\circ}<$

$9 \angle b^{\circ} \angle$

$280^{\circ} \angle$

$280^{\circ} \angle$

${ }^{0 O S^{\circ}} \angle$

$\varepsilon \mathfrak{I S} \cdot\llcorner$

OES $L$

Ots.

$9 \triangleright S^{\circ}<$

ESS. 2

$\angle S^{\circ} L$

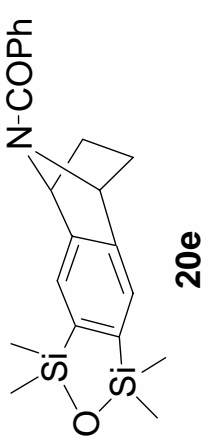

กั่

I $0 \varepsilon^{\circ}$ 
${ }^{13} \mathrm{C}$ NMR spectrum of $\mathbf{2 0 e}$

$\varepsilon 20^{\circ} 0_{-}-$

SI0.T

$59 S^{\circ} \mathrm{Sz}$

$\varepsilon S S^{\circ} 8 \mathrm{C}$

st $2 \cdot \varepsilon 9$

$089.9<$

266.92

$86 \tau \cdot L L$

$60 \tau \cdot \tau Z I$

$6 \mathrm{T0} \cdot 2 \mathrm{ZT}$

$\mathrm{DSG} \cdot \angle Z I$

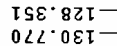

$880^{\circ} \varsigma \varepsilon I^{-}$

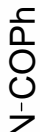

IZI'89I-

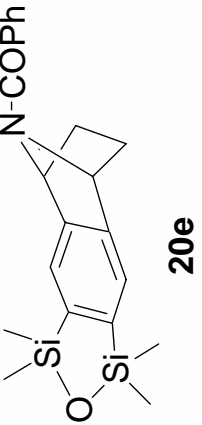

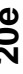


Ablert W. M. Lee et al. - Supporting Information

${ }^{1} \mathrm{H}$ NMR spectrum of 21
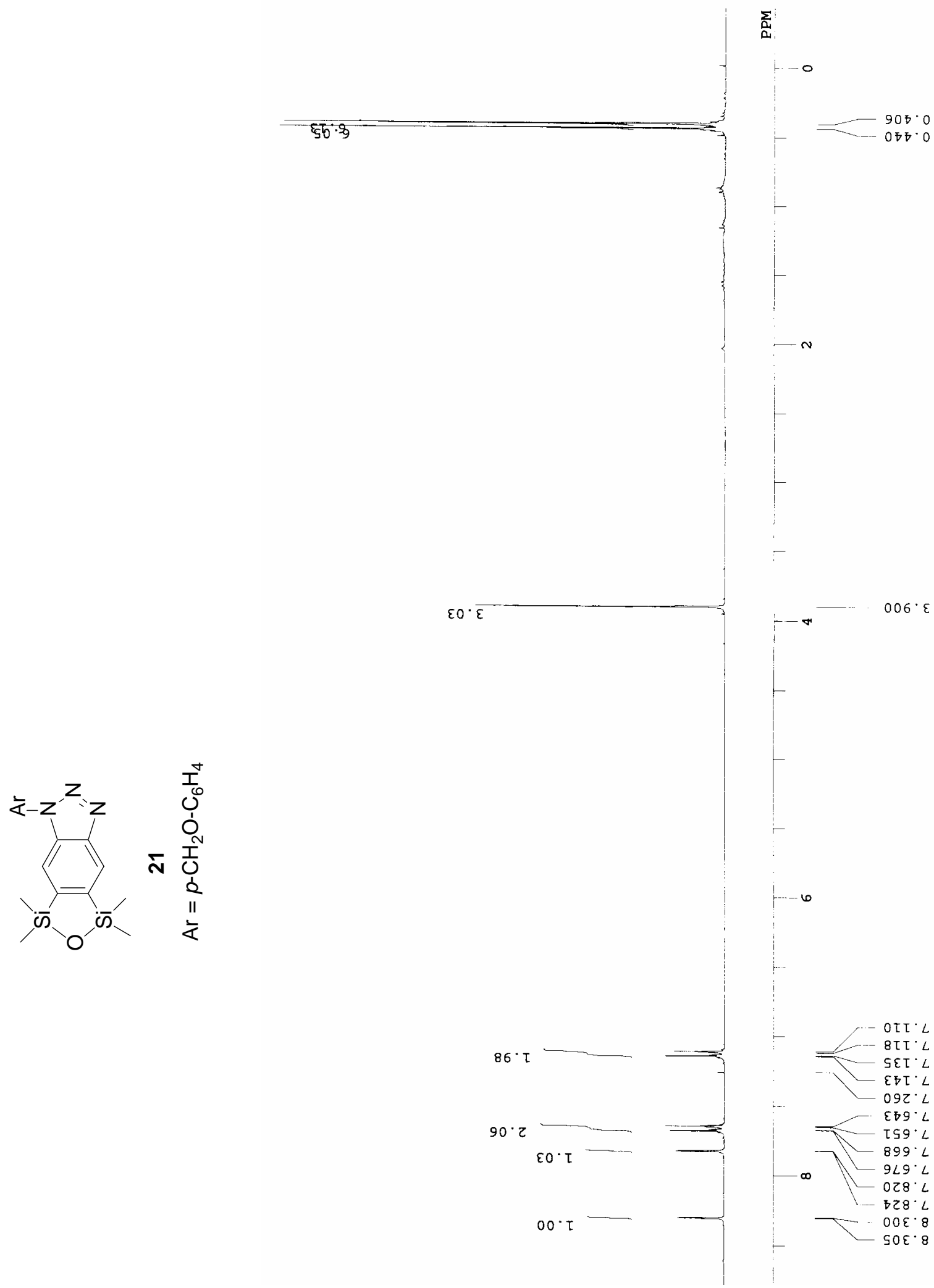
Ablert W. M. Lee et al. - Supporting Information

${ }^{13} \mathrm{C}$ NMR spectrum of $\mathbf{2 1}$
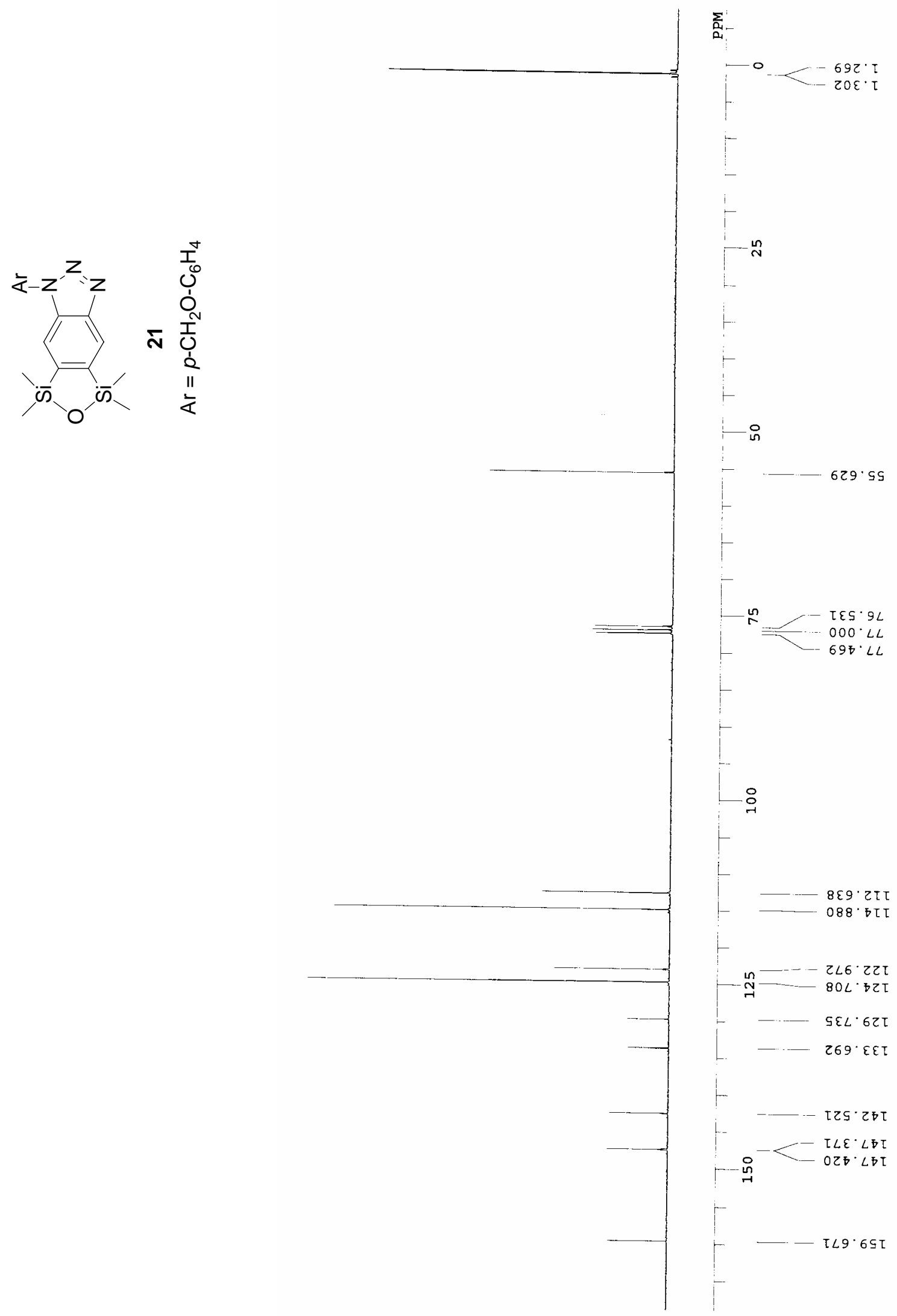
Ablert W. M. Lee et al. - Supporting Information

${ }^{1} \mathrm{H}$ NMR spectrum of 22
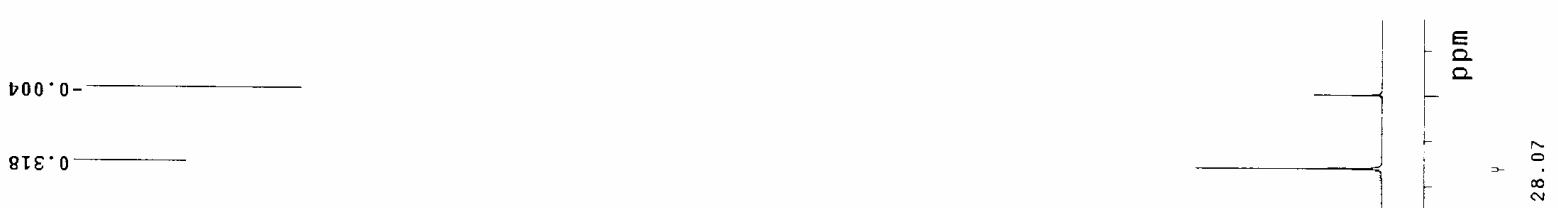

$\angle \angle S^{\circ} I$

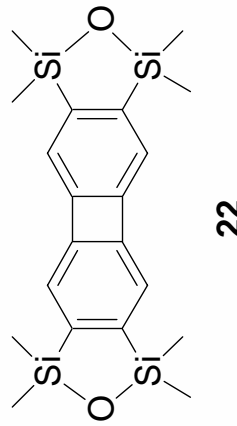

$0 \varepsilon 8 \cdot$

$09 Z^{\circ}<$

N

$\infty$

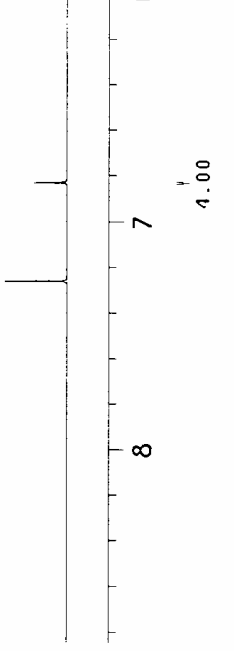


${ }^{13} \mathrm{C}$ NMR spectrum of 22

$608^{\circ} 0$

$\angle 89 \cdot 9<-$

000.22

$90 Z \cdot \angle L$

$5 t 8 \cdot 8 I I$

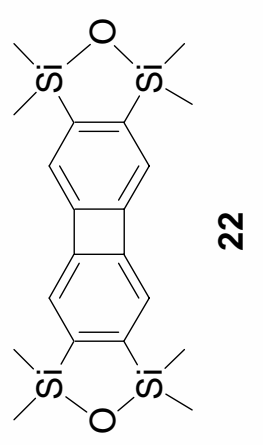

$389 \cdot 60 t$

$685^{\circ} 29 \mathrm{I}$

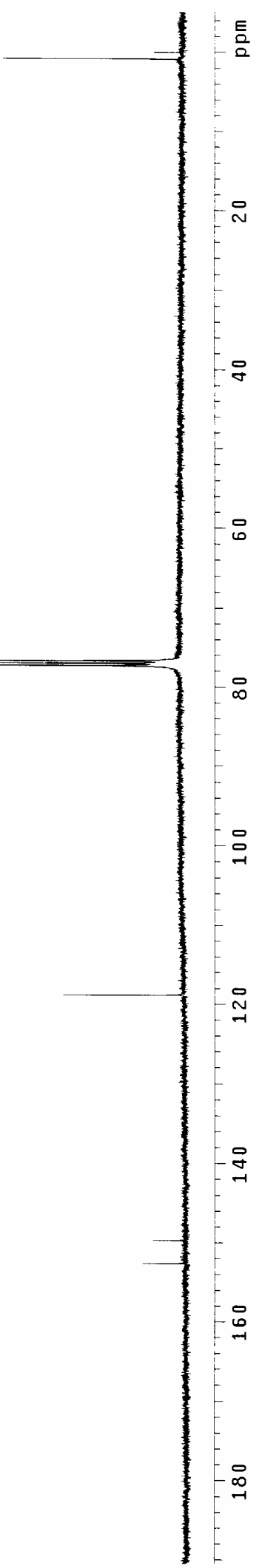


${ }^{1} \mathrm{H}$ NMR spectrum of $\mathbf{2 5 a}+\mathbf{2 6 a}$
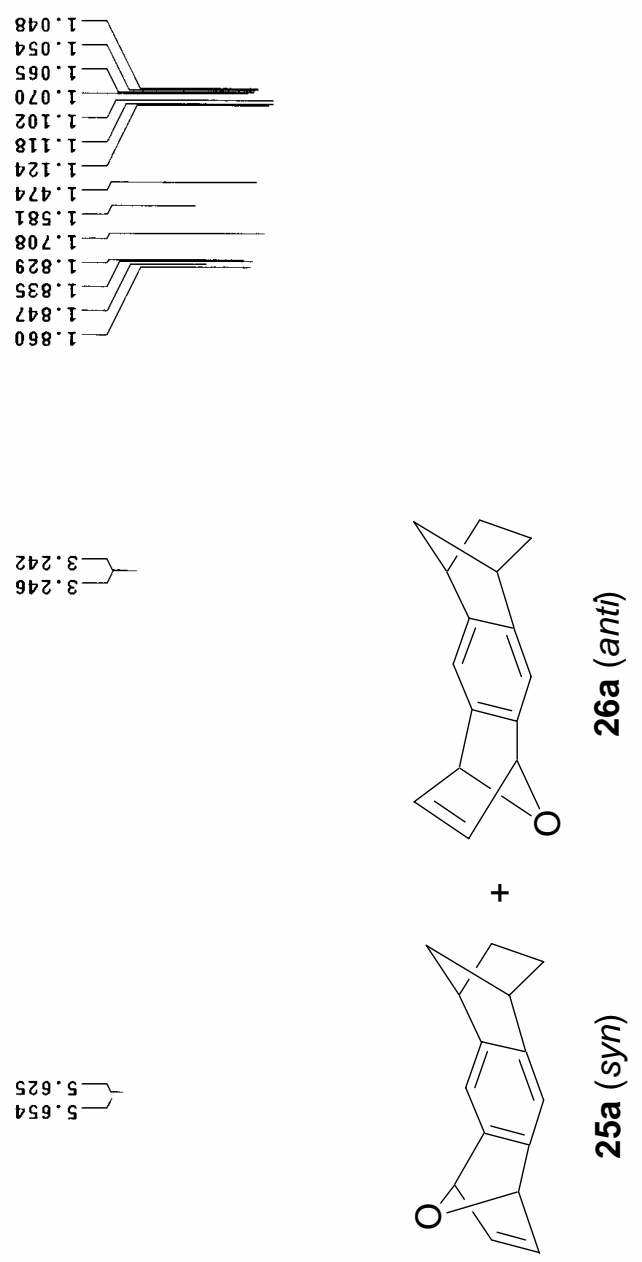

520.2

$\varepsilon<0^{\circ}<$

$860^{\circ} \mathrm{L}$

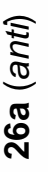

$=\stackrel{0}{\stackrel{2}{N}}$

है

ก็

$\stackrel{8}{i}$

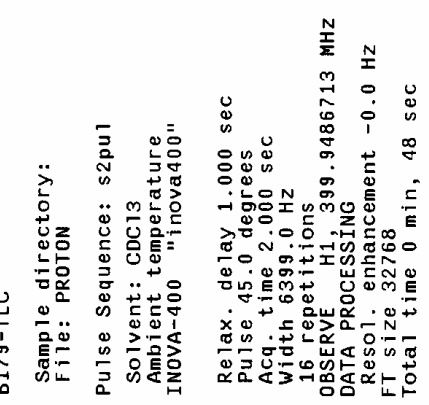


${ }^{13} \mathrm{C}$ NMR spectrum of $\mathbf{2 5 a}+\mathbf{2 6 a}$

$\angle \varepsilon 8 \cdot 92$

$8 z S^{\prime} \varepsilon$

$\varepsilon b S^{\circ} \varepsilon b$

$890.667=$

tI0.OS

$000^{\circ} \angle L$

$50 Z \angle L$

$98 \varepsilon \cdot 28$

$\varepsilon \mathrm{s} \cdot 28^{-}$

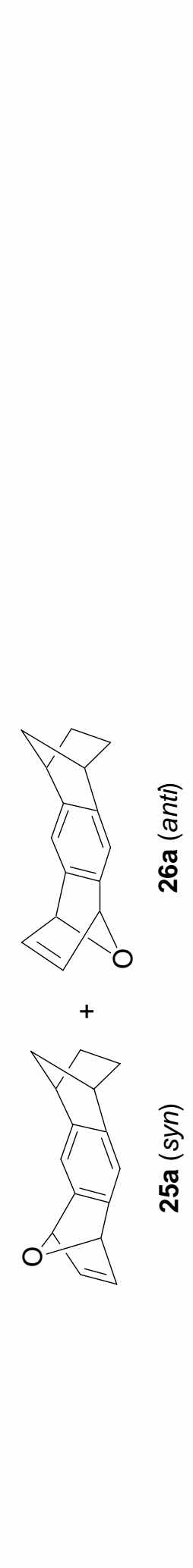

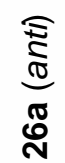

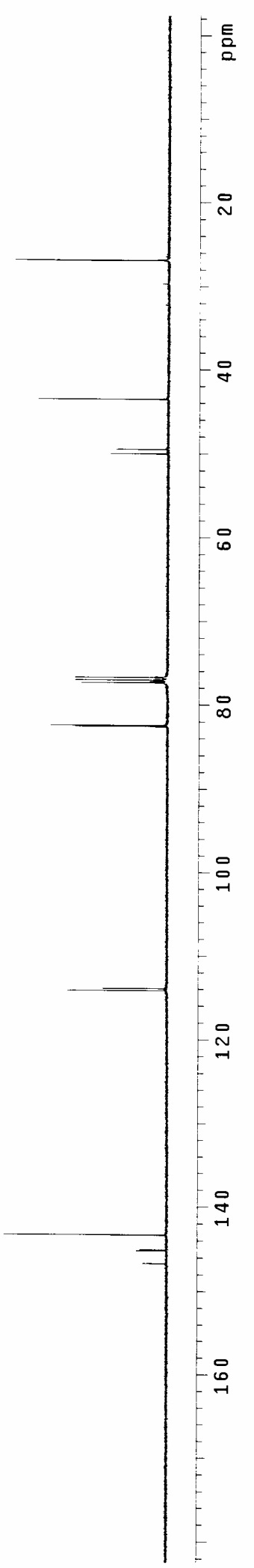

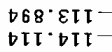

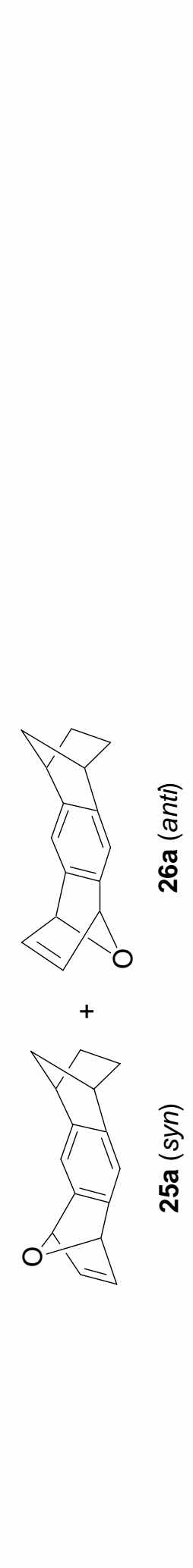

$\angle 0 \varepsilon \cdot \varepsilon b \tau$

$350.50 I$

EbI. $50 I$

$069^{\circ} 90 \mathrm{~T}$
$9 \varepsilon L^{\circ} 9 \mathrm{D} \tau$

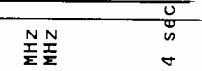

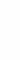

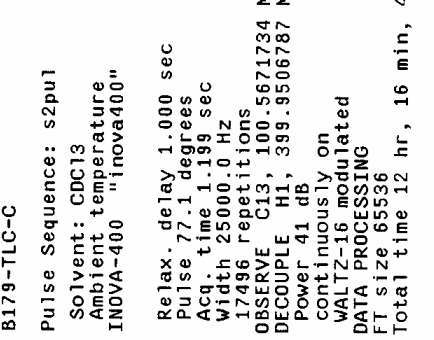


${ }^{1} \mathrm{H}$ NMR spectrum of $\mathbf{2 5 b}+\mathbf{2 6} \mathbf{b}$
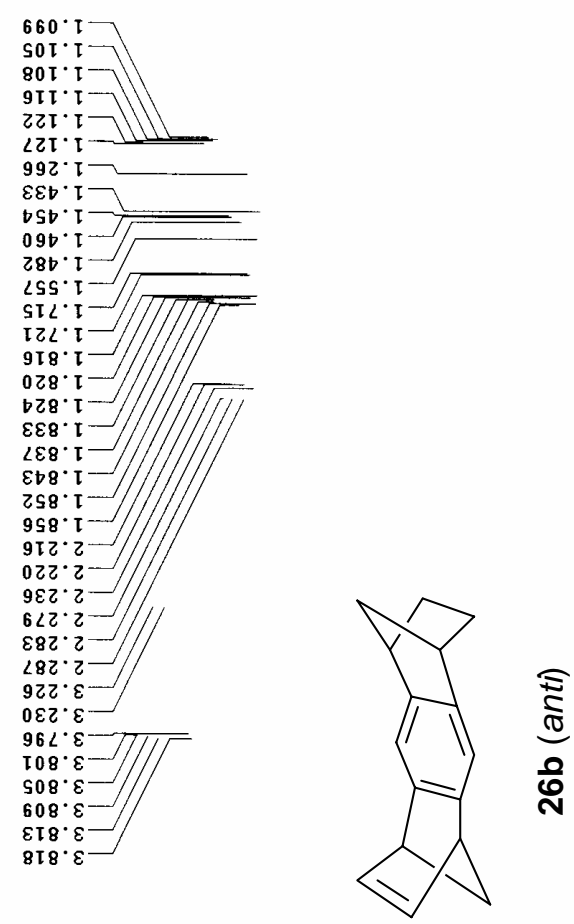

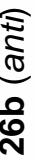

$\stackrel{n}{\sim}$

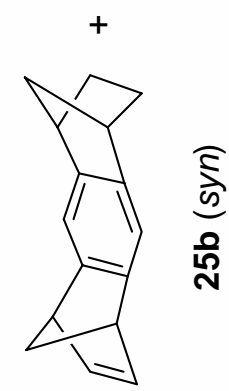

$082 \cdot 9$

$682 \cdot 9$

$682 \cdot 9$

$662 \cdot 9$

$960^{\circ} \cdot 2$

$092 \cdot 2$

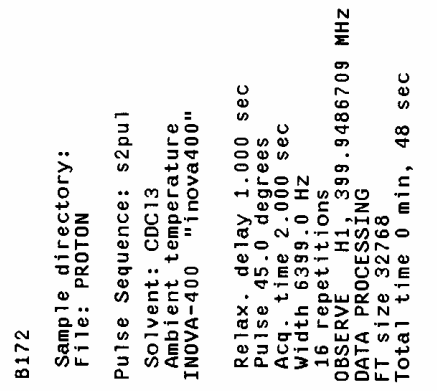

है
$\frac{0}{\text { है }}$ 
${ }^{13} \mathrm{C}$ NMR spectrum of $\mathbf{2 5 b}+\mathbf{2 6} \mathbf{b}$

$\varepsilon \varepsilon \tau \cdot<2]$

$\varepsilon<s^{\circ} \varepsilon \vee-$

$660 \cdot 60$

266.66

$\varepsilon \varepsilon \varepsilon^{\circ} 0 \mathrm{~s}-$

$S 50^{\circ} 0 L-$
$8 \angle 8 \cdot 0<$

$189 \cdot 92$

$600^{\circ}: \ll$

$986 \cdot \mathrm{ttI}$

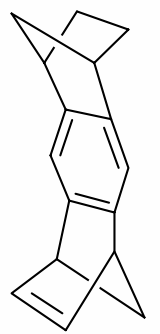

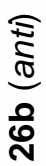

$\left\{\begin{array}{l}F E \\ F\end{array}\right.$
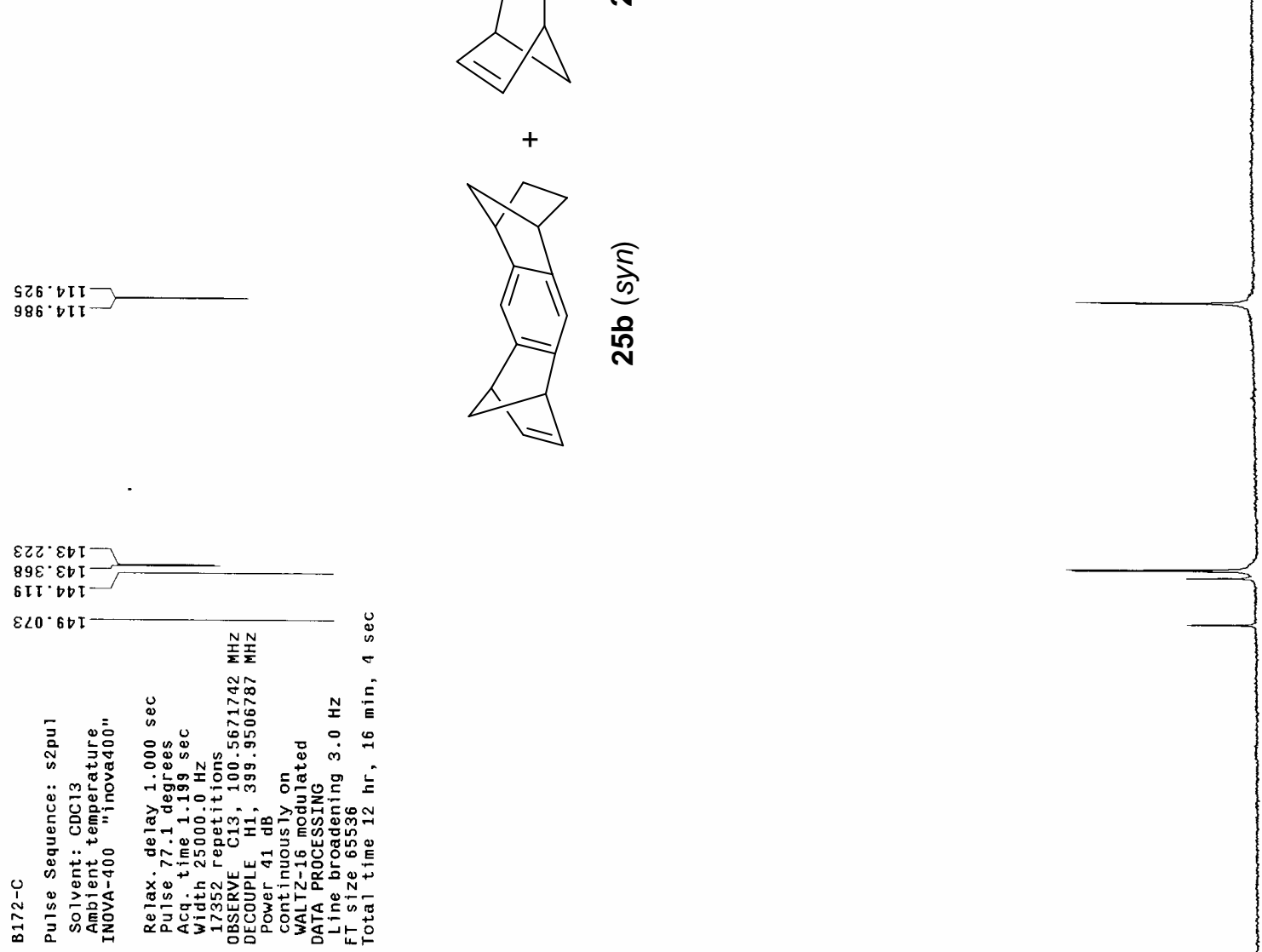
${ }^{1} \mathrm{H}$ NMR spectrum of 27
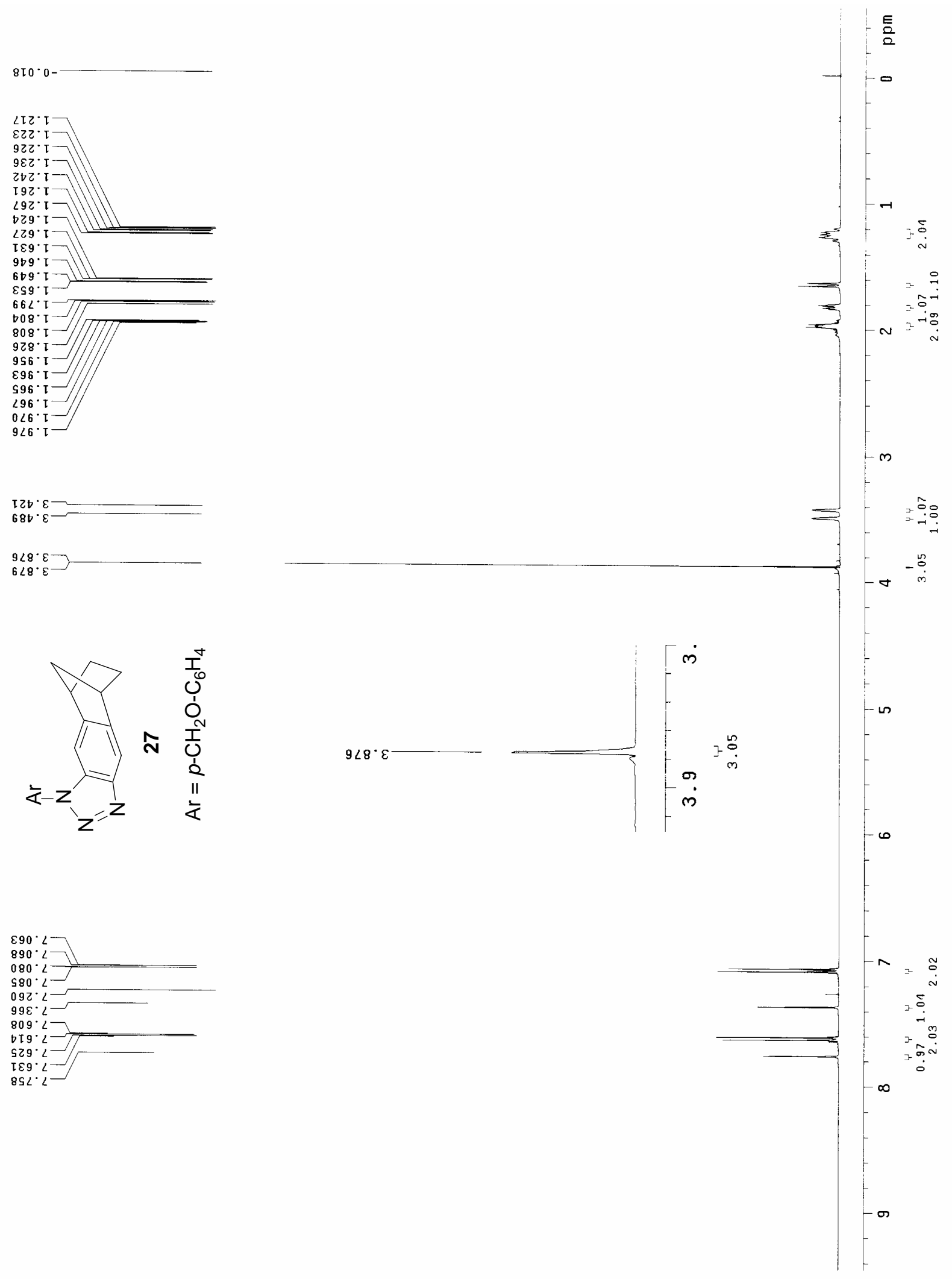

$\varepsilon 90^{\circ}\llcorner$

$890^{\circ} \mathrm{L}$

$580^{\circ} \mathrm{L}$

$092 \cdot L$

$99 \varepsilon \cdot<-$

Dโ $9^{\circ}<$

$529 \cdot 2$

โ $\varepsilon 9^{\circ}<-$

$8 \mathrm{~S} L^{\circ}\llcorner$

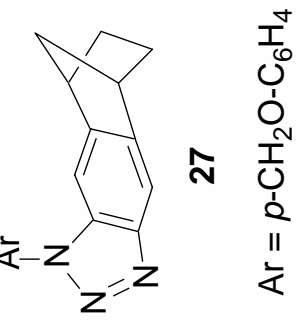

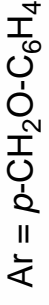


${ }^{13} \mathrm{C}$ NMR spectrum of 27

$9 \varepsilon z \cdot<z \square$
$s 9 \varepsilon \cdot<z-$

$96 I \cdot \varepsilon b-$

$829 \cdot 8 b$

sss.ss-
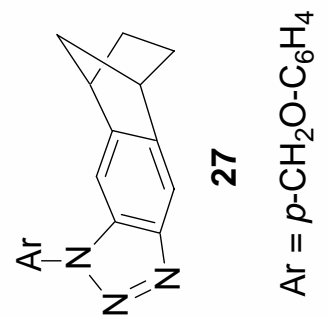

$089^{\circ} \cdot 92$

$000.2<$

$\varepsilon \mathrm{I} \varepsilon^{\circ}, 2 L$

$\angle Z 9^{\circ}$ โดI-

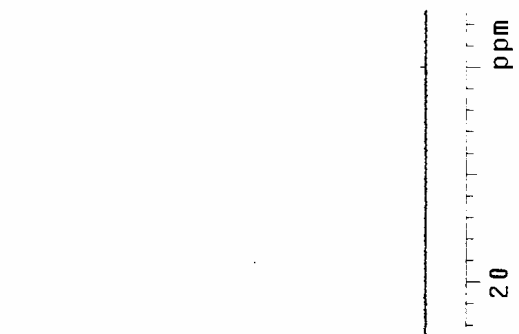

$\varepsilon 8 \mathrm{~s} \cdot 0 \mathrm{Tt}-$

$60 \angle \cdot D I T-$

$s \angle S^{\circ} b Z \tau-$

$\forall \angle T \cdot 0 E \mathrm{I}$

$D \supset 6 \cdot I \varepsilon I$

$D 6 \varepsilon^{\circ} \cdot S b T$
$8 z 0^{\circ} \cdot 9 b \tau$

LOV.0SI — _ _

$6 \varepsilon S^{\cdot} \cdot 6 S T^{-}$

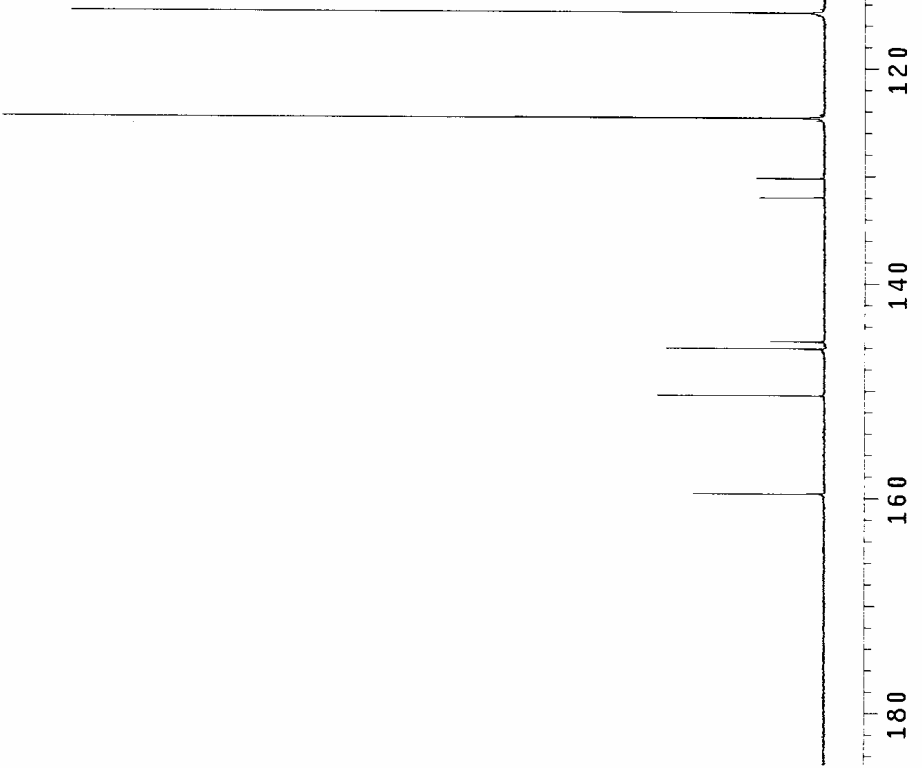


Ablert W. M. Lee et al. - Supporting Information

${ }^{1} \mathrm{H}$ NMR spectrum of 28

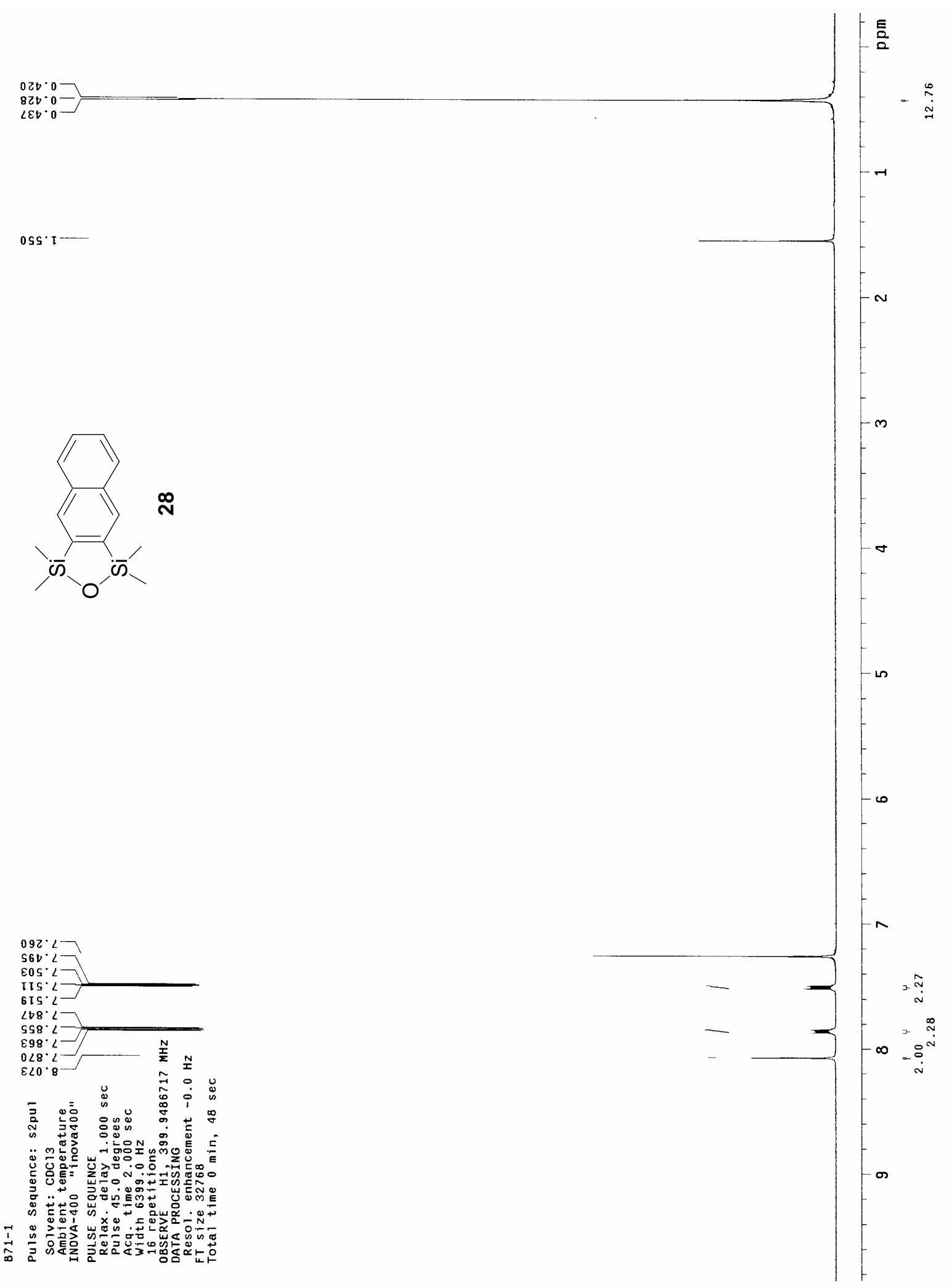


Ablert W. M. Lee et al. - Supporting Information

${ }^{13} \mathrm{C}$ NMR spectrum of $\mathbf{2 8}$

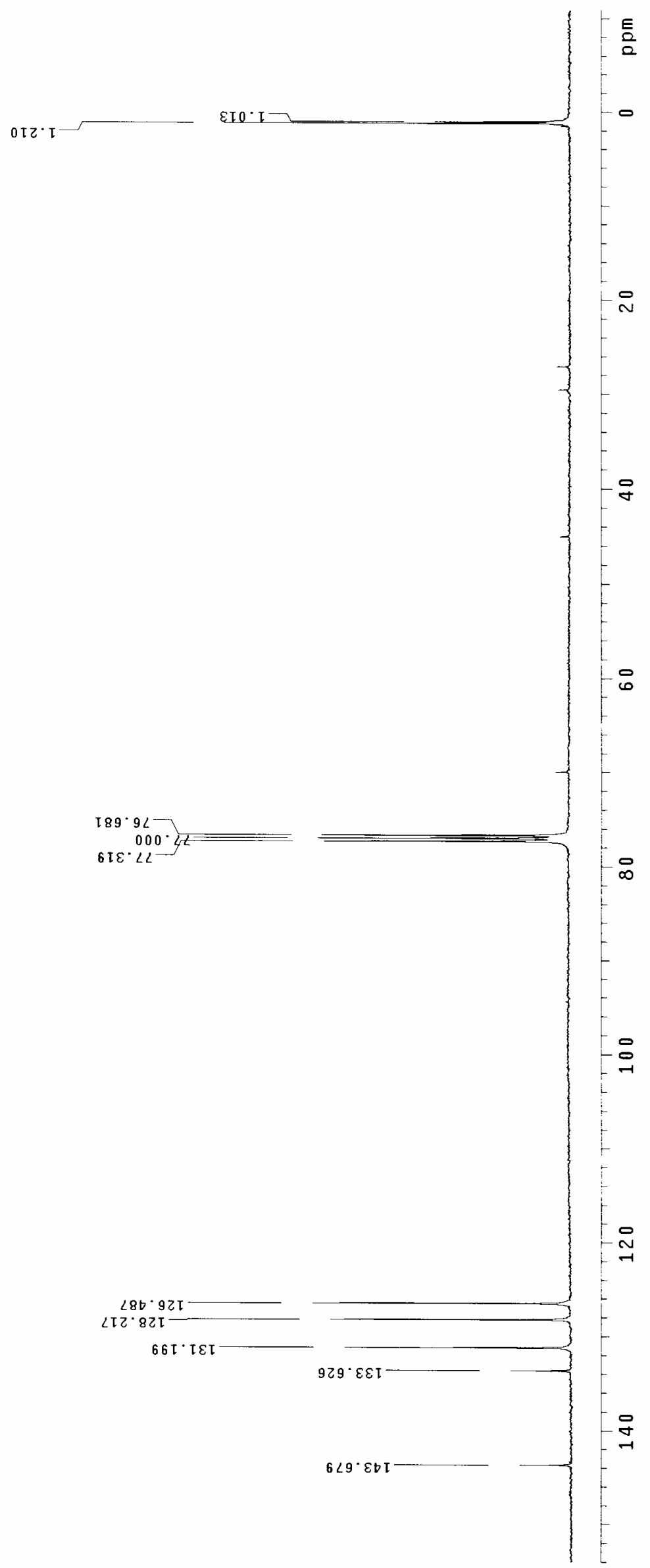


Ablert W. M. Lee et al. - Supporting Information

${ }^{1} \mathrm{H}$ NMR spectrum of $\mathbf{3 1}$

$620 \cdot 0$

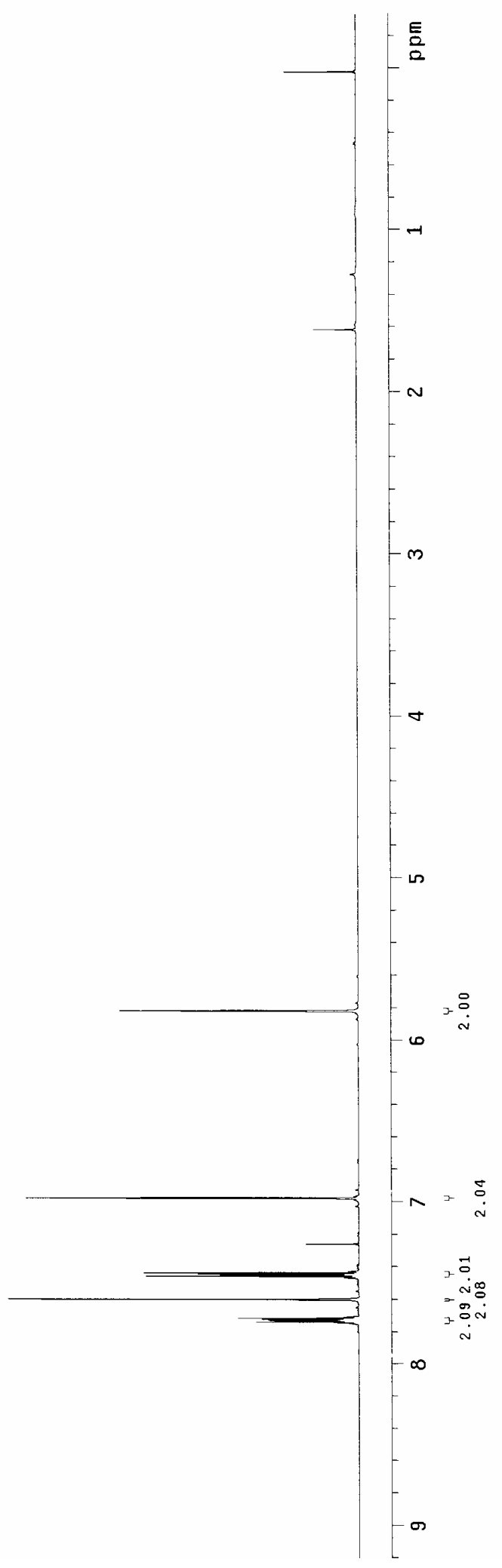

$818^{\circ}$.

028.2

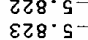

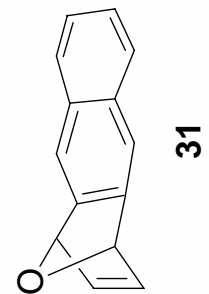

ले

$9 \angle 6$
$8 \angle 6 \cdot 9$

$186 \cdot 9$

$092 \cdot 2$

sto 2

ZSt $\angle$

$090^{\circ}<$

$009^{\circ} \mathrm{L}$

$109^{\circ} 2$

$9 \mathrm{I} L \cdot 2$

$92 L \angle$

$\varepsilon \varepsilon<\cdot<$

It $2 \cdot 2$ 
Ablert W. M. Lee et al. - Supporting Information

${ }^{13} \mathrm{C}$ NMR spectrum of 31

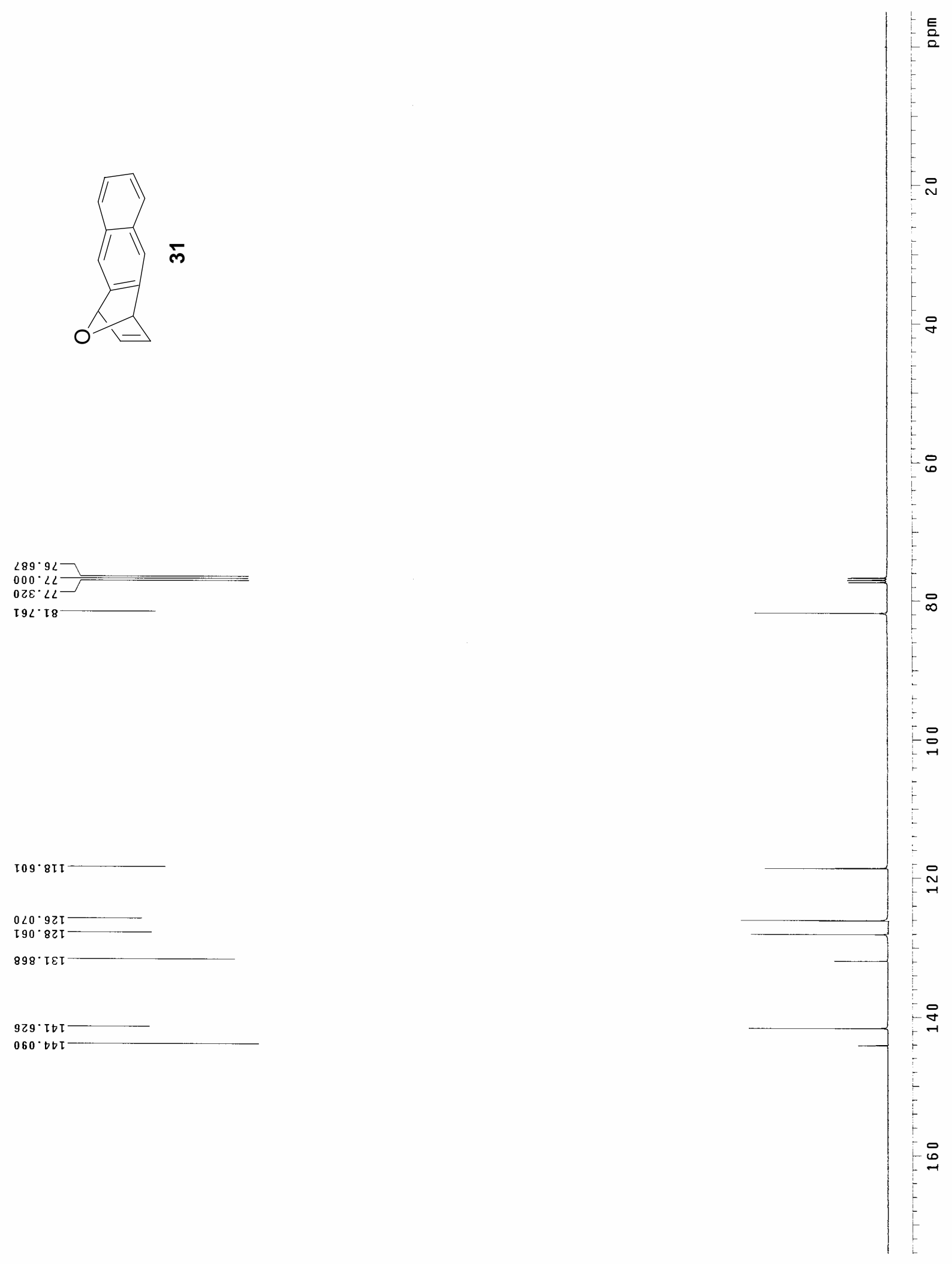


${ }^{1} \mathrm{H}$ NMR spectrum of 33a

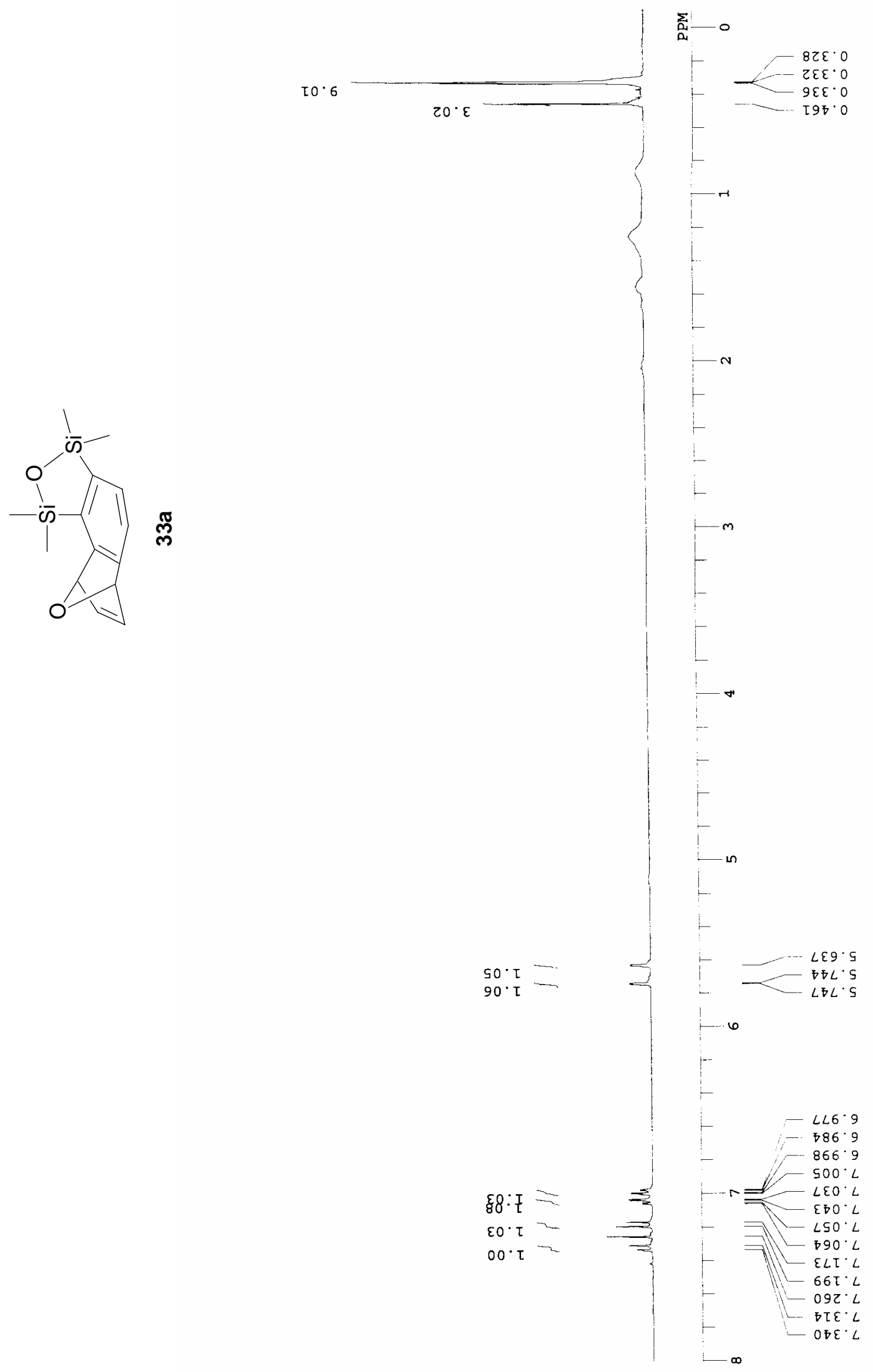


${ }^{13} \mathrm{C}$ NMR spectrum of 33a
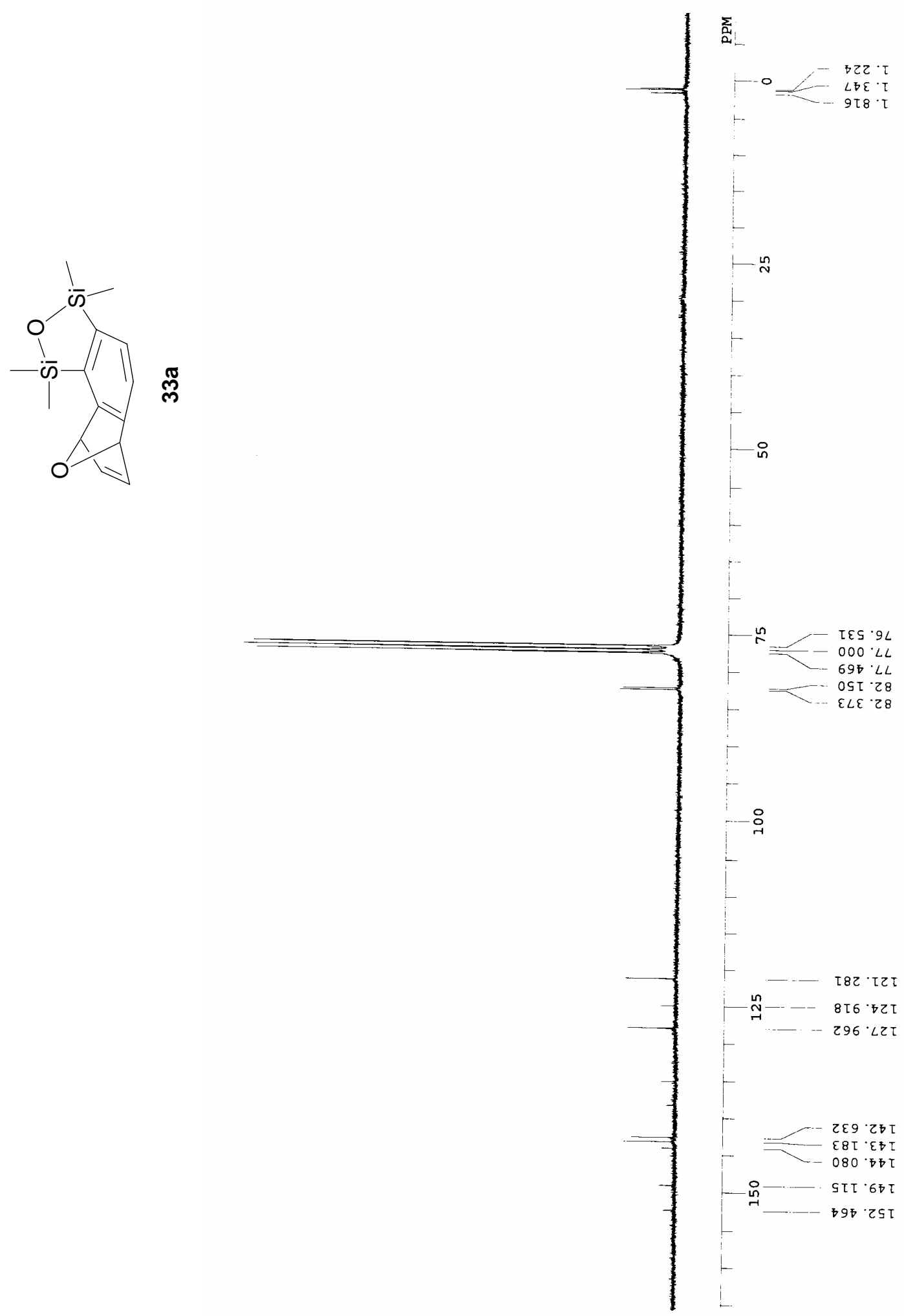
${ }^{1} \mathrm{H}$ NMR spectrum of $\mathbf{3 3 b}$

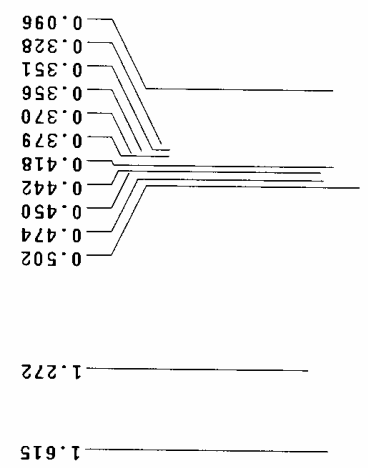

$682 \cdot z-$

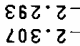

$\angle 0 \varepsilon \cdot 2$

$99 \varepsilon \cdot \tau$

$69 \varepsilon \cdot z-$

$6 \angle \varepsilon^{\circ} \cdot 2$

$\angle 8 \varepsilon \cdot 2$

$288^{\circ} \cdot 2-100$
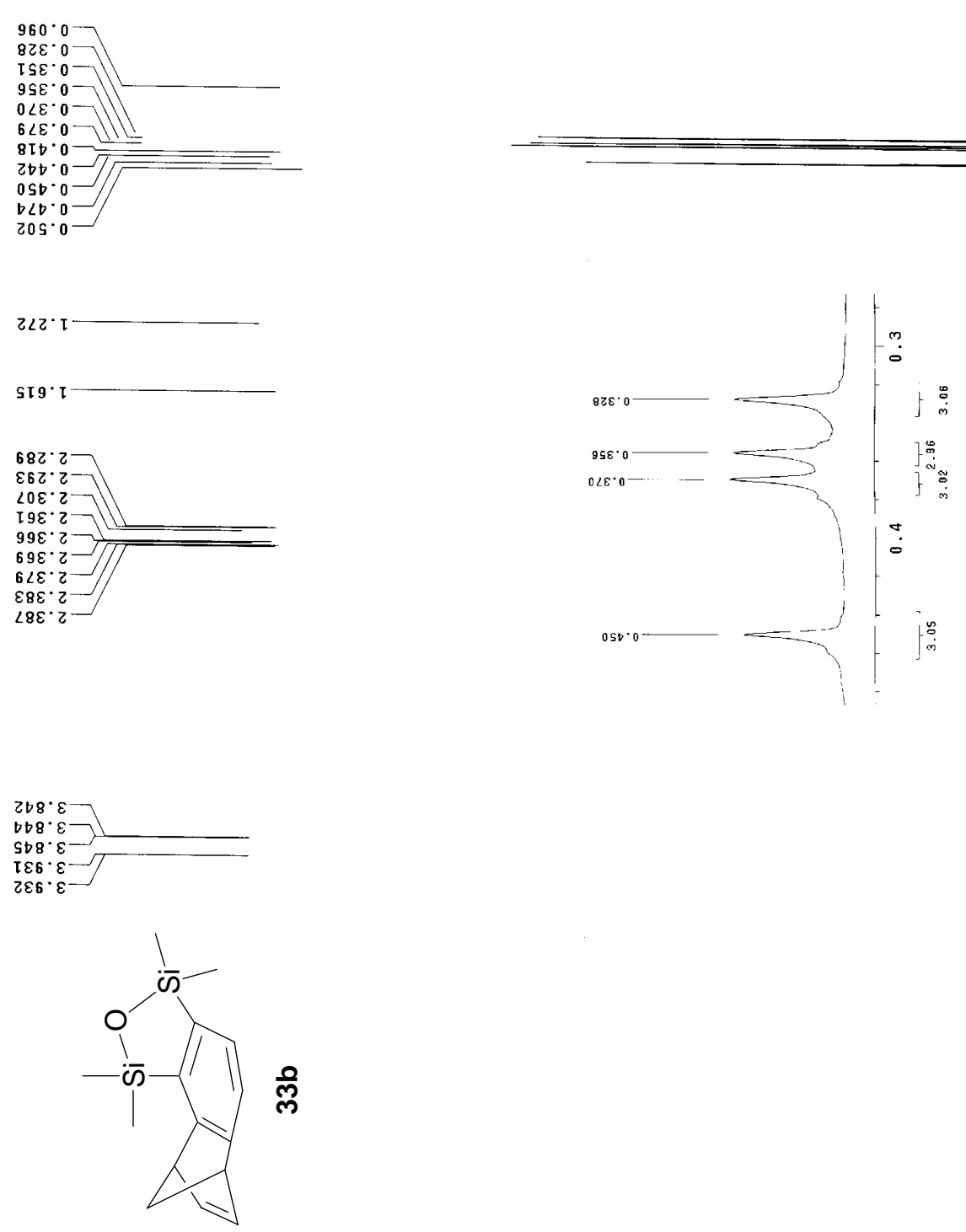

ले
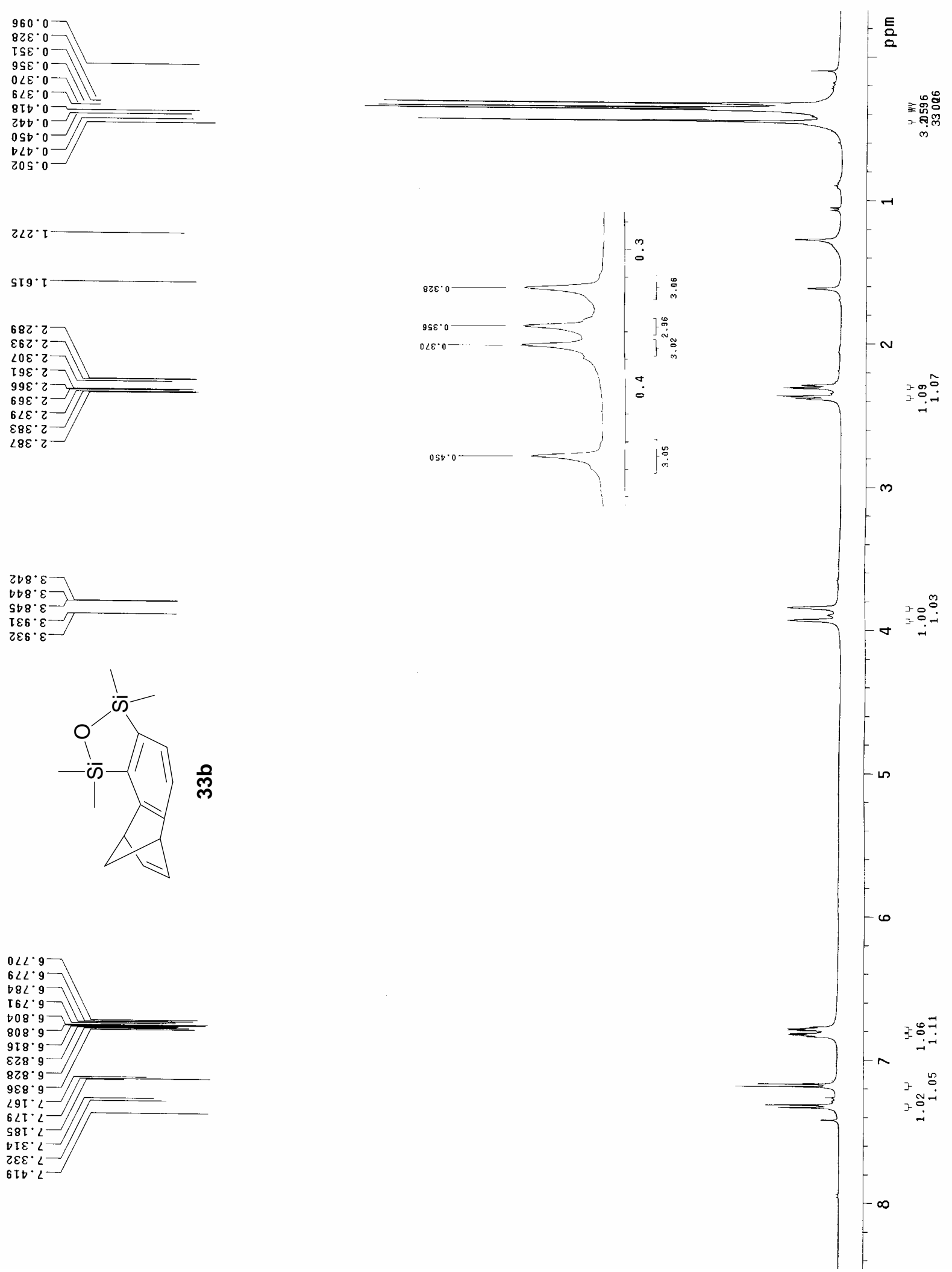
${ }^{13} \mathrm{C}$ NMR spectrum of $\mathbf{3 3 b}$

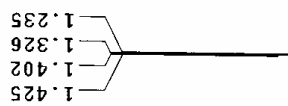

$890^{\circ} 0 \mathrm{~S}^{-}$

$665^{\circ} 0 S^{-}$

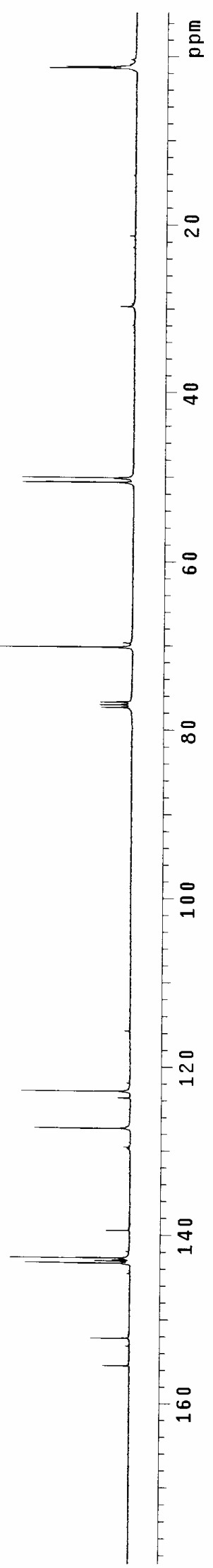

I St. $6 \varepsilon \mathrm{I}^{-}$

$90 L^{\circ} Z b I$

$0 \angle 0^{\circ} \varepsilon b I$

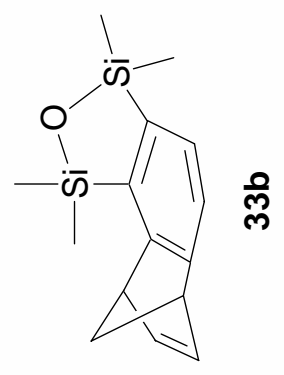

ले

$068 \cdot 2 z 1-$

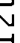

$\varepsilon \tau \varepsilon^{\circ} \cdot\llcorner Z \tau$

$6 I Z \cdot 2 S I$

$96 \mathrm{~b} \cdot \mathrm{sI}$ 
Ablert W. M. Lee et al. - Supporting Information

${ }^{1} \mathrm{H}$ NMR spectrum of 33c
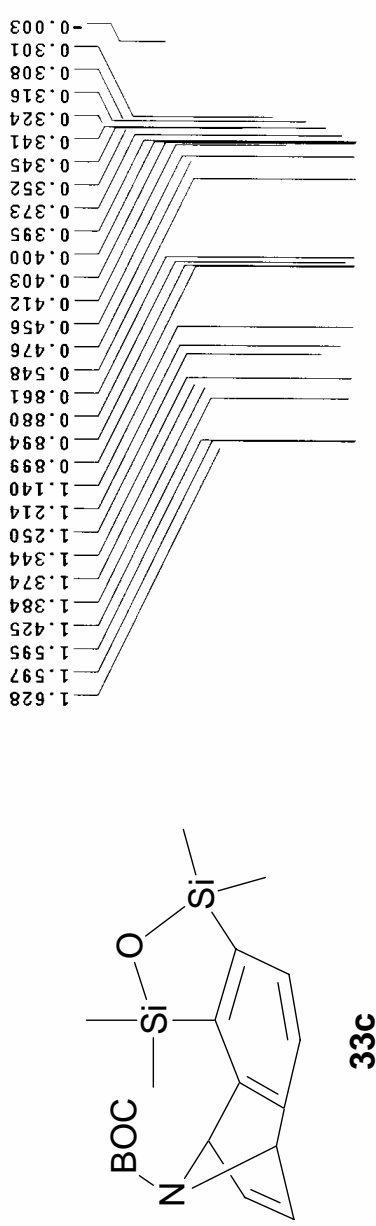

608.5

$8 \mathrm{st} \cdot \mathrm{s} \cdot \mathrm{s}$
$8 \mathrm{~L}$

ن্ল

0 โ 6.9

$S \varepsilon 6 \cdot 9$

956.9
$b 56.9$

$656 \cdot 9$
656

$\angle 56 \cdot 9$

$6<6 \cdot 9$

$966 \cdot 9$

S9I. 2

$09 z \cdot<$ -

(5)

$862 \cdot 2$

S०E $\cdot 2$

$\operatorname{si\varepsilon } \cdot<$

D $2 E \cdot<$

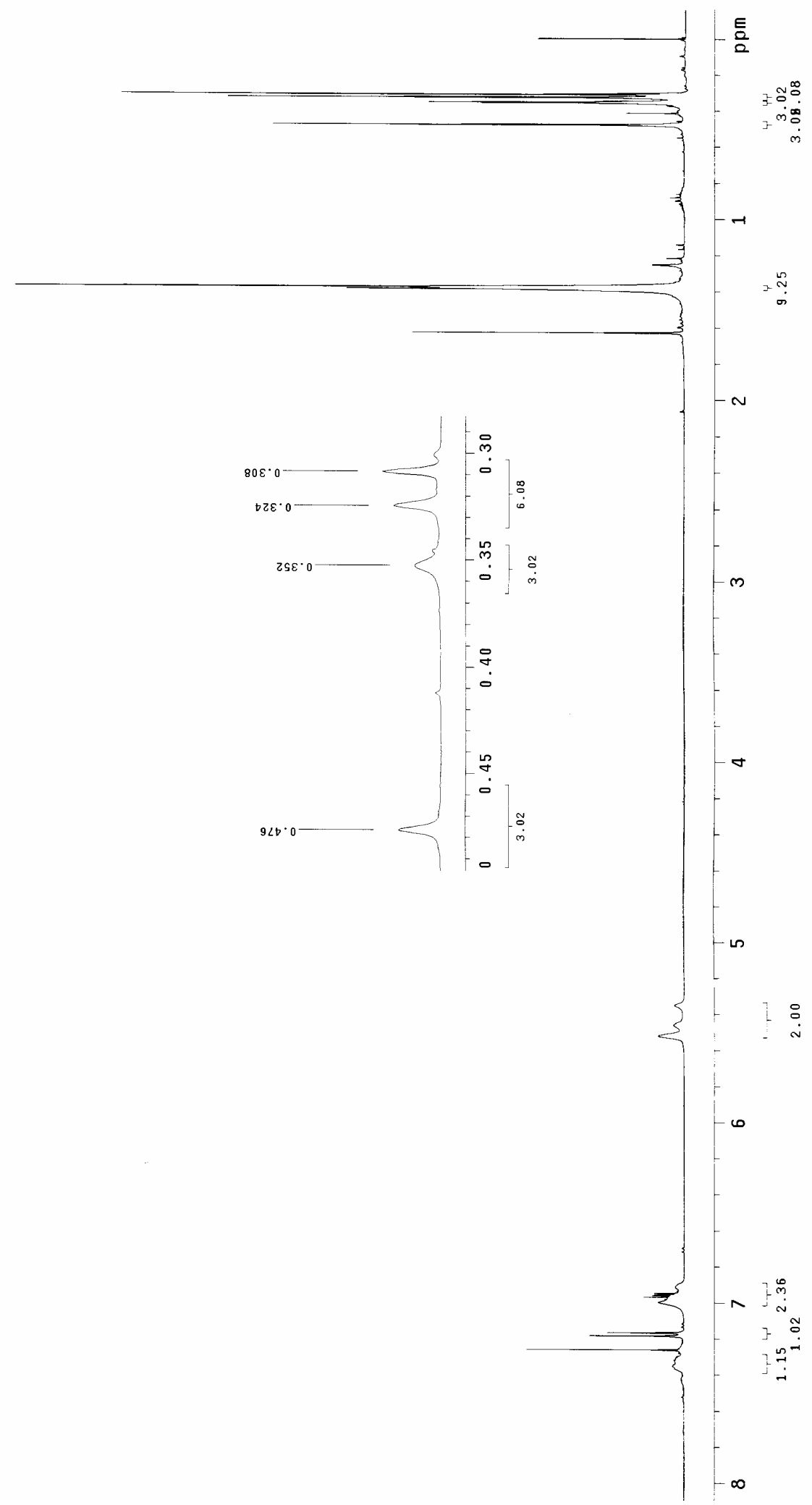


${ }^{13} \mathrm{C}$ NMR spectrum of 33c

ESO.I-

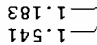

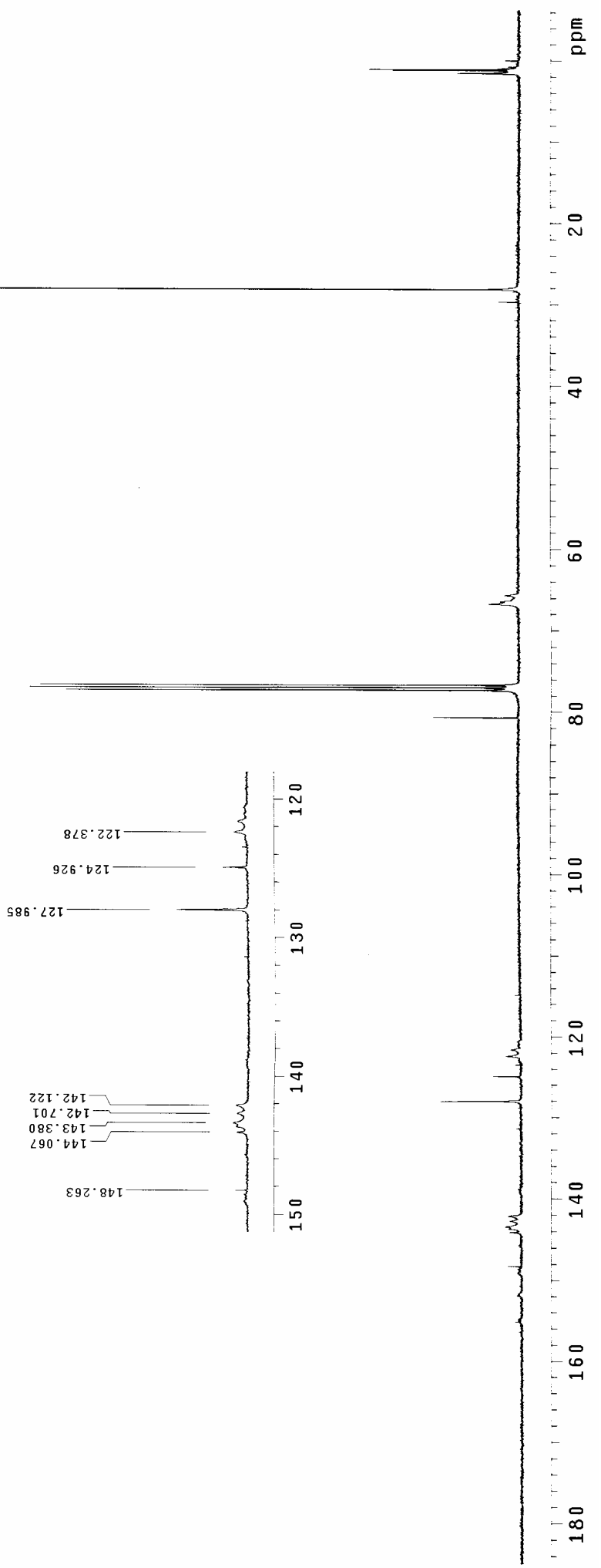

$089 \cdot 9<-$

$000^{\circ} \angle L$

$02 \varepsilon \cdot 22$

$926 \cdot 62 \mathrm{I}$

$586^{\circ} \angle Z I$

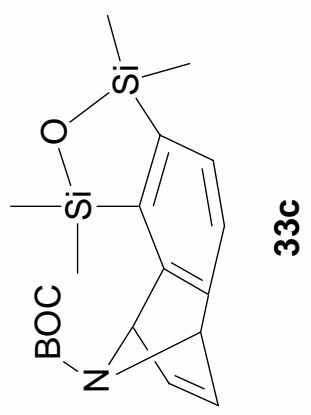

틀

$\infty$

i

$\stackrel{-0}{0}$

$\infty$ 
${ }^{1} \mathrm{H}$ NMR spectrum of $\mathbf{3 4 b}$
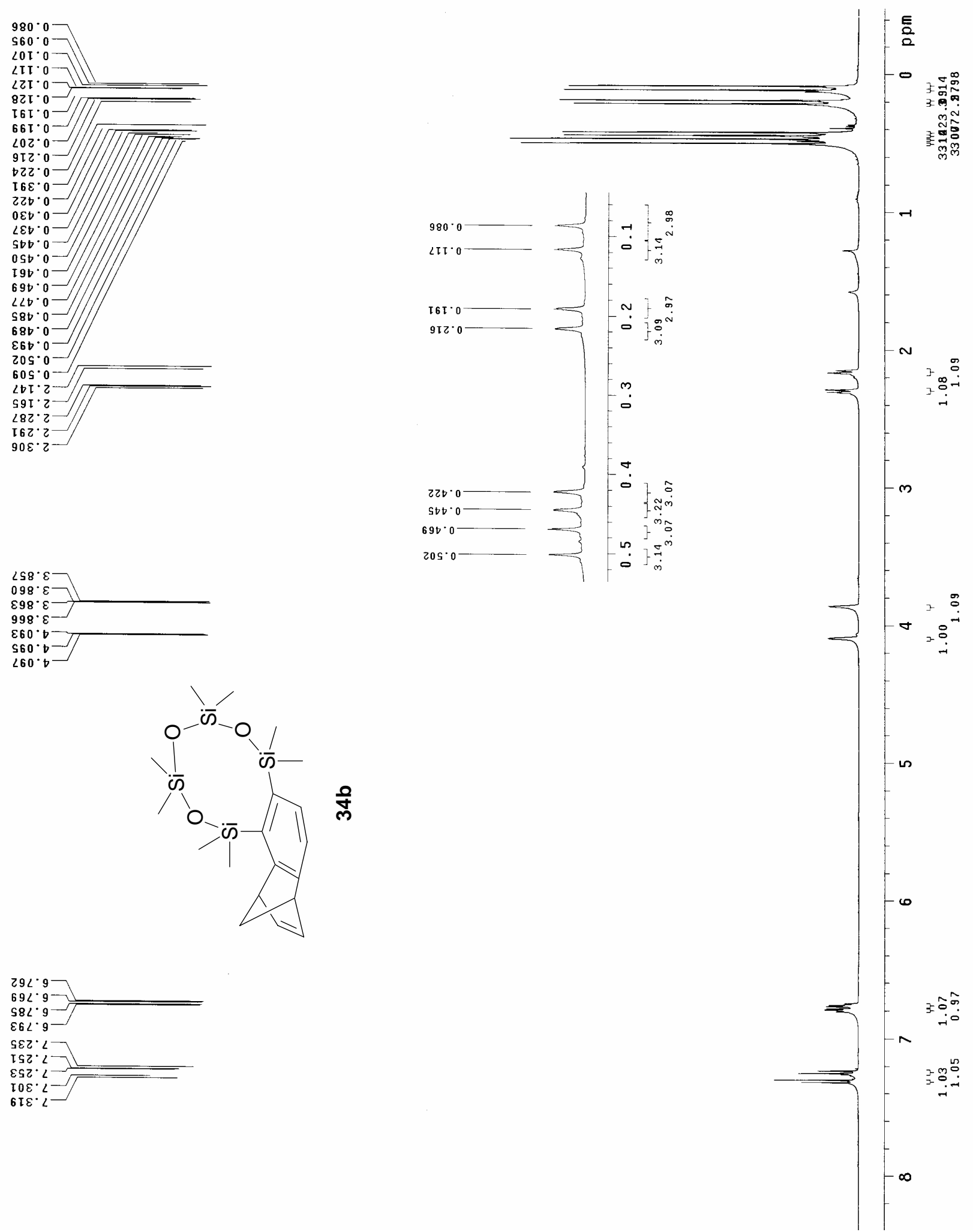

$\angle S 8^{\circ} \cdot$
$098^{\circ} \cdot$

$\varepsilon 98^{\circ} \varepsilon$

$\left(988^{-} \varepsilon\right.$

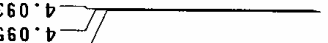

$\angle 60^{\circ} \circ$

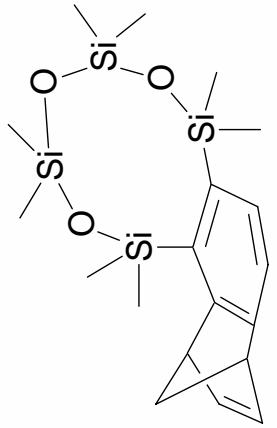

욤

$29 \angle \cdot 9-$

$69<\cdot 9$

$\varepsilon 8<\cdot 9$

$s \varepsilon z \cdot L$

I $52 \cdot 2$

$\varepsilon 52 \cdot 2$

$0 \varepsilon \cdot 2-$ 
${ }^{13} \mathrm{C}$ NMR spectrum of $\mathbf{3 4 b}$

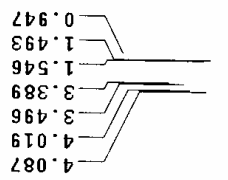

$882 \cdot 6 b-$

$\varepsilon \angle \varepsilon^{\circ}$ Is -

$660^{\circ} 89^{-}$

I $89 \cdot 9<$

$000^{\circ} \angle L$

G I $\angle L$

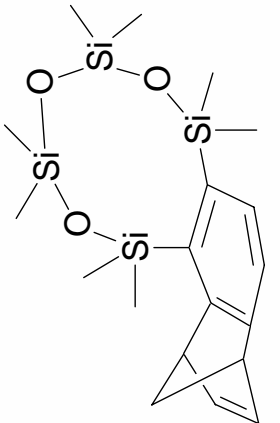

से

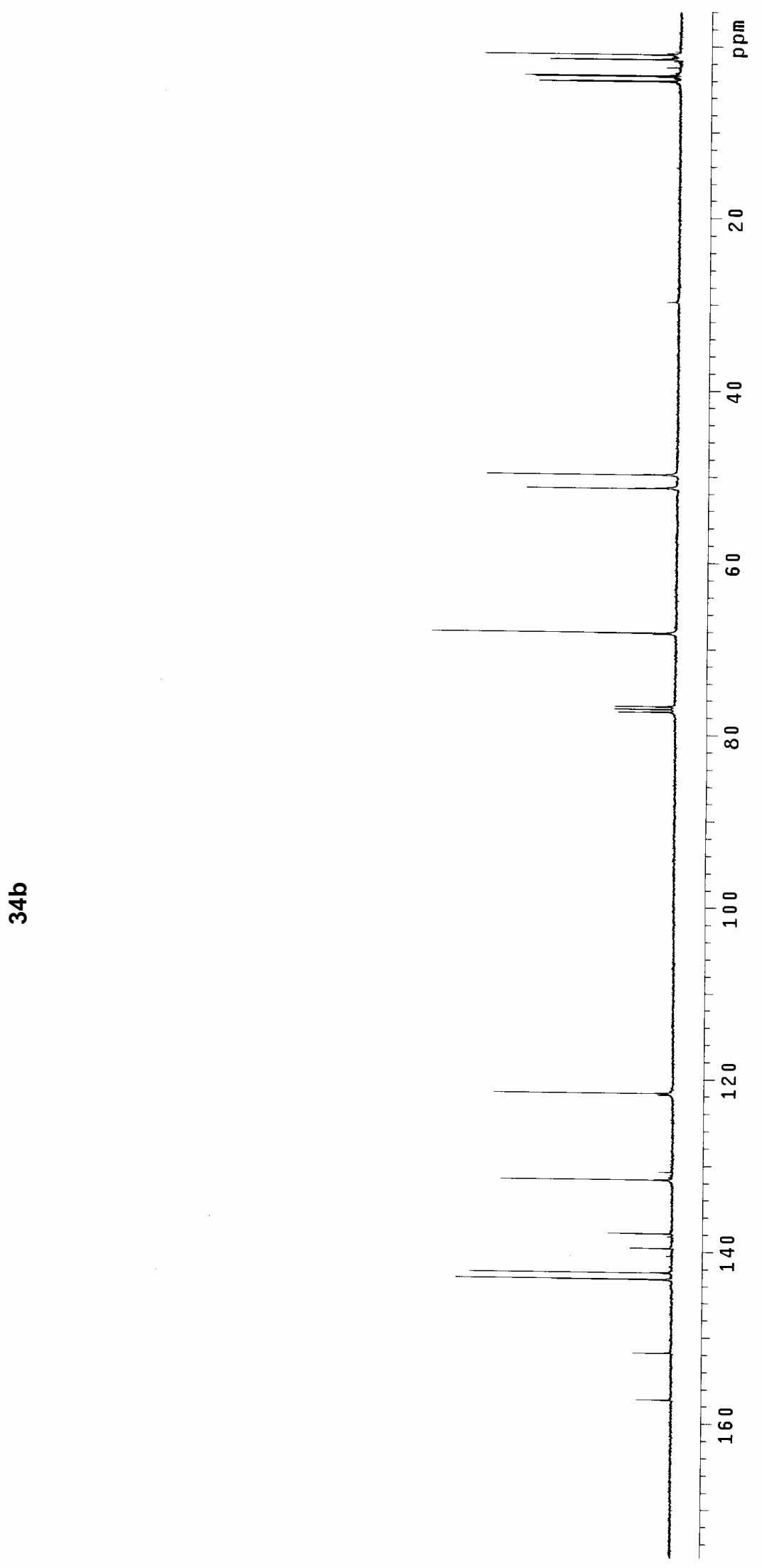

$S 98^{\circ} \angle \varepsilon \mathrm{I}$

$025 \cdot 6 \varepsilon 1-$

$89 \tau \cdot \varepsilon \mathrm{LI}$

$6 L L$ ISI — _ _ _ _

IVI $\angle S T+\ldots$ 
${ }^{1} \mathrm{H}$ NMR of $35 b$
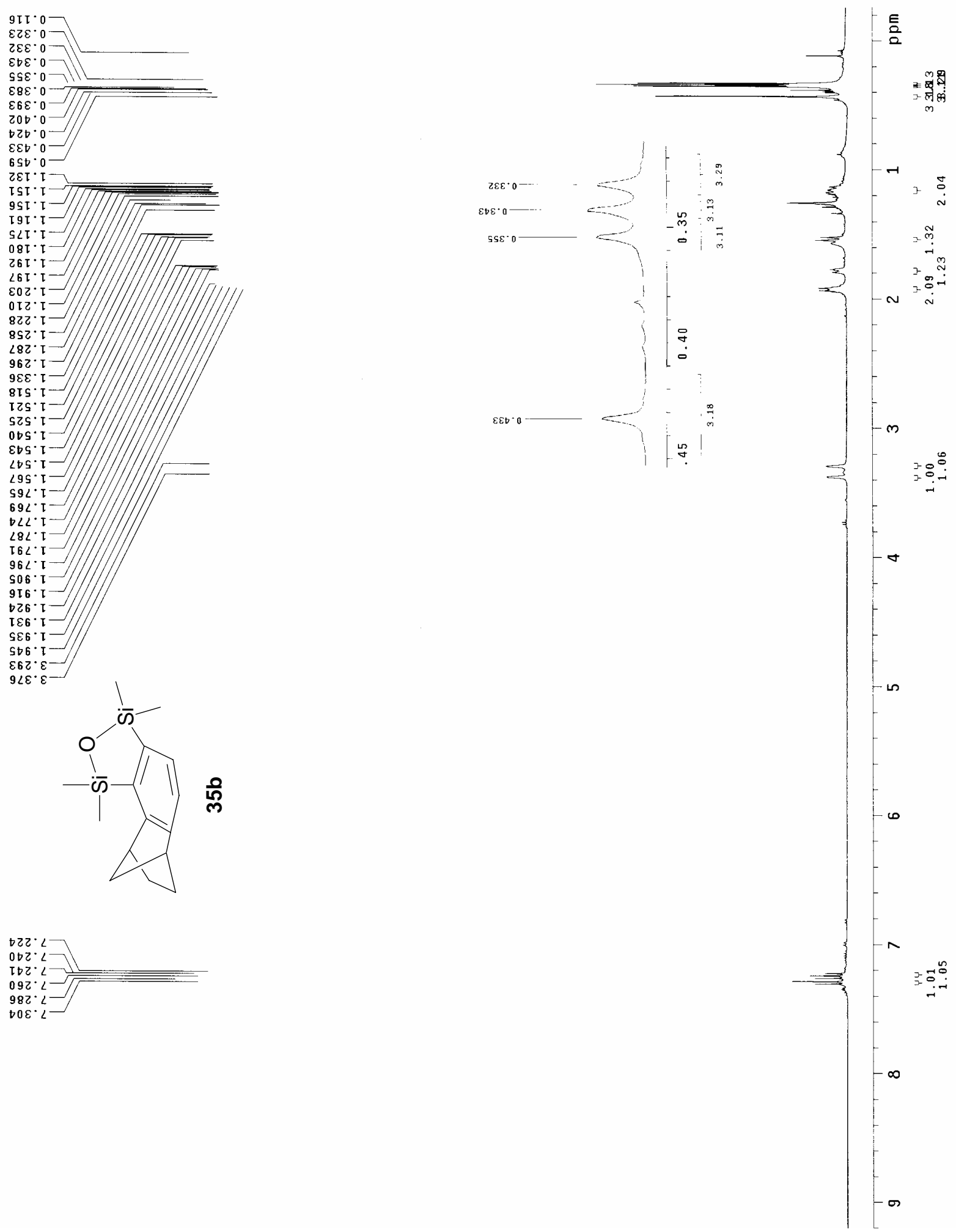
${ }^{13} \mathrm{C}$ NMR spectrum of $35 b$

$$
\begin{array}{ll}
\angle G I \cdot T \\
9 Z E \cdot I
\end{array}
$$

$9 Z \varepsilon^{\prime} \mathrm{I}$

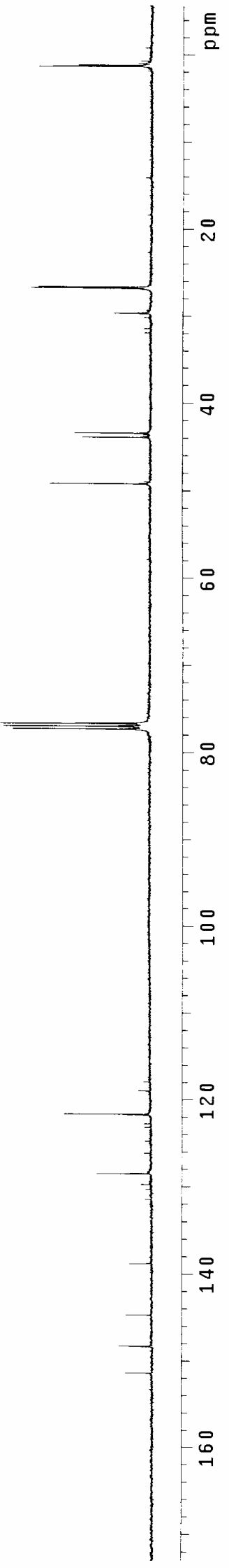

$\checkmark 99 \cdot 9 z$

$108 \cdot 9 z$

$80 b^{\circ} \varepsilon$

$8 \mathrm{~T} \cdot$

$189 \cdot 9<$

$000^{\circ} \angle 2 \div$

$6 โ \varepsilon^{\circ} \ll L$

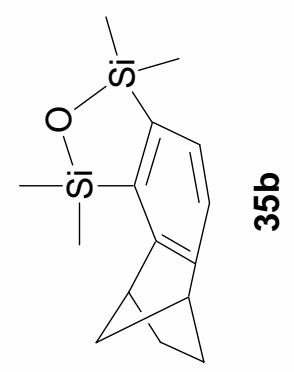

$9 \angle g^{\circ} \operatorname{TLI-~}$

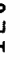

$960 \cdot 82 I$

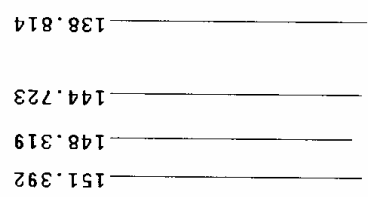

ํํำ 
Ablert W. M. Lee et al. - Supporting Information

${ }^{1} \mathrm{H}$ NMR spectrum of $35 \mathrm{c}$

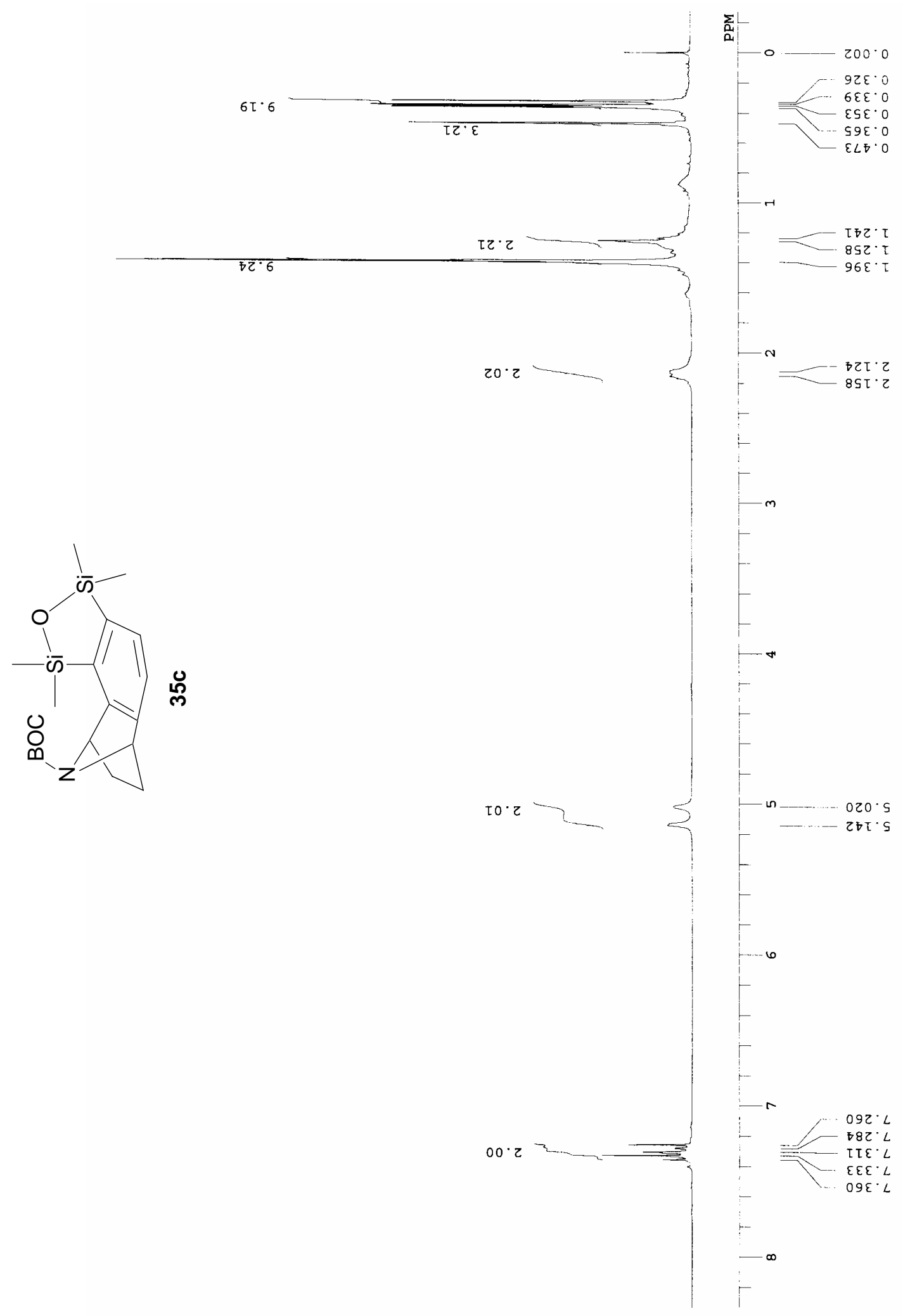


Ablert W. M. Lee et al. - Supporting Information

${ }^{13} \mathrm{C}$ NMR spectrum of $35 \mathrm{c}$

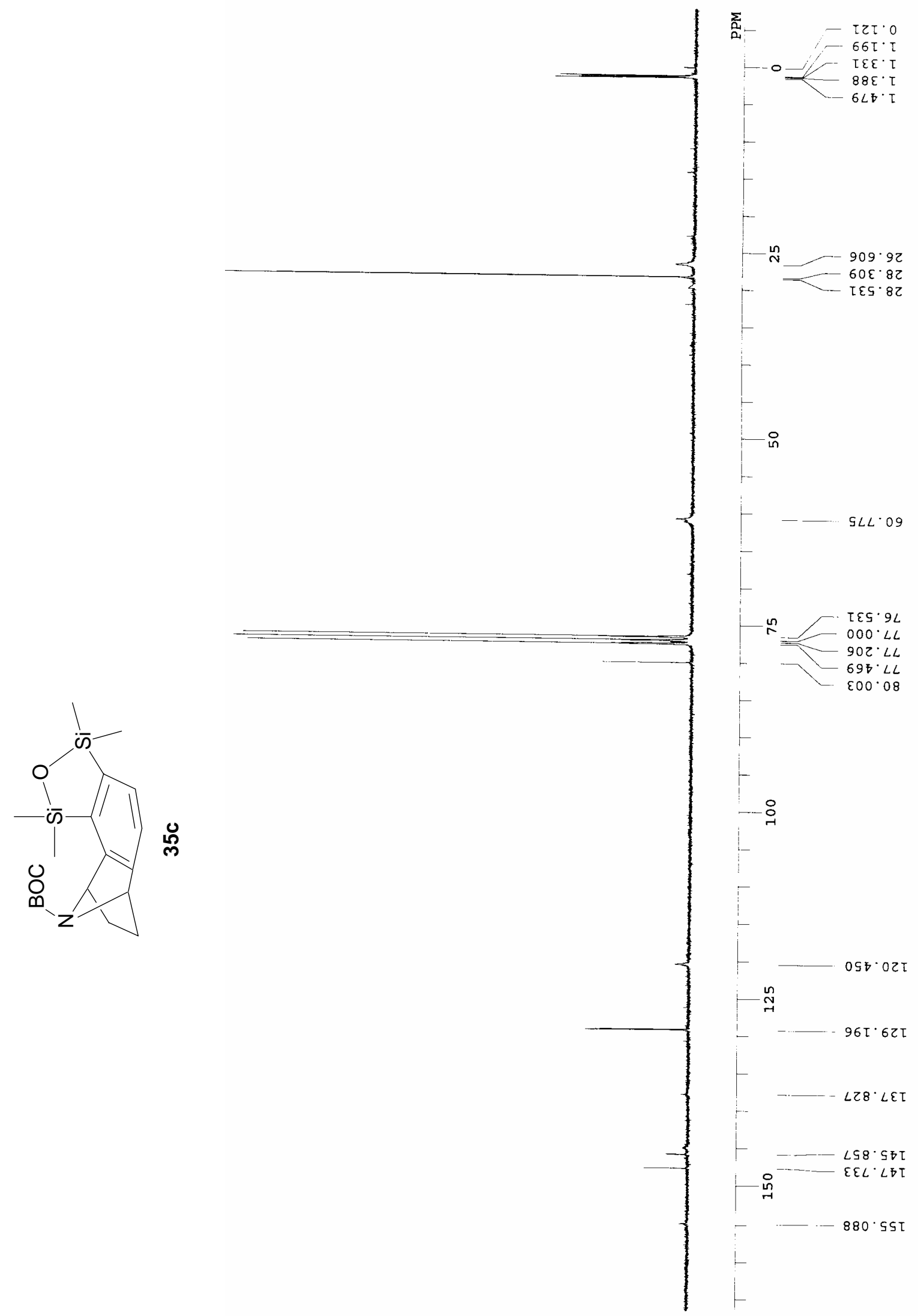




\section{${ }^{1} \mathrm{H}$ NMR spectrum of $\mathbf{3 7} \mathbf{b}+\mathbf{3 8 b}$}
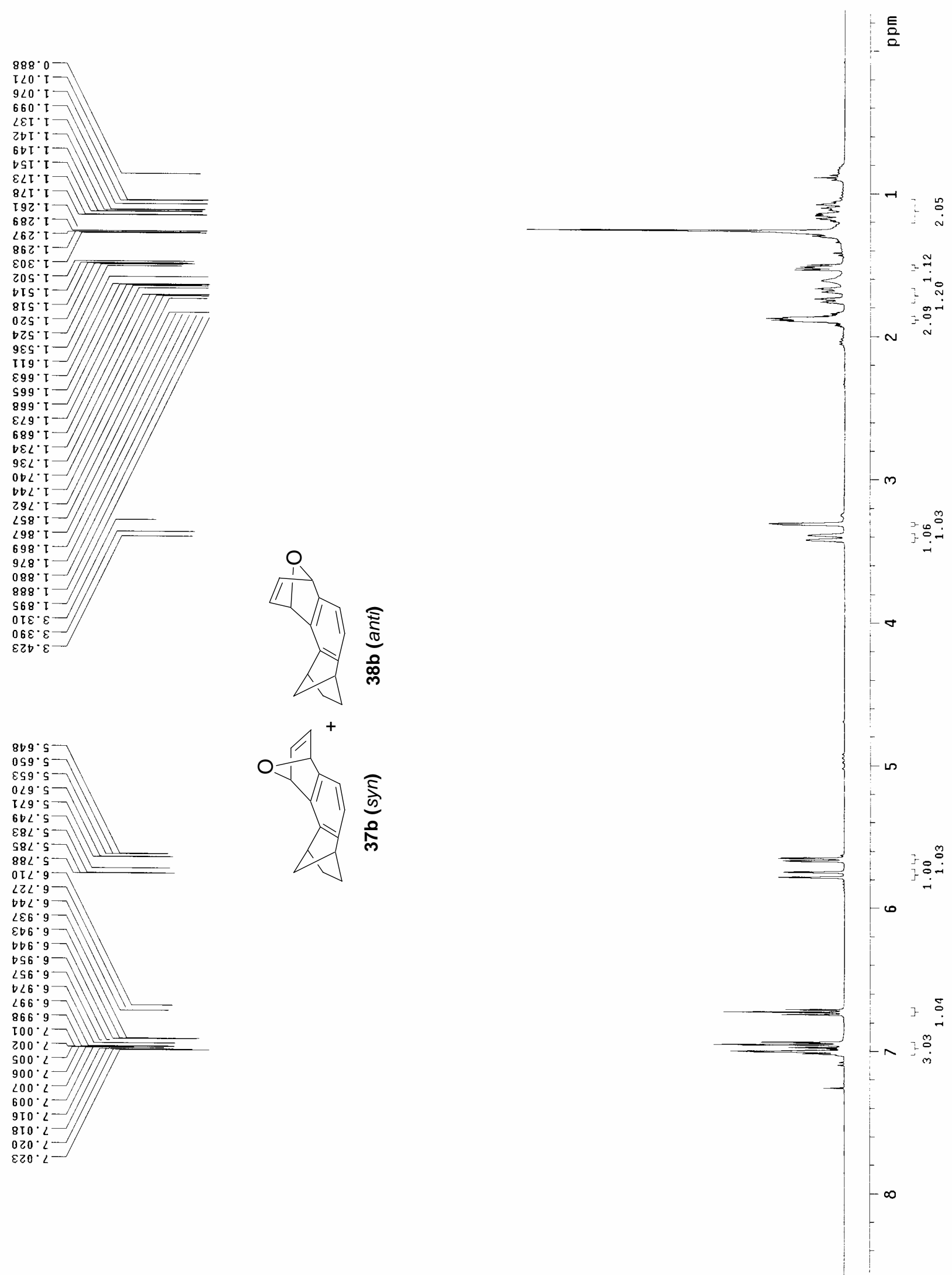
${ }^{13} \mathrm{C}$ NMR spectrum of $\mathbf{3 7 b}+\mathbf{3 8 b}$

$988^{\circ} \cdot 9 z$

$591 \cdot \angle \mathrm{Z}$

$591 \cdot 28$

$\angle \angle I \cdot \tau t$

SOb. It

$s Z 2 \cdot \varepsilon b$

$\varepsilon 62 \cdot 8 t$

$0<8^{\circ} 80$

$189 \cdot 9<$

$000^{\circ} 22$

$S Z L \cdot 08$

$28 \mathrm{I} \cdot 28$

$905 \cdot 28$

$708^{\circ}$ SII

886.911
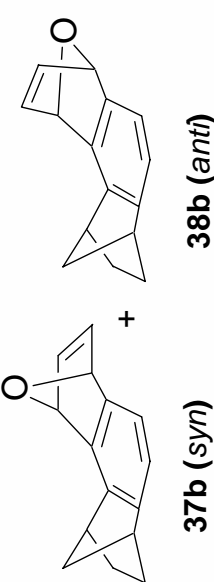

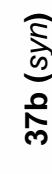

$820.0 \mathrm{TI}$

$9 Z \varepsilon \cdot 2 \circ \tau$

$2 \angle 8^{\circ} \mathrm{ZDI}$

$66 I^{\circ} \varepsilon D I$
$\angle S 9 \cdot S D I$

$896 \cdot S t I$

$866 \cdot S t I$ 